\title{
Geometric measures of quantum correlations: characterization, quantification, and comparison by distances and operations
}

\author{
W. Roga ${ }^{1,2 *}$, D. Spehner ${ }^{3,4 \dagger}$, and F. Illuminati ${ }^{1,5 \ddagger}$ \\ 1 Dipartimento di Ingegneria Industriale, Università degli Studi di Salerno, \\ Via Giovanni Paolo II 132, I-84084 Fisciano (SA), Italy \\ 2 Department of Physics, John Anderson Building, \\ University of Strathclydeb, 107 Rottenrow, Glasgow, G4 ONG, UK \\ 3 Univ. Grenoble Alpes and CNRS, Institut Fourier, F-38000 Grenoble, France \\ ${ }^{4}$ CNRS and univ. Grenoble Alpes, LPMMC, F-38000 Grenoble, France and \\ 5 INFN, Sezione di Napoli, Gruppo collegato di Salerno, I-84084 Fisciano (SA), Italy
}

(Dated: February 6, 2016)

\begin{abstract}
We investigate and compare three distinguished geometric measures of bipartite quantum correlations that have been recently introduced in the literature: the geometric discord, the measurementinduced geometric discord, and the discord of response, each one defined according to three contractive distances on the set of quantum states, namely the trace, Bures, and Hellinger distances. We establish a set of exact algebraic relations and inequalities between the different measures. In particular, we show that the geometric discord and the discord of response based on the Hellinger distance are easy to compute analytically for all quantum states whenever the reference subsystem is a qubit. These two measures thus provide the first instance of discords that are simultaneously fully computable, reliable (since they satisfy all the basic Axioms that must be obeyed by a proper measure of quantum correlations), and operationally viable (in terms of state distinguishability). We apply the general mathematical structure to determine the closest classical-quantum state of a given state and the maximally quantum-correlated states at fixed global state purity according to the different distances, as well as a necessary condition for a channel to be quantumness breaking.
\end{abstract}

PACS numbers: 03.67.Mn, 03.65.Ud, 03.65.Ta

\section{INTRODUCTION}

The characterization and quantification of quantum correlations in composite quantum systems is of primary importance in quantum information theory. In particular, it is a prerequisite for understanding the origin of the quantum advantages in tasks of quantum technology and quantum information processing. It has been recognized in the last decade that quantum correlations may be present even in separable mixed states. The quantum-correlated states are singled out by a non-vanishing value of the entropic quantum discord (whose definition and properties will be recalled in the following) [1 [3]. States of a bipartite system $A B$ with vanishing quantum discord with respect to subsystem $A$ possess only classical correlations between $A$ and $B$ and are called classical-quantum (or $A$-classical) states. They are of the form

$$
\sigma_{\mathrm{cq}}=\sum_{i=1}^{n_{A}} q_{i}\left|\alpha_{i}\right\rangle\left\langle\alpha_{i}\right| \otimes \varrho_{B \mid i},
$$

where $\left\{\left|\alpha_{i}\right\rangle\right\}$ is an orthonormal basis of the Hilbert space $\mathcal{H}_{A}$ of the reference subsystem $A, n_{A}$ is the dimensionality of $\mathcal{H}_{A}$, the set $\left\{q_{i}\right\}$ is a probability distribution (i.e., $q_{i} \geq 0, \sum_{i} q_{i}=1$ ), and $\varrho_{B \mid i}$ are arbitrary states of subsystem $B$. The classical-quantum states (1) form a non convex set $\mathcal{C} \mathcal{Q}$, the convex hull of which is the set of all separable states. This means that there are separable states which are not classical-quantum. For pure states, however, classicality is equivalent to separability, since a pure state is classical-quantum if and only if it is a product state. Therefore quantum correlations must coincide with entanglement on pure states.

The evaluation of the entropic quantum discord is a highly nontrivial challenge, even when one restricts to the simplest case of two qubits (see e.g. Refs. [4 [6]). Geometric measures of quantum discord provide alternative ways to the entropic discord for quantifying quantum correlations in bipartite systems [7-17]. These measures offer the advantage of easier computability. Most of them have operational interpretations in terms of state distinguishability. On the other hand, they depend on a specific choice of some distance $d$ on the set of quantum states of the bipartite system $A B$. The most common choices are: 1) the trace distance $d_{\operatorname{Tr}}$ and Hilbert-Schmidt distance $d_{\mathrm{HS}}$, defined

\footnotetext{
* wojciech.roga@gmail.com

† Dominique.Spehner@ujf-grenoble.fr

† fabrizio.illuminati@gmail.com
} 
respectively as

$$
d_{\operatorname{Tr}}(\varrho, \sigma) \equiv\|\varrho-\sigma\|_{\operatorname{Tr}} \equiv \operatorname{Tr}|\varrho-\sigma| \quad, \quad d_{\mathrm{HS}}(\varrho, \sigma) \equiv\|\varrho-\sigma\|_{\mathrm{HS}} \equiv \sqrt{\operatorname{Tr}|\varrho-\sigma|^{2}}
$$

where $\varrho$ and $\sigma$ are two arbitrary states of $A B$ and $|X| \equiv \sqrt{X^{\dagger} X}$ is the modulus of the operator $X$; 2) the Bures distance [18, 19]

$$
d_{\mathrm{Bu}}(\varrho, \sigma) \equiv(2-2 \sqrt{F(\varrho, \sigma)})^{\frac{1}{2}} \quad, \quad F(\varrho, \sigma) \equiv\|\sqrt{\sigma} \sqrt{\varrho}\|_{\operatorname{Tr}}^{2}=\left(\operatorname{Tr}(\sqrt{\varrho} \sigma \sqrt{\varrho})^{\frac{1}{2}}\right)^{2},
$$

where $F(\varrho, \sigma)$ is the Uhlmann fidelity between $\varrho$ and $\sigma ; 3)$ the quantum Hellinger distance, called "Hellinger distance" for brevity in the sequel, which is defined as

$$
d_{\mathrm{He}}(\varrho, \sigma) \equiv\|\sqrt{\varrho}-\sqrt{\sigma}\|_{\mathrm{HS}}=(2-2 \operatorname{Tr} \sqrt{\varrho} \sqrt{\sigma})^{\frac{1}{2}} .
$$

For each of these distances, three major classes of geometric measures have been introduced in recent years:

I) The requirement that quantum correlations must vanish on the classical-quantum states has been exploited in Refs. [7, 9, 10, 14, 20 23] to define the geometric discord, equal to the square distance from a given state $\varrho$ of $A B$ to the set $\mathcal{C Q}$ of classical-quantum states:

$$
D_{A}^{G}(\varrho) \equiv \min _{\sigma_{\mathrm{cq}} \in \mathcal{C} \mathcal{Q}} d\left(\varrho, \sigma_{\mathrm{cq}}\right)^{2} .
$$

II) The measurement-induced geometric discord is defined by minimizing over all local projective measurements on $A$ the square distance between $\varrho$ and the corresponding post-measurement state in the absence of readout [20]:

$$
D_{A}^{M}(\varrho) \equiv \min _{\left\{\Pi_{i}^{A}\right\}} d\left(\varrho, \varrho_{\mathrm{p} . \mathrm{m} .}^{\left\{\Pi^{A}\right\}}\right)^{2} \quad, \quad \varrho_{\mathrm{p} . \mathrm{m} .}^{\left\{\Pi^{A}\right\}}=\sum_{i=1}^{n_{A}} \Pi_{i}^{A} \otimes \mathbb{1} \varrho \Pi_{i}^{A} \otimes \mathbb{1} .
$$

The minimum is taken over all families $\left\{\Pi_{i}^{A}\right\}$ of rank-one orthogonal projectors on $\mathcal{H}_{A}$. The quantity $D_{A}^{M}(\varrho)$ characterizes the distinguishability between $\varrho$ and the corresponding state after an arbitrary local von Neumann measurement on $A$. Since the output of such a measurement is always a classical-quantum state, one has $D_{A}^{G}(\varrho) \leq D_{A}^{M}(\varrho)$ for any $\varrho$. This inequality is an equality if $d$ is the Hilbert-Schmidt distance [20]. For the trace distance, the geometric discord and measurement-induced geometric discord coincide only if $A$ is a qubit (see Ref. [21], where explicit counter-examples for higher dimensional subsystems $A$ is also reported). For the Bures and Hellinger distances, $D_{A}^{G}$ and $D_{A}^{M}$ are in general different, irrespective of the space dimension $n_{A}$ (see Sec. $\mathrm{V}$ below).

III) Imposing the fundamental requirement that quantum correlations should be invariant under local changes of basis, one can introduce the discord of response, defined as [11, 13, 24.

$$
D_{A}^{R}(\varrho) \equiv \frac{1}{\mathcal{N}} \min _{U_{A} \in \mathcal{U}_{\Lambda}} d\left(\varrho, U_{A} \otimes \mathbb{1} \varrho U_{A}^{\dagger} \otimes \mathbb{1}\right)^{2},
$$

where the minimum is taken over all local unitary operators $U_{A}$ on $\mathcal{H}_{A}$ separated from the identity by the condition of having a fixed non-degenerated spectrum $\Lambda=\left\{e^{2 \mathrm{i} \pi j / n_{A}} ; j=1, \ldots, n_{A}\right\}$ given by the roots of unity (see Ref. [11] for a thorough discussion on the choice of the spectrum). Hereafter we denote by $\mathcal{U}_{\Lambda}$ the family of such unitaries with spectrum $\Lambda$. The normalization factor $\mathcal{N}$ in Eq. (7) is equal to $\mathcal{N}=4$ for the trace distance and $\mathcal{N}=2$ for the Bures, Hilbert-Schmidt, and Hellinger distances. As we shall see below, the normalization is such that $D_{A}^{R}(\varrho)$ has maximal value equal to unity. The discord of response characterizes how distinguishable is the locally unitarily perturbed state from the original one. Alternatively, it can be seen as a measure of the sensitivity of the state $\varrho$ to local unitary perturbations. For the Hilbert-Schmidt distance it holds $D_{A}^{R}(\varrho)=2 D_{A}^{G}(\varrho)$ when $A$ is a qubit $[11,24$.

Hereafter, we omit for simplicity the lower subscript $A$ on the discord $D$, as we will always take $A$ as the reference subsystem. The chosen distance is indicated explicitly. For instance, $D_{\mathrm{Tr}}^{G}, D_{\mathrm{HS}}^{G}, D_{\mathrm{Bu}}^{G}$, and $D_{\mathrm{He}}^{G}$ denote the geometric discords defined via the trace, Hilbert-Schmidt, Bures, and Hellinger distances, respectively. Let us note that another class of geometric measures, called the surprisal of measurement recoverability, has been studied recently in Ref. [25]. It is given in terms of the minimal distance of $\varrho$ to the subset formed by all transformations of $\varrho$ under entanglement breaking channels acting on $A$.

Besides the fact that the discords defined in Eqs. (5)-(7) are typically easier to compute than the entropic quantum discord of Refs. [1 [3], one of the main appealing features of the geometric approach is that it contains additional 
information. More specifically, let us consider a state $\varrho$ in the set $\mathcal{S}_{A B}$ of all quantum states of $A B$ and a distinguished subset of $\mathcal{S}_{A B}$, which might coincide with I) the subset $\mathcal{C} \mathcal{Q}$ of classical-quantum states, or II) the subset formed by all post-measurement states obtained from $\varrho$ through local von Neumann measurements on $A$, or III) the subset formed by all local unitary transformations of $\varrho$ with unitaries in $\mathcal{U}_{\Lambda}$. Then the state belonging to this subset that is closest to $\varrho$ for the distance $d$ provides some useful geometrical information about $\varrho$, which goes beyond the sole knowledge of the value of the distance between $\varrho$ and this closest state. For instance, it has been proposed in Ref. [12] to measure classical correlations in $\varrho$ by determining the minimal distance between a product state and a closest classical-quantum state to $\varrho$. The geometrical information can also be useful when considering dissipative dynamical evolutions. For instance, one can get some insight on the efficiency of the dynamical process in changing the amount of quantum correlations by comparing the physical trajectory $t \mapsto \varrho_{t}$ in $\mathcal{S}_{A B}$ with the geodesics connecting $\varrho_{t}$ to its closest classical-quantum state(s).

Another important feature of the geometric measures, which is related to the distinguishability of quantum states, concerns their operational interpretations. Indeed, various instances of these measures turn out to be valuable figures of merit in the context of quantum technology protocols [26], including quantum illumination 27, 28], quantum metrology and phase estimation [29], quantum refrigeration [30], and quantum local uncertainty [31]. In particular, the discord of response enjoys a beautiful operational interpretation in terms of the probability of error in protocols of quantum reading and quantum illumination [32].

The aim of this paper is to develop a systematic theory and exact mathematical characterization, quantification, and comparison between the geometric measures according to the different definitions in Eqs. (5)-(17) and to the different distances introduced above. We establish algebraic relations and inequalities holding between them. In particular, we provide some bounds on the geometric discord $D_{\mathrm{Bu}}^{G}$ for the Bures distance in terms of the corresponding discord for the Hellinger distance, and show that the latter is simply related to the Hilbert-Schmidt geometric discord for the square root of the state. Thanks to this relation, the Hellinger geometric discord $D_{\mathrm{He}}^{G}$ is fully computable; we illustrate this point by giving a closed expression for arbitrary qubit-qudit states. For a fixed distance, we also bound $D^{M}$ and $D^{R}$ in terms of $D^{G}$ and $D^{M}$ in terms of $D^{R}$. Bounds between $D_{\mathrm{Tr}}^{R}, D_{\mathrm{Bu}}^{R}$, and $D_{\mathrm{He}}^{R}$ are obtained as well. We show that some of these bounds are tight. In the particular case where the reference subsystem $A$ is a qubit (the other subsystem being arbitrary), we show that $D_{\operatorname{Tr}}^{G}=D_{\operatorname{Tr}}^{M}=D_{\operatorname{Tr}}^{R}$ and derive an exact algebraic relation between $D^{R}$ and $D^{G}$ holding for both the Bures and the Hellinger distances. Remarkably, this relation has the same form for the two metrics. We also describe the closest classical-quantum state and closest post-measurement state of a given bipartite state $\varrho$ and obtain the values taken by $D^{G}, D^{M}$, and $D^{R}$ on pure states for these two metrics.

Collecting the above results, we establish that the Hellinger geometric discord $D_{\mathrm{He}}^{G}$ and the Hellinger discord of response $D_{\mathrm{He}}^{R}$ provide the first two instances of measures of quantum correlations that are fully computable, reliable - since they satisfy all the basic Axioms that must be obeyed by a bona fide measure of quantum discord (which are detailed below) -, and operationally viable in terms of distinguishability of quantum states.

A further way to compare the measures defined in Eqs. (15)-(17) is to study the maximally quantum-correlated states at fixed global state purity for the different measures. We obtain analytical expressions for the maximal discord of response of two-qubit states as a function of their purity for the trace and Hellinger distances and compare our results with those found previously in the literature for the Hilbert-Schmidt and Bures metrics.

Finally, we discuss applications of the geometric measures to the problem of quantumness breaking channels. We determine a necessary condition for a local channel to destroy completely the quantum correlations of any bipartite state.

Before going into the detailed presentation and discussion of our results, it is worth recalling what we exactly mean by a bona fide measure of quantum correlations. Following previous works [5, 11, 14, 15, 29, 31], we stipulate that such a measure must be a non-negative function $D$ on the set of quantum states of the bipartite system $A B$ fulfilling the following four basic Axioms:

(i) $D$ vanishes on classical-quantum states and only on such states;

(ii) $D$ is invariant under local unitary transformations $\varrho \mapsto U_{A} \otimes U_{B} \varrho U_{A}^{\dagger} \otimes U_{B}^{\dagger}$ (here $U_{A}$ and $U_{B}$ are unitaries acting on subsystems $A$ and $B$, respectively);

(iii) $D$ is monotonically non increasing under local Completely Positive Trace Preserving (CPTP) maps acting on subsystem $B$;

(iv) $D$ reduces to an entanglement monotone on pure states.

Axioms (i-iv) are satisfied in particular by the entropic quantum discord. Here, we point out that proper measures of quantum correlations should also obey the following additional requirement (which is also fulfilled by the entropic discord) [15]:

(v) if the dimension $n_{A}$ of $\mathcal{H}_{A}$ is smaller or equal to the dimension $n_{B}$ of the space $\mathcal{H}_{B}$ of $B$, then $D(\varrho)$ is maximum if and only if $\varrho$ is maximally entangled, that is, $\varrho$ has maximal entanglement of formation $E_{\mathrm{EoF}}(\varrho)=\ln n_{A}$. 
Let us note that Axioms (iii) and (iv) imply that, when $n_{A} \leq n_{B}, D$ is maximum on maximally entangled pure states, i.e., if $\varrho$ is a maximally entangled pure state then $D(\varrho)=D_{\max }[16$. This follows from the facts that a function $D$ on $\mathcal{S}_{A B}$ satisfying (iii) is maximal on pure states if $n_{A} \leq n_{B}$ [33] and that any pure state can be obtained from a maximally entangled pure state via a LOCC [15]. Thus, if Axioms (i-iv) are satisfied, the additional requirement in Axiom (v) is essentially that $D(\varrho)=D_{\max }$ holds only for the maximally entangled states $\varrho$.

It has been shown in previous works [11, 15] that the geometric discord $D_{\mathrm{Bu}}^{G}$ and discord of response $D_{\mathrm{Bu}}^{R}$ satisfy Axioms (i)-(iv) for the Bures distance, and hence are bona fide measures of quantum correlations. In this paper, we will prove that this is also the case for the three measures $D_{\mathrm{He}}^{G}, D_{\mathrm{He}}^{M}$, and $D_{\mathrm{He}}^{R}$ based on the Hellinger distance, as well as for the Bures measurement-induced geometric discord $D_{\mathrm{Bu}}^{M}$ and trace discord of response $D_{\mathrm{Tr}}^{R}$. In contrast, it is known that $D_{\mathrm{HS}}^{G}=D_{\mathrm{HS}}^{M}$ and $D_{\mathrm{HS}}^{R}$ do not fulfill Axiom (iii) because of the lack of monotonicity of the Hilbert-Schmidt distance under CPTP maps (an explicit counter-example is given in Ref. 34] for $D_{\mathrm{HS}}^{G}$ and applies to $D_{\mathrm{HS}}^{R}$ as well). Therefore, the use of the Hilbert-Schmidt distance in the definitions of Eqs. (5)-(7) can and does lead to unphysical predictions. Considering the distances $d_{p}$ associated to the $p$-norms $\|X\|_{p} \equiv\left(\operatorname{Tr}|X|^{p}\right)^{1 / p}$, one has that for $p>1, d_{p}$ is not contractive under CPTP maps [35] (see also Ref. [36] for a counter-example for $p=2$, which also holds for any $p>1$ ). This is why the distances $d_{p}$ cannot be used to define measures of quantumness apart from the case $p=1$, corresponding to the contractive trace distance, while for $p=2$ the non-contractive Hilbert-Schmidt distance is well tractable and thus used to establish bounds on the bona fide geometric measures.

Regarding our last Axiom (v), the only result established so far in the literature concerns the Bures geometric discord [9, 15]. We will demonstrate below that all the other measures based on the trace, Bures, and Hellinger distances also satisfy this axiom. Our proofs are valid for arbitrary (finite) space dimensions $n_{A}$ and $n_{B}$ of subsystems $A$ and $B$, excepted for $D_{\mathrm{He}}^{G}$, for which they are restricted to the special cases $n_{A}=2,3$, and for $D_{\mathrm{He}}^{M}, D_{\mathrm{Tr}}^{G}$, and $D_{\mathrm{Tr}}^{M}$, for which they are restricted to $n_{A}=2$.

The paper is organized as follows. Given its length and the wealth of mathematical relations and bounds that we have determined, we begin by summarizing the main results in Section II We first give general expressions of the geometric measures for the Bures and Hellinger distances, which are convenient starting points to compare them (see Sec. IA . We then report in some synoptic Tables the various relations and bounds satisfied by $D^{G}$, $D^{M}$, and $D^{R}$ for the trace, Hilbert-Schmidt, Bures, and Hellinger distances (see Sec. IIB). Closed expressions for the Hellinger geometric discord and Hellinger discord of response for arbitrary qubit-qudit states are obtained in Sec. IIC thereby illustrating the computability of these two measures. A detailed comparison of all the geometric measures in the specific case of qubit-qudit systems (i.e., for $n_{A}=2$ and $n_{B} \geq 2$ ) is provided in Section IID where we derive from the synoptic Tables a large set of relations and bounds. We discuss there which bounds are tight. For the sake of completeness, we recall in Section III the definitions of the entropic quantum discord and local quantum uncertainty (see Sec. IIIA), some known bounds between the four aforementioned distances (see Sec. IIIB), and the main arguments and results from the literature enabling to show that $D^{G}, D^{M}$, and $D^{R}$ are bona fide measures of quantum correlations for the trace, Bures, and Hellinger distances (see Sec. IIC). We also recall in this section the link between the Bures geometric discord and a quantum state discrimination task 9] (see Sec. IIID). In Section IV we study the geometric discords, prove the identities and bounds reported in Table [] and present further results for the Hellinger geometric discord. Section $\nabla$ is devoted to the study of the measurement-induced geometric discord. The results summarized in Table $\amalg$ are proven in this section for the Bures and Hellinger distances. In Section VI we study the discord of response and prove the nontrivial relations and bounds reported in Table III In Section VII we study the maximal quantum correlations at fixed purity according to the different discords of response and discuss the different orderings that they induce on quantum states. The problem of quantumness breaking channels is addressed in Section VIII. Finally, we present a short discussion and our conclusions in Section IX. The four appendices report the technical proofs of some results stated in Sections VI, VII and VIII.

\section{SYNOPSIS: SUMMARY OF MAIN RESULTS}

\section{A. General expressions for the geometric measures: Hellinger and Bures distances}

Let us first restrict our attention to the Hellinger and Bures distances. We will show in the subsequent sections that the three geometric measures $D^{G}$ (geometric discord), $D^{M}$ (measurement-induced geometric discord), and $D^{R}$ (discord of response) are obtained by maximizing or minimizing a given trace over all orthonormal bases $\left\{\left|\alpha_{i}\right\rangle\right\}$ of the 
reference subsystem space $\mathcal{H}_{A}$. In the case of the Hellinger distance, we have

$$
\begin{aligned}
& D_{\mathrm{He}}^{G}(\varrho)=2-2 \max _{\left\{\left|\alpha_{i}\right\rangle\right\}}\left\{\sum_{i=1}^{n_{A}} \operatorname{Tr}_{B}\left\langle\alpha_{i}|\sqrt{\varrho}| \alpha_{i}\right\rangle^{2}\right\}^{\frac{1}{2}}, \\
& D_{\mathrm{He}}^{M}(\varrho)=2-2 \max _{\left\{\left|\alpha_{i}\right\rangle\right\}} \sum_{i=1}^{n_{A}} \operatorname{Tr}_{B}\left\langle\alpha_{i}|\sqrt{\varrho}| \alpha_{i}\right\rangle \sqrt{\left\langle\alpha_{i}|\varrho| \alpha_{i}\right\rangle}, \\
& D_{\mathrm{He}}^{R}(\varrho)=2 \min _{\left\{\left|\alpha_{i}\right\rangle\right\}} \sum_{i, j=1}^{n_{A}} \sin ^{2}\left(\frac{\pi(i-j)}{n_{A}}\right) \operatorname{Tr}_{B}\left|\left\langle\alpha_{i}|\sqrt{\varrho}| \alpha_{j}\right\rangle\right|^{2} .
\end{aligned}
$$

The derivation of Eq. (8) is the content of Theorem 11. proved in Section [V] below. Equation (9) is a rather direct consequence of the definitions and Eq. (10) is derived in Appendix A. Before providing the corresponding expressions for the Bures distance, let us introduce the probabilities $\eta_{i}$ and states $\varrho_{i}$ depending on the orthonormal basis $\left\{\left|\alpha_{i}\right\rangle\right\}$ defined by

$$
\eta_{i}=\left\langle\alpha_{i}\left|\varrho_{A}\right| \alpha_{i}\right\rangle \quad, \quad \varrho_{i}=\eta_{i}^{-1} \sqrt{\varrho}\left|\alpha_{i}\right\rangle\left\langle\alpha_{i}\right| \otimes \mathbb{1} \sqrt{\varrho} \quad, \quad i=1, \ldots, n_{A},
$$

where $\varrho_{A}=\operatorname{Tr}_{B}(\varrho)$ is the reduced state of $A$. It has been shown in Refs. 9, 15] that the Bures geometric discord is obtained by maximizing over all $\left\{\left|\alpha_{i}\right\rangle\right\}$ 's the maximal success probability $P_{\mathrm{S}}^{\text {opt v.N. }}\left(\left\{\varrho_{i}, \eta_{i}\right\}\right)$ to discriminate the states $\varrho_{i}$ with prior probabilities $\eta_{i}$ by means of von Neumann measurements with projectors of rank $n_{B}$. The reader unfamiliar with quantum state discrimination theory can find the definition of this success probability in Section IIID below. It turns out that $D_{\mathrm{Bu}}^{M}$ and $D_{\mathrm{Bu}}^{R}$ can also be expressed in terms of $\varrho_{i}$ and $\eta_{i}$. More precisely, one has (see Sections IID $\mathrm{VB}$ and $\mathrm{VIB}$ :

$$
\begin{aligned}
& D_{\mathrm{Bu}}^{G}(\varrho)=2-2 \max _{\left\{\left|\alpha_{i}\right\rangle\right\}} \sqrt{P_{\mathrm{S}}^{\text {opt v.N. }}\left(\left\{\varrho_{i}, \eta_{i}\right\}\right)}, \\
& D_{\mathrm{Bu}}^{M}(\varrho)=2-2 \max _{\left\{\left|\alpha_{i}\right\rangle\right\}} \operatorname{Tr} \sqrt{\sum_{j=1}^{n_{A}} \eta_{j}^{2} \varrho_{j}^{2}}, \\
& D_{\mathrm{Bu}}^{R}(\varrho)=1-\max _{\left\{\left|\alpha_{i}\right\rangle\right\}} \operatorname{Tr}\left|\sum_{j=1}^{n_{A}} \eta_{j} e^{-\mathrm{i} \frac{2 \pi j}{n_{A}}} \varrho_{j}\right| .
\end{aligned}
$$

By using these expressions, the values of $D^{G}, D^{M}$, and $D^{R}$ for a bipartite pure state can be determined explicitly in terms of the Schmidt coefficients of this state. These values are given in Tables IIIII and are all entanglement monotones. This enable us to show that for the Bures and Hellinger distances, $D^{G}$, $D^{M}$, and $D^{R}$ are bona fide measures of quantum correlations satisfying the Axioms (i-iv) above, as detailed in Section IIIC. In Appendix B we show that these measures obey Axiom (v) as well, although a proof for arbitrary space dimensions $n_{A}$ is still lacking in a few cases (see Tables IIII for more detail). In contrast, for the Hilbert-Schmidt distance, $D^{G}$, $D^{M}$, and $D^{R}$ do not fulfill Axiom (iii) and hence are not bona fide measures of quantum correlations.

\section{B. Exact relations and bounds between the geometric measures: arbitrary bipartite systems}

The Tables IIII summarize most of our results on the properties of the geometric measures of quantum correlations, most notably the relations and bounds between them which are derived in Theorems 2, 3, 5, and 7, 12, below. When not stated otherwise, all identities and bounds hold for arbitrary finite dimensions $n_{A}$ and $n_{B}$ of the Hilbert spaces $\mathcal{H}_{A}$ and $\mathcal{H}_{B}$. Many bounds are non trivial and are established by using the general expressions given in Eqs. (8)-(14). In addition, we also report in Tables [III some straightforward but important consequences of general inequalities between the trace, Hilbert-Schmidt, Bures, and Hellinger distances, which are recalled in Section IIBbelow. Tables III do not contain all such trivial bounds, so we write them explicitly here for the geometric discord:

$$
\begin{aligned}
& \frac{1}{n_{A} n_{B}} D_{\operatorname{Tr}}^{G}(\varrho) \leq D_{\mathrm{HS}}^{G}(\varrho) \leq D_{\operatorname{Tr}}^{G}(\varrho) \\
& D_{\mathrm{Bu}}^{G}(\varrho)^{2} \leq D_{\mathrm{He}}^{G}(\varrho)^{2} \leq D_{\operatorname{Tr}}^{G}(\varrho) \leq 2 g\left(D_{\mathrm{Bu}}^{G}(\varrho)\right),
\end{aligned}
$$

where we have introduced the function $g(d) \equiv 2 d-d^{2} / 2$. The same inequalities hold for the measurement-induced geometric discord and the discord of response, except that the latter appears multiplied by an extra normalization factor $\mathcal{N}=4$ for the trace distance and $\mathcal{N}=2$ for the Hilbert-Schmidt, Bures, and Hellinger distances. This is a trivial consequence of the normalization introduced in the definition of $D^{R}$, see Eq. (7). 


\begin{tabular}{|c|c|c|c|c|}
\hline & \multicolumn{4}{|c|}{ Geometric discord $D^{G}$} \\
\hline Distance & Bures & Hellinger & Trace & Hilbert Schmidt \\
\hline $\begin{array}{l}\text { Proper measure of } \\
\text { quantum correlations }\end{array}$ & $\checkmark$ & $\checkmark$ & proved for $n_{A}=2$ & no \\
\hline Satisfies Axiom (v) & $\checkmark$ & proved for $n_{A}=2,3$ & proved for $n_{A}=2$ & \\
\hline $\begin{array}{l}\text { Maximal value } \\
\text { if } n_{A} \leq n_{B}\end{array}$ & $2-2 / \sqrt{n_{A}}$ & $2-2 / \sqrt{n_{A}}$ & 1 for $n_{A}=2$ & \\
\hline Value for pure states & $2-2 \sqrt{\mu_{\max }}$ & $2-2 K^{-\frac{1}{2}}$ & $?$ & $1-K^{-1}$ \\
\hline $\begin{array}{c}\text { Relations and } \\
\text { cross inequalities }\end{array}$ & \multicolumn{4}{|c|}{$2-2 \sqrt{1-D_{\mathrm{He}}^{G}(\varrho) / 2} \leq D_{\mathrm{Bu}}^{G}(\varrho) \leq D_{\mathrm{He}}^{G}(\varrho)=2-2 \sqrt{1-D_{\mathrm{HS}}^{G}(\sqrt{\varrho})}$} \\
\hline $\begin{array}{l}\text { Computability } \\
\text { for two qubits }\end{array}$ & Bell-diagonal states & all states & $\begin{array}{l}\text { X-states } \\
\text { quantum-classical states }\end{array}$ & all states \\
\hline $\begin{array}{l}\text { Closest classical- } \\
\text { quantum state }\end{array}$ & $\begin{array}{l}\text { given by Eq. } 51 \text { ) } \\
\text { (Eq. } 59 \text { for pure states) }\end{array}$ & $\begin{array}{l}\text { given by Eq. } 57 \\
\text { (Eq. } \sqrt{55} \text { for pure states) }\end{array}$ & $?$ & $\begin{array}{l}\text { given by Eq. } 72 \text {. } \\
\text { (Eq. } 81 \text { for pure states) }\end{array}$ \\
\hline
\end{tabular}

TABLE I: Summary of the original results from Section [V] as well as of previous results obtained in Refs. [7, 9, 14], for the geometric discord with the Bures, Hellinger, trace, and Hilbert-Schmidt distances. Here $n_{A}$ denotes the Hilbert space dimension of the reference subsystem $A$. The quantities $\mu_{\max }=\max \left\{\mu_{i}\right\}$ and $K=\left(\sum_{i} \mu_{i}^{2}\right)^{-1}$ are, respectively, the maximal Schmidt coefficient and the Schmidt number of a pure state. The question marks indicate unsolved problems.

\begin{tabular}{|c|c|c|c|c|}
\hline & \multicolumn{4}{|c|}{ Measurement-induced geometric discord $D^{M}$} \\
\hline Distance & Bures & Hellinger & Trace & Hilbert Schmidt \\
\hline $\begin{array}{l}\text { Proper measure of } \\
\text { quantum correlations }\end{array}$ & $\checkmark$ & $\checkmark$ & $\checkmark$ & no \\
\hline Satisfies Axiom (v) & $\checkmark$ & for $n_{A}=2$ (conjecture) & proved for $n_{A}=2$ & \\
\hline $\begin{array}{l}\text { Maximal value } \\
\text { if } n_{A} \leq n_{B}\end{array}$ & $2-2 / \sqrt{n_{A}}$ & $2-2 / \sqrt{n_{A}}$ & $\left(2-2 / n_{A}\right)^{2}$ & \\
\hline Value for pure states & $2-2 K^{-\frac{1}{2}}$ & $2-2 \sum_{i} \mu_{i}^{\frac{3}{2}}$ & see Theorem 3.3 in [16] & $1-K^{-1}$ \\
\hline $\begin{array}{l}\text { Comparison with the } \\
\text { geometric discord }\end{array}$ & $D_{\mathrm{Bu}}^{G} \leq D_{\mathrm{Bu}}^{M} \leq 2 D_{\mathrm{Bu}}^{G}-\frac{1}{2}\left(D_{\mathrm{Bu}}^{G}\right)^{2}$ & $D_{\mathrm{He}}^{G} \leq D_{\mathrm{He}}^{M} \leq 2 D_{\mathrm{He}}^{G}-\frac{1}{2}\left(D_{\mathrm{He}}^{G}\right)^{2}$ & $\begin{cases}D_{\mathrm{Tr}}^{M}=D_{\mathrm{Tr}}^{G} & \text { for } n_{A}=2 \\
D_{\mathrm{Tr}}^{M} \geq D_{\mathrm{Tr}}^{G} & \text { for } n_{A}>2\end{cases}$ & $D_{\mathrm{HS}}^{M}=D_{\mathrm{HS}}^{G}$ \\
\hline $\begin{array}{l}\text { Computability } \\
\text { for two qubits }\end{array}$ & ? & $?$ & $\begin{array}{l}\text { X-states } \\
\text { quantum-classical states }\end{array}$ & all states \\
\hline $\begin{array}{l}\text { Closest post-measu- } \\
\text { rement state }\end{array}$ & $\begin{array}{c}\text { for pure states, given } \\
\text { by Eq. } 81\end{array}$ & $\begin{array}{c}\text { for pure states, given } \\
\text { by Eq. } 81\end{array}$ & $\begin{array}{c}\text { for pure states, given } \\
\text { by Eq. 81] }\end{array}$ & $\begin{array}{l}\text { given by Eq. 72 (Eq. } \\
\text { 81) for pure states) }\end{array}$ \\
\hline
\end{tabular}

TABLE II: Summary of the original results from Section $\nabla$ as well as of previous results obtained in Refs. 7, 14, 16, 20, 21], for the measurement-induced geometric discord with the Bures, Hellinger, trace, and Hilbert-Schmidt distances. The notations are the same as the ones introduced and explained in the caption of Table I

\section{Computability of the Hellinger geometric discord and Hellinger discord of response}

Let us point out the simple expressions found in Tables \and $\llbracket$ for the Hellinger geometric discord and discord of response in terms of the corresponding measures for the Hilbert-Schmidt distance of the square root of $\varrho$,

$$
D_{\mathrm{He}}^{G}(\varrho)=2-2 \sqrt{1-D_{\mathrm{HS}}^{G}(\sqrt{\varrho})} \quad, \quad D_{\mathrm{He}}^{R}(\varrho)=D_{\mathrm{HS}}^{R}(\sqrt{\varrho}) .
$$

The first identity is the content of Theorem 2 below and the second one is a trivial consequence of the definitions, see Eqs. (41) and (7). As a result, since the geometric measures with Hilbert-Schmidt distance are known to be easy to compute [5, 7, 20], so are the Hellinger geometric discord and discord of response. We emphasize that $D_{\mathrm{He}}^{G}$ and $D_{\mathrm{He}}^{R}$ are bona fide measures of quantum correlations satisfying the basic Axioms (i-iv) of Sec. I] as opposed to the Hilbert-Schmidt measures which do not obey the monotonicity Axiom (iii). Hence $D_{\mathrm{He}}^{G}$ and $D_{\mathrm{He}}^{R}$ have the appealing feature of being at the same time physically reliable and easy to compute.

In fact, we can do better and determine directly with the help of Eq. (8) an explicit expression for the Hellinger geometric discord whenever $A$ is a qubit and $B$ is an arbitrary system with a $n_{B}$-dimensional Hilbert space (qudit). Note that in this case $D_{\mathrm{He}}^{G}$ and $D_{\mathrm{He}}^{R}$ are simply related to each other, as well as when $A$ is a qutrit (see Table III). Hence, if one is able to compute $D_{\mathrm{He}}^{G}$ then the computability of $D_{\mathrm{He}}^{R}$ for $n_{A}=2,3$ immediately follows. In the case $n_{A}=2$, let us introduce the vector $\vec{\sigma}$ formed by the three Pauli matrices acting on $A$. Similarly, let the vector $\vec{\gamma}$ be formed by the $\left(n_{B}^{2}-1\right)$ self-adjoint operators $\gamma_{p}$ acting on $B$ such that $\left\{\mathbb{1} / \sqrt{n_{B}}, \gamma_{p} / \sqrt{n_{B}}\right\}_{p=1}^{n_{B}^{2}-1}$ is an orthonormal basis of the Hilbert space of all $n_{B} \times n_{B}$ matrices. This means that $\operatorname{Tr} \gamma_{p}=0$ and $\operatorname{Tr} \gamma_{p} \gamma_{q}=n_{B} \delta_{p q}$ for any $p, q=1, \ldots, n_{B}^{2}-1$. 


\begin{tabular}{|c|c|c|c|c|c|}
\hline & & \multicolumn{4}{|c|}{ Discord of response $D^{R}$} \\
\hline \multicolumn{2}{|c|}{ Distance } & Bures & Hellinger & Trace & Hilbert Schmidt \\
\hline \multicolumn{2}{|c|}{$\begin{array}{l}\text { Proper measure of } \\
\text { quantum correlations }\end{array}$} & $\checkmark$ & $\checkmark$ & $\checkmark$ & no \\
\hline \multicolumn{2}{|c|}{ Satisfies Axiom (v) } & $\checkmark$ & $\checkmark$ & $\checkmark$ & no if $n_{B} \geq 2 n_{A}$ \\
\hline \multicolumn{2}{|c|}{$\begin{array}{l}\text { Maximal value } \\
\text { if } n_{A} \leq n_{B}\end{array}$} & 1 & 1 & 1 & 1 \\
\hline \multicolumn{2}{|c|}{ Value for pure states } & $1-\sqrt{1-E^{R}}$ & $E^{R}$ & $E^{R}$ & $E^{R}$ \\
\hline \multirow{3}{*}{$\begin{array}{l}\text { Comparison } \\
\text { with } D^{G}\end{array}$} & $n_{A}=2$ & $D_{\mathrm{Bu}}^{R}=2 D_{\mathrm{Bu}}^{G}-\frac{1}{2}\left(D_{\mathrm{Bu}}^{G}\right)^{2}$ & $D_{\mathrm{He}}^{R}=2 D_{\mathrm{He}}^{G}-\frac{1}{2}\left(D_{\mathrm{He}}^{G}\right)^{2}$ & $D_{\mathrm{Tr}}^{R}=D_{\mathrm{Tr}}^{G}$ & $D_{\mathrm{HS}}^{R}=2 D_{\mathrm{HS}}^{G}$ \\
\hline & $n_{A}=3$ & $1-\sqrt{1-\frac{1}{n_{A} n_{B}} \sin ^{2}\left(\frac{\pi}{n_{A}}\right)\left(D_{\mathrm{Bu}}^{G}\right)^{2}}$ & $D_{\mathrm{He}}^{R}=\frac{3}{2} D_{\mathrm{He}}^{G}-\frac{3}{8}\left(D_{\mathrm{He}}^{G}\right)^{2}$ & \multirow{2}{*}{$\begin{array}{c}\frac{1}{n_{A} n_{B}} \sin ^{2}\left(\frac{\pi}{n_{A}}\right) D_{\mathrm{Tr}}^{G} \\
\leq D_{\mathrm{Tr}}^{R} \leq \\
n_{A} n_{B} D_{\mathrm{Tr}}^{G}\end{array}$} & $D_{\mathrm{HS}}^{R}=\frac{3}{2} D_{\mathrm{HS}}^{G}$ \\
\hline & $n_{A}>3$ & $\frac{\leq D_{\mathrm{Bu}}^{K} \leq}{\sqrt{2 n_{A} n_{B}\left(2 D_{\mathrm{Bu}}^{G}-\frac{1}{2}\left(D_{\mathrm{Bu}}^{G}\right)^{2}\right)}}$ & $\begin{array}{c}\sin ^{2}\left(\frac{\pi}{n_{A}}\right)\left(2 D_{\mathrm{He}}^{G}-\frac{1}{2}\left(D_{\mathrm{He}}^{G}\right)^{2}\right) \\
\leq D_{\mathrm{He}}^{R} \leq 2 D_{\mathrm{He}}^{G}-\frac{1}{2}\left(D_{\mathrm{He}}^{G}\right)^{2}\end{array}$ & & $\begin{array}{l}2 \sin ^{2}\left(\frac{\pi}{n_{A}}\right) D_{\mathrm{HS}}^{G} \\
\leq D_{\mathrm{HS}}^{R} \leq 2 D_{\mathrm{HS}}^{G}\end{array}$ \\
\hline \multirow{3}{*}{$\begin{array}{l}\text { Comparison } \\
\text { with } D^{M}\end{array}$} & $n_{A}=2$ & $D_{\mathrm{Bu}}^{M} \leq 2-\sqrt{2} \sqrt{1+\left(1-D_{\mathrm{Bu}}^{R}\right)^{2}}$ & \multirow{3}{*}{$\sin ^{2}\left(\frac{\pi}{n_{A}}\right) D_{\mathrm{He}}^{M} \leq D_{\mathrm{He}}^{R}$} & $D_{\mathrm{Tr}}^{R}=D_{\mathrm{Tr}}^{M}$ & $D_{\mathrm{HS}}^{R}=2 D_{\mathrm{HS}}^{M}$ \\
\hline & $n_{A}=3$ & $D_{\mathrm{Bu}}^{M} \leq 2-\frac{2}{\sqrt{3}} \sqrt{1+2\left(1-D_{\mathrm{Bu}}^{R}\right)^{2}}$ & & $?$ & $D_{\mathrm{HS}}^{R}=\frac{3}{2} D_{\mathrm{HS}}^{M}$ \\
\hline & $n_{A}>3$ & $D_{\mathrm{Bu}}^{M} \leq 2-\frac{2}{\sqrt{n_{A}}}\left(1-D_{\mathrm{Bu}}^{R}\right)$ & & & $\begin{array}{l}2 \sin ^{2}\left(\frac{\pi}{n_{A}}\right) D_{\mathrm{HS}}^{M} \\
\leq D_{\mathrm{HS}}^{R} \leq 2 D_{\mathrm{HS}}^{M}\end{array}$ \\
\hline \multicolumn{2}{|c|}{$\begin{array}{c}\text { Cross inequalities } \\
\text { and relations } \\
\end{array}$} & \multicolumn{4}{|c|}{$D_{\mathrm{Bu}}^{R} \leq D_{\mathrm{He}}^{R} \leq 1-\left(1-D_{\mathrm{Bu}}^{R}\right)^{2} \quad, \quad\left(D_{\mathrm{He}}^{R}\right)^{2} \leq D_{\mathrm{Tr}}^{R} \leq 1-\left(1-D_{\mathrm{Bu}}^{R}\right)^{2} \quad, \quad D_{\mathrm{He}}^{R}(\varrho)=D_{\mathrm{HS}}^{R}(\sqrt{\varrho})$} \\
\hline \multicolumn{2}{|c|}{$\begin{array}{l}\text { Computability } \\
\text { for two qubits }\end{array}$} & Bell-diagonal states & all states & $\begin{array}{l}\text { X-states } \\
\text { quantum-classical states }\end{array}$ & all states \\
\hline
\end{tabular}

TABLE III: Summary of the original results from Section VI and Appendix $\mathrm{A}$ as well as of previous results obtained in Ref. 11], for the discord of response with the Bures, Hellinger, trace, and Hilbert-Schmidt distances. Here $E^{R}$ is the entanglement of response, see Eq. (43). The remaining notations are the same as the ones introduced and explained in the caption of Table I]

The square root of $\varrho$ can be decomposed as

$$
\sqrt{\varrho}=\frac{1}{\sqrt{2 n_{B}}}\left(t_{0} \mathbb{1} \otimes \mathbb{1}+\vec{x} \cdot \vec{\sigma} \otimes \mathbb{1}+\mathbb{1} \otimes \vec{y} \cdot \vec{\gamma}+\sum_{m=1}^{3} \sum_{p=1}^{n_{B}^{2}-1} t_{m p} \sigma_{m} \otimes \gamma_{p}\right)
$$

with $t_{0} \in[-1,1], \vec{x} \in \mathbb{R}^{3}$, and $\vec{y} \in \mathbb{R}^{n_{B}^{2}-1}$. We denote by $T$ the $3 \times\left(n_{B}^{2}-1\right)$ complex matrix with coefficients $t_{m p}$. The condition $\operatorname{Tr}(\sqrt{\varrho})^{2}=1$ entails $t_{0}^{2}+\|\vec{x}\|^{2}+\|\vec{y}\|^{2}+\operatorname{Tr}\left(T T^{T}\right)=1$ (here $T^{T}$ stands for the transpose of $T$ ). For any orthonormal basis $\left\{\left|\alpha_{i}\right\rangle\right\}_{i=0,1}$ for qubit $A$, one finds

$$
\sum_{i=0,1} \operatorname{Tr}\left[\left\langle\alpha_{i}|\sqrt{\varrho}| \alpha_{i}\right\rangle^{2}\right]=t_{0}^{2}+\|\vec{y}\|^{2}+\vec{u}^{T}\left(\vec{x} \vec{x}^{T}+T T^{T}\right) \vec{u}
$$

where we have introduced the unit vector $\vec{u}=\left\langle\alpha_{0}|\vec{\sigma}| \alpha_{0}\right\rangle=-\left\langle\alpha_{1}|\vec{\sigma}| \alpha_{1}\right\rangle$. Maximizing over all such vectors and using Eq. (8), we have

$$
D_{\mathrm{He}}^{G}(\varrho)=2-2 \sqrt{t_{0}^{2}+\|\vec{y}\|^{2}+k_{\max }},
$$

where $k_{\max }$ is the largest eigenvalue of the $3 \times 3$ matrix $K=\vec{x} \vec{x}^{T}+T T^{T}$. Therefore, the calculation of $D_{\mathrm{He}}^{G}(\varrho)$ is straightforward once one has determined the decomposition Eq. (18) of the square root of $\varrho$. The Hellinger geometric discord is thus easily computable on all qubit-qudit states.

The computability for qubit-qudit states was also noticed in Ref. [17] for a modified version of the Hellinger measurement-induced geometric discord, defined as

$$
\Delta_{\mathrm{He}}^{M}(\varrho)=\min _{\left\{\Pi_{i}^{A}\right\}}\left\|\sqrt{\varrho}-\sum_{i=1}^{n_{A}} \Pi_{i}^{A} \otimes \mathbb{1} \sqrt{\varrho} \Pi_{i}^{A} \otimes \mathbb{1}\right\|_{\mathrm{HS}}^{2},
$$

where the minimum is taken over all families $\left\{\Pi_{i}^{A}\right\}$ of rank-one orthogonal projectors on $\mathcal{H}_{A}$. When $A$ is a qubit, $\Delta_{\mathrm{He}}^{M}(\varrho)=D_{\mathrm{He}}^{R}(\varrho) / 2$ coincides with the Hellinger discord of response up to a factor of one half. In this case, $D_{\mathrm{He}}^{R}(\varrho)$ is also equal to the local quantum uncertainty (LQU) measure $\mathcal{U}_{A}^{\Lambda}(\varrho)$ for bipartite systems introduced in Ref. [31] (we refer the reader to Section $\amalg$ II for the definition of $\mathcal{U}_{A}^{\Lambda}(\varrho)$ ). This measure was evaluated explicitly for qubit-qudit states in Ref. [31]. From this result, one finds (see Sec. III A)

$$
D_{\mathrm{He}}^{R}(\varrho)=\mathcal{U}_{A}^{\Lambda}(\varrho)=2-2\left(t_{0}^{2}+\|\vec{y}\|^{2}+k_{\max }\right) .
$$

This expression is consistent with Eqs. (20) and the relation $D_{\mathrm{He}}^{R}(\varrho)=2 D_{\mathrm{He}}^{G}(\varrho)-D_{\mathrm{He}}^{G}(\varrho)^{2} / 2$ from Table III 

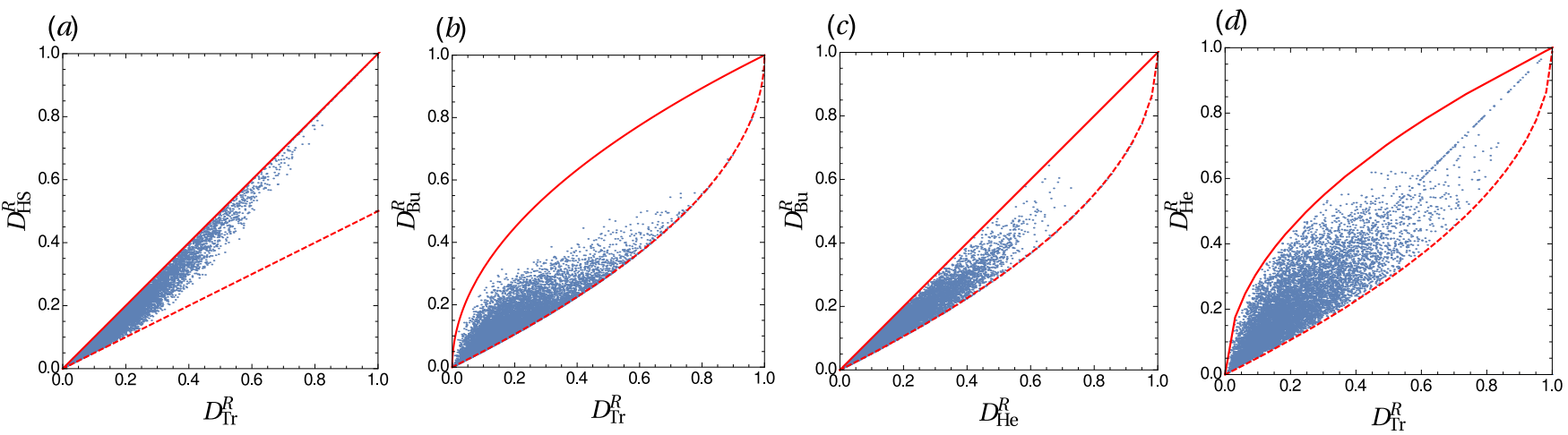

FIG. 1: Comparison of the discords of response based on the four distances introduced in Sect. [. The points represent 104 randomly generated two-qubit states (see main text for details). The lines correspond to equalities in the inequalities of Eqs. (24) and (27). (a) Hilbert-Schmidt and trace discords of response $D_{\mathrm{HS}}^{R}$ and $D_{\mathrm{Tr}}^{R}$. Red solid line $D_{\mathrm{HS}}^{R}=D_{\mathrm{Tr}}^{R}$ achieved for pure states, see Eq. (24); red dashed line: $D_{\mathrm{HS}}^{R}=D_{\mathrm{Tr}}^{R} / 2$, see Eq. (24). (b) Bures and trace discords of response $D_{\mathrm{Bu}}^{R}$ and $D_{\mathrm{Tr}}^{R}$. Red solid line: $D_{\mathrm{Bu}}^{R}=\sqrt{D_{\operatorname{Tr}}^{R}}$, see Eq. (27); red dashed line: $D_{\mathrm{Bu}}^{R}=1-\sqrt{1-D_{\mathrm{Tr}}^{R}} \Leftrightarrow D_{\mathrm{Tr}}^{R}=2 D_{\mathrm{Bu}}^{R}-\left(D_{\mathrm{Bu}}^{R}\right)^{2}$ achieved for pure states, see Eq. (27). (c) Bures and Hellinger discords of response $D_{\mathrm{Bu}}^{R}$ and $D_{\mathrm{He}}^{R}$. Red solid line: $D_{\mathrm{Bu}}^{R}=D_{\mathrm{He}}^{R}$, see Eq. (27); dotted line: $D_{\mathrm{Bu}}^{R}=1-\sqrt{1-D_{\mathrm{He}}^{R}}$, achieved for pure states, see Eq. (27). (d) Hellinger and trace discords of response $D_{\mathrm{He}}^{R}$ and $D_{\mathrm{Tr}}^{R}$. Red solid line: $D_{\mathrm{He}}^{R}=\sqrt{D_{\mathrm{Tr}}^{R}}$, see Eq. (27); red dashed line: $D_{\mathrm{He}}^{R}=1-\sqrt{1-D_{\mathrm{Tr}}^{R}} \Leftrightarrow D_{\mathrm{Tr}}^{R}=2 D_{\mathrm{He}}^{R}-\left(D_{\mathrm{He}}^{R}\right)^{2}$, see Eq. (27).

\section{Inequalities between the geometric measures: qubit-qudit systems}

In this subsection we consider the specific case of a reference subsystem $A$ being a qubit $\left(n_{A}=2\right)$, while subsystem $B$ is of arbitrary space dimension. We summarized in Tables IIIII an ample set of bounds holding between the geometric measures. It is important to establish whether these bounds are tight or not. A lower or upper bound on a measure $D$ varying in the interval $\left[0, D_{\max }\right]$ is said to be tight if for every value $d \in\left[0, D_{\max }\right]$, there exists a bipartite state $\varrho$ such that $D(\varrho)=d$ and $\varrho$ saturates the bound.

In general, proving that a bound is tight can be challenging. To get some insight into this problem, we have generated numerically random two-qubit states, computed $D^{M}$ and $D^{R}$ for the four distances considered in this paper, and drawn in Figs. 1,3 the corresponding distributions in the planes formed by the pairs of measures we wish to compare. The random two-qubit states of rank $k$ are obtained by taking the partial trace over a $k$-dimensional ancillary system of randomly generated pure states of the composed (two qubits + ancilla) system. The ensemble of pure states is distributed according to the unitarily invariant Fubini-Study measure on the projective space of the composed system [37]. The rank $k$ is chosen randomly. Consider the set of points formed by the values of a given pair of measures we wish to compare for all randomly generated states. When the line defined by the equality in a bound between these two measures is very close to the border of this set, we say that the bound is almost tight. Note that, although this gives an indication that the bound could be tight, the tightness property can only be established by finding analytically a family of states with arbitrary discord fulfilling the equality.

Our analytical bounds involve the following real increasing functions from $[0,2-\sqrt{2}]$ onto $[0,1]$ :

$$
g(d) \equiv 2 d-\frac{1}{2} d^{2} \quad, \quad h(d) \equiv 2 g(d)-(g(d))^{2} .
$$

The inverse of $g$ is $g^{-1}(d)=2-2 \sqrt{1-d / 2}$.

Comparison of the geometric measures for the trace and Hilbert Schmidt distances. From Tables [III we get

$$
D_{\mathrm{Tr}}^{G}=D_{\mathrm{Tr}}^{M}=D_{\mathrm{Tr}}^{R} \quad, \quad D_{\mathrm{HS}}^{G}=D_{\mathrm{HS}}^{M}=\frac{1}{2} D_{\mathrm{HS}}^{R} \quad, \quad \frac{1}{n_{B}} D_{\mathrm{Tr}}^{R} \leq D_{\mathrm{HS}}^{R} \leq D_{\mathrm{Tr}}^{R} .
$$

The equality between $D^{G}$ and $D^{M}$ for the trace and Hilbert-Schmidt distances is already known, see Refs. 21] and 20]. The relations $D_{\mathrm{Tr}}^{G}=D_{\operatorname{Tr}}^{R}$ and $D_{\mathrm{HS}}^{G}=D_{\mathrm{HS}}^{R} / 2$ are proven in Appendix $\mathrm{A}$. The first inequality in Eq. (24) is a trivial bound analog to Eq. (15). The second one is the content of Theorem 12 proven in Appendix $\mathrm{A}$,

The numerical results displayed in Fig. 1(a) indicate that the first inequality is unlikely to be tight in the whole range $[0,1]$, although it is almost optimal for weakly discordant states. The second inequality is saturated for pure states, as can be checked by using the values reported in Table III for such states. Hence this inequality is tight.

Comparison of the three geometric measures based on the Bures distance. The discords $D_{\mathrm{Bu}}^{G}, D_{\mathrm{Bu}}^{M}$, and $D_{\mathrm{Bu}}^{R}$ are ordered as follows:

$$
D_{\mathrm{Bu}}^{G} \leq D_{\mathrm{Bu}}^{M} \leq g^{-1} \circ h\left(D_{\mathrm{Bu}}^{G}\right) \leq D_{\mathrm{Bu}}^{R}=g\left(D_{\mathrm{Bu}}^{G}\right) \leq g\left(D_{\mathrm{Bu}}^{M}\right) \quad, \quad D_{\mathrm{Bu}}^{G} \leq(2-\sqrt{2}) D_{\mathrm{Bu}}^{R} .
$$


The first inequality is a straightforward consequence of the definitions of $D^{G}$ and $D^{M}$ and is almost tight for two qubits (see below). The second one is established by using $D_{\mathrm{Bu}}^{R}=g\left(D_{\mathrm{Bu}}^{G}\right)$ and $D_{\mathrm{Bu}}^{M} \leq 2-\left(2+2\left(1-D_{\mathrm{Bu}}^{R}\right)^{2}\right)^{1 / 2}$ from Table III It is saturated for pure states (see Theorem 11 below). The third inequality comes from $g^{-1} \circ h(d) \leq g(d)$ for any $d \in[0,2-\sqrt{2}]$. It is not tight (it is an equality for classical-quantum states only). The fourth inequality follows from the first one and the monotonicity of the function $g$. We find numerically that this inequality is almost tight for two-qubit states, see Fig. 3(a). This indicates that the same is true for the first inequality.

The last inequality in Eq. (25) can be proven by exploiting $D_{\mathrm{Bu}}^{R}=g\left(D_{\mathrm{Bu}}^{G}\right)$, the bound $d \leq(2-\sqrt{2}) g(d)$ for any $d \in[0,2-\sqrt{2}]$, and the fact that the highest value of $D_{\mathrm{Bu}}^{G}$ is $2-\sqrt{2}$ when $A$ is a qubit [9]. This inequality is not tight (it is saturated for classical-quantum and maximally entangled states only). It has been conjectured in Ref. [11] to hold for Bell-diagonal two-qubit states by relying on numerical investigations with randomly generated states. The above argument provide an analytical proof valid for arbitrary states of a bipartite system with $n_{A}=2$ and $n_{B} \geq 2$.

Let us also point out that closed expressions for $D_{\mathrm{Bu}}^{G}(\varrho)$ and $D_{\mathrm{Bu}}^{R}(\varrho)$ have been determined for Bell-diagonal states $\varrho$ in Refs. [8, 10] and Ref. [11], respectively. It is straightforward to verify that these results are related to each other by specializing $D_{\mathrm{Bu}}^{R}=g\left(D_{\mathrm{Bu}}^{G}\right)$ to the case of Bell-diagonal states.

Comparison of the geometric measures based on the Hellinger distance. Similarly, one has

$$
D_{\mathrm{He}}^{G} \leq D_{\mathrm{He}}^{M} \leq D_{\mathrm{He}}^{R}=g\left(D_{\mathrm{He}}^{G}\right) \leq g\left(D_{\mathrm{He}}^{M}\right) \quad, \quad D_{\mathrm{He}}^{G} \leq(2-\sqrt{2}) D_{\mathrm{He}}^{R} .
$$

Here, the second inequality is established by using $D_{\mathrm{He}}^{R}=g\left(D_{\mathrm{He}}^{G}\right)$ from Table III and $D_{\mathrm{He}}^{M} \leq g\left(D_{\mathrm{He}}^{G}\right)$ from Table I This inequality does not seem to be tight according to Fig. 3 (b). In contrast, the third inequality (and thus also the first inequality) is almost tight for two-qubit states.

Comparison of the discords of response for the Bures, Hellinger, and trace distances. One has

$$
1-\sqrt{1-D_{\mathrm{He}}^{R}} \leq D_{\mathrm{Bu}}^{R} \leq D_{\mathrm{He}}^{R} \leq \sqrt{D_{\mathrm{Tr}}^{R}} \leq \sqrt{2 D_{\mathrm{Bu}}^{R}-\left(D_{\mathrm{Bu}}^{R}\right)^{2}} \leq \sqrt{2 D_{\mathrm{He}}^{R}-\left(D_{\mathrm{He}}^{R}\right)^{2}} .
$$

The first inequality is equivalent to the bound $D_{\mathrm{He}}^{R} \leq 1-\left(1-D_{\mathrm{Bu}}^{R}\right)^{2}$ from Table III It is tight and saturated for pure states (see Theorem 9 below). The other inequalities are trivial consequences of general bounds between the Bures, Hellinger, and trace distance, see Eq. (16). In particular, the last inequality follows from the second one and the monotonicity of $d \mapsto 2 d-d^{2}$ on $[0,1]$.

According to our numerical results in Figs. 1(c) and 1(d), the second and third inequalities in Eq. (27) are almost tight for two qubits. The fourth inequality is saturated for pure states (see Sec. IIIB below). One sees on Fig. 11(d) that the bound $D_{\mathrm{Tr}}^{R} \leq 2 D_{\mathrm{He}}^{R}-\left(D_{\mathrm{He}}^{R}\right)^{2}$ is almost tight. This seems to indicate that there exists a family of two-qubit states saturating the second and fourth inequalities in Eq. (27). Such states cannot be pure since $D_{\mathrm{Bu}}^{R}<D_{\mathrm{He}}^{R}$ for pure states when $D_{\mathrm{Bu}}^{R} \neq 0,1$ (see Table

Comparison of the (measurement-induced) geometric discords for the Bures and Hellinger distances. We find

$$
D_{\mathrm{Bu}}^{G} \leq D_{\mathrm{He}}^{G} \leq g^{-1} \circ h\left(D_{\mathrm{Bu}}^{G}\right) \quad, \quad D_{\mathrm{Bu}}^{M} \leq D_{\mathrm{He}}^{M}
$$

The first and last inequalities are as in Eq. (16). The second one is a consequence of $D_{\mathrm{Bu}}^{R}=g\left(D_{\mathrm{Bu}}^{G}\right), D_{\mathrm{He}}^{R}=g\left(D_{\mathrm{He}}^{G}\right)$ and $D_{\mathrm{He}}^{R} \leq 1-\left(1-D_{\mathrm{Bu}}^{R}\right)^{2}$ from Table III. The latter bound being saturated for pure states (see Theorem 9 below), the same is true for the second inequality, which is tight. Note the similarities between the lower and upper bounds on $D_{\mathrm{Bu}}^{M}$ and $D_{\mathrm{He}}^{G}$ in Eqs. (25) and (28).

According to the numerical results shown in Fig. 2(b), the last inequality on the measurement-induced discords is almost tight for two qubits.

Comparison of the geometric and measurement-induced geometric discords for the trace, Bures, and Hellinger distances. Finally, we obtain a set of inequalities enabling to compare $D^{G}$ and $D^{M}$ :

$$
D_{\operatorname{Tr}}^{G}=D_{\operatorname{Tr}}^{M} \leq h\left(D_{\mathrm{Bu}}^{G}\right) \leq \min \left\{h\left(D_{\mathrm{He}}^{G}\right), h\left(D_{\mathrm{Bu}}^{M}\right)\right\} \leq h\left(D_{\mathrm{He}}^{M}\right) \quad, \quad D_{\mathrm{Bu}}^{G} \leq D_{\mathrm{He}}^{G} \leq(2-\sqrt{2}) \sqrt{D_{\operatorname{Tr}}^{M}} .
$$

The first inequality follows by combining the relations $D_{\operatorname{Tr}}^{G}=D_{\operatorname{Tr}}^{M}=D_{\operatorname{Tr}}^{R}$ and $D_{\mathrm{Bu}}^{R}=g\left(D_{\mathrm{Bu}}^{G}\right)$ with the fourth bound in Eq. (27). As the latter bound, this inequality is saturated for pure states. The second and third inequalities in Eq. (29) are straightforward consequences of the monotonicity of the function $h$ and of the trivial bounds $D_{\mathrm{Bu}}^{G} \leq D_{\mathrm{He}}^{G}$, $D_{\mathrm{Bu}}^{G} \leq D_{\mathrm{Bu}}^{M} \leq D_{\mathrm{He}}^{M}$, and $D_{\mathrm{He}}^{G} \leq D_{\mathrm{He}}^{M}$. The fifth inequality follows from the last bound in Eq. (26) and the third bound in Eq. (27).

The numerical results shown in panels (a) and (c) of Fig. 2 indicate that there exists a family of mixed two-qubit states which nearly saturate the first, second, and third inequalities in Eq. (29), i.e., such that $D_{\operatorname{Tr}}^{M} \simeq h\left(D_{\mathrm{Bu}}^{G}\right) \simeq$ $h\left(D_{\mathrm{He}}^{G}\right) \simeq h\left(D_{\mathrm{Bu}}^{M}\right) \simeq h\left(D_{\mathrm{He}}^{M}\right)$. This provides a numerical hint that these three inequalities could be tight. Moreover, since $h$ is an increasing function, this also tells us that the first bounds in Eqs. (25), (26), and (28) and the last bound in Eq. (28) (trivial bounds) are nearly saturated by this family of two-qubit states. This is in agreement with the conclusions drawn above, namely, the saturation of the first bounds in Eqs. (25) and (26) inferred respectively from Figs. 3(a) and 3(b), and the saturation of the last bound in Eq. (28) inferred from Fig. 2(b). 
(a)

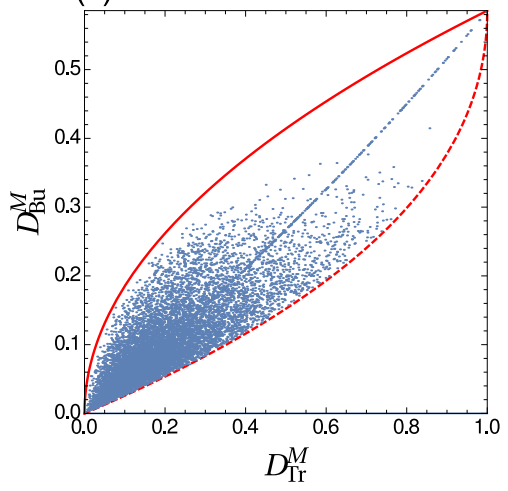

(b)

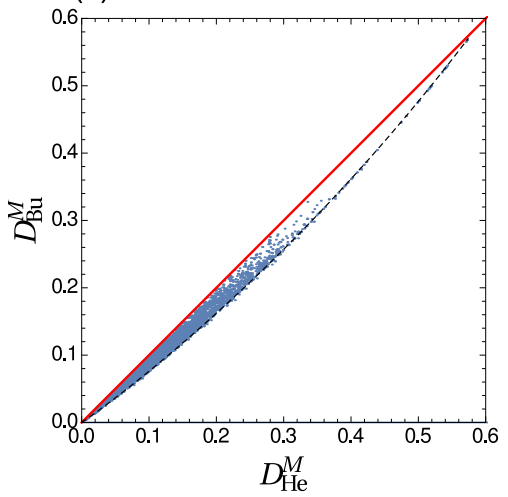

(c)

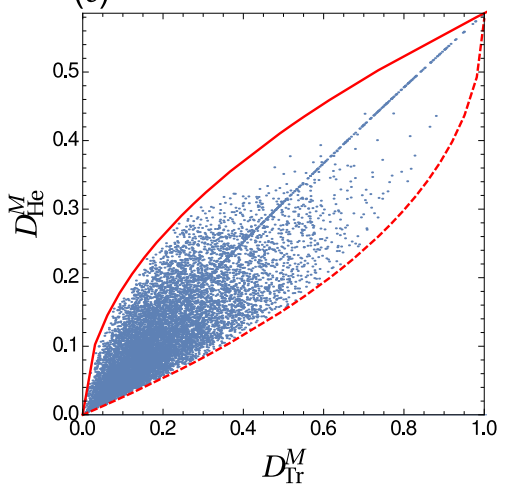

FIG. 2: Comparison of the measurement-induced geometric discords based on the four distances introduced in Sect. [ The points represent $10^{4}$ randomly generated two-qubit states (see main text for details). The lines correspond to equalities in the inequalities of Eqs. (28), (29), and (30). (a) Bures and trace measurement-induced geometric discords $D_{\mathrm{Bu}}^{M}$ and $D_{\mathrm{Tr}}^{M}$. Red solid line: $D_{\mathrm{Bu}}^{M}=(2-\sqrt{2}) \sqrt{D_{\mathrm{Tr}}^{M}}$, see Eqs. (28) and (30); red dashed line: $D_{\mathrm{Bu}}^{M}=h^{-1}\left(D_{\mathrm{Tr}}^{M}\right)$, see Eq. (29). (b) Bures and Hellinger measurement-induced geometric discords $D_{\mathrm{Bu}}^{M}$ and $D_{\mathrm{He}}^{M}$. Red solid line: $D_{\mathrm{Bu}}^{M}=D_{\mathrm{He}}^{M}$, see Eq. (28); blue dashed line: relation $D_{\mathrm{He}}^{M}=2-\left[\left(1+\sqrt{1-g\left(D_{\mathrm{Bu}}^{M}\right)}\right)^{3 / 2}+\left(1-\sqrt{1-g\left(D_{\mathrm{Bu}}^{M}\right)}\right)^{3 / 2}\right] / \sqrt{2}$ satisfied by pure states. (c) Hellinger and trace measurementinduced geometric discords $D_{\mathrm{He}}^{M}$ and $D_{\mathrm{Tr}}^{M}$. Red solid line: $D_{\mathrm{He}}^{M}=(2-\sqrt{2}) \sqrt{D_{\mathrm{Tr}}^{M}}$, corresponding to the conjectured upper bound in Eq. (30); red dashed line: $D_{\mathrm{He}}^{M}=h^{-1}\left(D_{\mathrm{Tr}}^{M}\right)$, see Eq. (29).

(a)

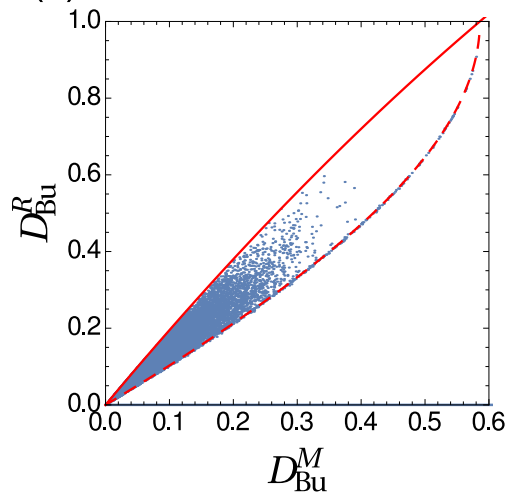

(b)

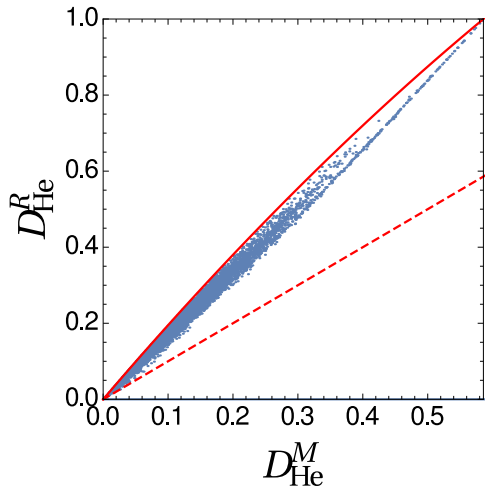

FIG. 3: Comparison of the discords of response and measurement-induced geometric discords based on the same distances. The points represent $10^{4}$ randomly generated two-qubit states (see main text for details). The lines correspond to equalities in the inequalities of Eqs. (25) and (26). (a) Bures discord of response $D_{\mathrm{Bu}}^{R}$ and measurement-induced geometric discord $D_{\mathrm{Bu}}^{M}$. Red solid line: $D_{\mathrm{Bu}}^{R}=g\left(D_{\mathrm{Bu}}^{M}\right)$, see Eq. (25); red dashed line: $D_{\mathrm{Bu}}^{R}=g \circ h^{-1} \circ g\left(D_{\mathrm{Bu}}^{M}\right)=1-\sqrt{1-g\left(D_{\mathrm{Bu}}^{M}\right)}$, corresponding to the saturation achieved for pure states of the second inequality in Eq. (25) with $D_{\mathrm{Bu}}^{G}=g^{-1}\left(D_{\mathrm{Bu}}^{R}\right)$. (b) Hellinger discord of response $D_{\mathrm{He}}^{R}$ and measurement-induced geometric discord $D_{\mathrm{He}}^{M}$. Red solid line: $D_{\mathrm{He}}^{R}=g\left(D_{\mathrm{He}}^{M}\right)$, see Eq. (26); red dashed line: $D_{\mathrm{He}}^{R}=D_{\mathrm{He}}^{M}$, see Eq. (26).

Conjecture. From the result of Fig. 2(c) we conjecture that the following inequality, which is stronger than the fifth inequality in Eq. (29), holds for two-qubit systems:

$$
D_{\mathrm{He}}^{M} \leq(2-\sqrt{2}) \sqrt{D_{\mathrm{Tr}}^{M}}
$$

However, so far, we could not find an analytical proof of Eq. (30).

\section{REVIEW OF PREVIOUS RESULTS}

In this section we review some results already known in the literature that will be used later on in the paper. We begin by recalling the definitions of the entropic quantum discord and the local quantum uncertainty (see Sec. IIIA). 
Next, we discuss some important properties of the four distances of interest on the set of quantum states, in particular some bounds between them (see Sec. IIIB). Finally, we summarize the main arguments showing that $D^{G}$, $D^{M}$, and $D^{R}$ are bona fide measures of quantum correlations (see Sec. IIIC) and briefly review the results of Ref. 9] on the Bures geometric discord (see Sec. IIID).

\section{A. The entropy-based quantum discord and local quantum uncertainty}

We recall in this subsection the definitions of the entropic quantum discord introduced by Ollivier and Zurek 1, 2] and by Henderson and Vedral [3] and of the LQU introduced by Girolami, Tufarelli, and Adesso [31].

Consider a bipartite quantum system composed of subsystems $A$ and $B$, in the state $\varrho \equiv \varrho_{A B}$. The total correlations between the two parties are characterized by the mutual information

$$
I_{A: B}(\varrho) \equiv S\left(\varrho_{B}\right)+S\left(\varrho_{A}\right)-S(\varrho),
$$

where the information (ignorance) about the state of $A B$ is given by the von Neumann entropy $S(\varrho) \equiv-\operatorname{Tr} \varrho \log \varrho$, and similarly for subsystems $A$ and $B$. In classical information theory, the mutual information is equal to the difference between the Shannon entropy of $B$ and the conditional entropy of $B$ conditioned on $A$. In the quantum setting, the corresponding quantity is

$$
J_{A: B}^{\left\{\Pi_{i}^{A}\right\}}(\varrho) \equiv S\left(\varrho_{B}\right)-\sum_{i} p_{i} S\left(\varrho_{B \mid i}\right)
$$

where $p_{i}=\operatorname{Tr}\left(\varrho \Pi_{i}^{A} \otimes \mathbb{1}\right)$ is the probability of outcome $i$ in a local von Neumann measurement on $A$ defined by the orthogonal projectors $\Pi_{i}^{A}$, and $\varrho_{B \mid i}=p_{i}^{-1} \operatorname{Tr}_{A}\left(\varrho \Pi_{i}^{A} \otimes \mathbb{1}\right)$ is the corresponding conditional post-measurement state of $B$. It turns out that $I_{A: B}$ and $J_{A: B}$ are in general not equal for quantum systems. Moreover, $I_{A: B}$ is never smaller than $J_{A: B}$, whatever the local measurement. Therefore, one can define the entropic quantum discord as [1 3

$$
D_{A}^{\mathrm{ent}}(\varrho) \equiv I_{A: B}(\varrho)-\max _{\left\{\Pi_{i}^{A}\right\}} J_{A: B}^{\left\{\Pi_{i}^{A}\right\}}(\varrho) \geq 0
$$

This quantity is interpreted as a quantifier of the non-classical features of the bipartite state $\varrho$. Note that it is not symmetric under the exchange of the two parties. One defines the quantum discord $D_{B}^{\text {ent }}(\varrho)$ analogously, by considering local measurements on subsystem $B$. The two entropic discords $D_{A}^{\text {ent }}$ and $D_{B}^{\text {ent }}$ give the amount of bipartite mutual information that cannot be retrieved by measuring one of the subsystems. Actually, one has

$$
D_{A}^{\mathrm{ent}}(\varrho)=\min _{\left\{\Pi_{i}^{A}\right\}}\left\{I_{A: B}(\varrho)-I_{A: B}\left(\varrho_{\mathrm{p} . \mathrm{m} .}^{\left\{\Pi_{i}^{A}\right\}}\right)\right\}
$$

where $\varrho_{\mathrm{p} . \mathrm{i}}^{\left\{\Pi_{i}^{A}\right\}}$ is the post-measurement state after a local measurement on $A$ with rank-one orthogonal projectors $\Pi_{i}^{A}$, see Eq. (6), and the minimum is taken over the set of all such measurements.

It can be shown that the entropic discord $D_{A}^{\text {ent }}$ obeys Axioms (i-v) stated in the Introduction, so that it is a proper measure of quantum correlations (see Ref. [15] for a thorough review). For instance, condition (iv) is fulfilled since $D_{A}^{\text {ent }}$ coincides for pure states with the entanglement of formation, an entanglement monotone that reduces on pure states to the von Neumann entropy of the reduced states [38]. For mixed states, the entropic discord captures quantum correlations different from entanglement. Indeed, most separable (i.e., unentangled) mixed states have non-vanishing discords. Moreover, a nonzero discord might be responsible for the improvement (quantum speed-up) of certain quantum algorithms with respect to their classical analogs [39], although this claim is still debated (see e.g. the survey article in Ref. [5]). On the other hand, the analytical evaluation of the entropic discord remains a formidably challenging task, even for two-qubit states, because of the difficulty of the optimization problem over the quantum measurements. In this respect, distance-based measures of quantum correlations are usually less challenging to evaluate, as it has been illustrated in Sec. IIC Moreover, they often have operational interpretations in terms of state and channel discrimination.

A different measure of quantum correlations called the local quantum uncertainty (LQU) was introduced in Ref. [31]. It is defined as follows. One says that a quantum observable is measured in a given state $\varrho$ without quantum uncertainty if the measurement does not disturb $\varrho$. The degree of perturbation is quantified by the skew information [40, 41]

$$
\mathcal{L}(\varrho, K) \equiv-\frac{1}{2} \operatorname{Tr}\left([\sqrt{\varrho}, K]^{2}\right)
$$

where $K$ is the measured observable and $[X, Y] \equiv X Y-Y X$ denotes the commutator. The skew information is considered a proper measure of the measurement uncertainty because of the following properties: it is non-negative, 
it vanishes if and only if the operators commute, and it is convex in $\varrho$. Moreover, it is bounded by the variance of the measured observable: $\mathcal{L}(\varrho, K) \leq \operatorname{Tr} K^{2} \varrho-(\operatorname{Tr} K \varrho)^{2}$, with equality holding for pure states [41] (see also Ref. [42]). In particular, in protocols of parameter estimation the skew information is related to the quantum Fisher information and to the Cramér-Rao bound [29, 41]. Applying the skew information to local observables $K_{A}^{\Lambda}$ acting on subsystem $A$ with spectrum $\Lambda$ and minimizing over all such self-adjoint operators, one defines the LQU as [31]:

$$
\mathcal{U}_{A}^{\Lambda}(\varrho) \equiv \min _{K_{A}^{\Lambda}} \mathcal{L}\left(\varrho, K_{A}^{\Lambda} \otimes \mathbb{1}\right)
$$

where the spectrum $\Lambda$ is fixed and non-degenerate in order to exclude the identity operator. If $A$ is a qubit then the dependence on $\Lambda$ of $\mathcal{U}_{A}^{\Lambda}(\varrho)$ reduces to a multiplication by a constant factor [31]. Therefore, one can restrict the definition of the LQU to the case where $\Lambda$ is the harmonic spectrum: $\Lambda=\{1,-1\}$. All Hermitian $2 \times 2$ matrices with harmonic spectrum are unitary matrices. It follows from this observation that if $A$ is a qubit then the LQU is equal to the Hellinger discord of response $D_{\mathrm{He}}^{R}$.

The LQU was evaluated explicitly for arbitrary qubit-qudit states in Ref. 31]. It was found in this reference that $1-\mathcal{U}_{A}^{\{1,-1\}}(\varrho)=\lambda_{\max }(W)$ is the highest eigenvalue of the $3 \times 3$ matrix with elements $W_{i j}=\operatorname{Tr} \sqrt{\varrho} \sigma_{i} \otimes \mathbb{1} \sqrt{\varrho} \sigma_{j} \otimes \mathbb{1}$. A simple calculation shows that $\mathcal{U}_{A}^{\{1,-1\}}(\varrho)$ is then given by the right-hand side of Eq. (22).

\section{B. Properties and comparison of the trace, Hilbert-Schmidt, Bures, and Hellinger distances}

In this subsection we recall some known facts about the four distances defined in Eqs. (2)-(4). In quantum information theory, well-behaved distances $d$ on the set of quantum states must be contractive under Completely Positive Trace Preserving (CPTP) maps, that is, they must be such that $d(\Phi(\varrho), \Phi(\sigma)) \leq d(\varrho, \sigma)$ for any states $\varrho$ and $\sigma$ and any CPTP map $\Phi$ acting on the algebra of operators from the system Hilbert space $\mathcal{H}$ to a (possibly different) space $\mathcal{H}^{\prime}$ (see Sec. VIII for a definition of CPTP maps) [15, 43]. Notice also that a contractive distance $d$ is in particular unitarily invariant (i.e., $d\left(U \varrho U^{\dagger}, U \sigma U^{\dagger}\right)=d(\varrho, \sigma)$ for any unitary operator $\left.U\right)$.

Let us first focus on the trace and Hilbert-Schmidt distances $d_{\mathrm{Tr}}$ and $d_{\mathrm{HS}}$. The former is contractive under CPTP maps, but this is not the case for the latter (more generally, as already mentioned, the distances associated to the $p$-norms $\|X\|_{p}=\left(\operatorname{Tr}|X|^{p}\right)^{1 / p}$ are not contractive for $\left.p>1\right)$ [15, 35, 37, 43]. The trace distance achieves an operational meaning in the light of the Helstrom formula $P_{\operatorname{err}}(\varrho, \sigma)=1 / 2-d_{\operatorname{Tr}}(\varrho, \sigma) / 4$ for the minimum probability of error in discriminating two equiprobable quantum states $\varrho$ and $\sigma$. The Hilbert-Schmidt distance can be used to bound from above and below the trace distance as follows:

$$
\frac{1}{\sqrt{r}} d_{\operatorname{Tr}}(\varrho, \sigma) \leq d_{\mathrm{HS}}(\varrho, \sigma) \leq d_{\operatorname{Tr}}(\varrho, \sigma)
$$

where $r$ is the rank of $\varrho-\sigma$. The inequalities on the geometric discords given in Eq. (15) are trivial consequences of Eq. (37).

The Bures distance $d_{\mathrm{Bu}}$ defined in Eq. (3) coincides with the Fubini-Study distance for pure states. It is Riemannian and contractive under CPTP maps. In fact, it is the smallest distance featuring these two properties [44] (see also Ref. [15]). It can be bounded in terms of the trace distance and vice versa thanks to the following inequalities [43, 45]:

$$
d_{\mathrm{Bu}}(\varrho, \sigma)^{2} \leq d_{\operatorname{Tr}}(\varrho, \sigma) \leq \sqrt{2 g\left(d_{\mathrm{Bu}}(\varrho, \sigma)^{2}\right)}
$$

where $g$ is the function given in Eq. (23).

The (quantum) Hellinger distance $d_{\mathrm{He}}$ defined in Eq. (4) appears naturally in the context of the quantum Chernoff bound [46]. For commuting states, it reduces, just like the Bures distance, to the (classical) Hellinger distance between probability distributions. Although its definition bears some similarity with that of the Hilbert-Schmidt distance, it shares many properties of the Bures distance. Indeed, $d_{\mathrm{He}}$ is Riemannian and contractive with respect to CPTP maps (the contractivity can be derived from Lieb's concavity theorem [47], see Ref. [46]). Since $d_{\mathrm{Bu}}$ is the smallest Riemannian contractive distance, for any states $\varrho$ and $\sigma$ one has

$$
d_{\mathrm{Bu}}(\varrho, \sigma) \leq d_{\mathrm{He}}(\varrho, \sigma)
$$

Remarkably, there exists also an important bound of the trace distance in terms of the Hellinger distance [48]:

$$
d_{\mathrm{He}}(\varrho, \sigma)^{2} \leq d_{\operatorname{Tr}}(\varrho, \sigma) \leq \sqrt{2 g\left(d_{\mathrm{He}}(\varrho, \sigma)^{2}\right)} .
$$

The first, second, and third inequalities on the geometric discords in Eq. (16) are trivial consequences of Eqs. (39), (40), and (38), respectively. The corresponding bounds for the discord of response, which are obtained by taking into account the normalization factor in Eq. (7), read

$$
D_{\mathrm{Bu}}^{R}(\varrho)^{2} \leq D_{\mathrm{He}}^{R}(\varrho)^{2} \leq D_{\operatorname{Tr}}^{R}(\varrho) \leq 1-\left(1-D_{\mathrm{Bu}}^{R}(\varrho)\right)^{2} .
$$


The last inequality holds as an equality for pure states. Indeed, the upper bound on the trace distance in Eq. (38) is saturated when both $\varrho$ and $\sigma$ are pure [43]. This explains why the fourth bound in Eq. (27) and the first bound in Eq. (29) are tight and saturated for pure two-qubit states. Our numerical results displayed in Fig. 11(c) indicate that the first bound in Eq. (41) is almost tight when $n_{A}=n_{B}=2$.

Let us also point out that the Bures and Hellinger distances are monotonic under tensor products, that is, if two states $\varrho_{1}$ and $\sigma_{1}$ are closer to each other than two other states $\varrho_{2}$ and $\sigma_{2}$, then the same is true for the states $\varrho_{1}^{\otimes 2}$, $\sigma_{1}^{\otimes 2}$ and $\varrho_{2}^{\otimes 2}, \sigma_{2}^{\otimes 2}$, when considering two identical copies of the system. We remark that the trace distance does not enjoy this property.

\section{Geometric measures as proper measures of quantum correlations}

By using known results in the literature, we show in this subsection that $D^{G}, D^{M}$, and $D^{R}$ are bona fide measures of quantum correlations satisfying Axioms (i-iv) of Section $\$ when the distance is the trace, Bures, or Hellinger distance.

Let us first prove that $D^{G}, D^{M}$, and $D^{R}$ satisfy Axiom (i), irrespective of the choice of the distance. This is obvious for the geometric discord. For the measurement-induced geometric discord, this comes from the fact that a state is classical-quantum if and only if it is invariant under a von Neumann measurement on $A$ with rank-one projectors [2, 15]. Note that this would not be true if the minimization in Eq. (6) was performed over projectors $\Pi_{i}^{A}$ with ranks larger than one. For the discord of response, the validity of Axiom (i) is a consequence of the following result derived in Ref. [11]: the only bipartite states for which there exists a local unitary transformation on $A$ such that the unitary operator has a non-degenerate spectrum and the state is invariant under the transformation are the classical-quantum states.

The fact that the three geometric measures obey Axiom (ii) holds for any unitary invariant distance, and thus in particular for the trace, Hilbert-Schmidt, Bures, and Hellinger distances.

It is easy to verify that $D^{G}, D^{M}$, and $D^{R}$ satisfy the monotonicity Axiom (iii) provided that $d$ is contractive under CPTP maps (see, e.g., Ref. [15]). As explained above, this is the case for the trace, Bures, and Hellinger distances, but not for the Hilbert-Schmidt distance.

It remains to prove that the geometric measures satisfy Axiom (iv), i.e., that they reduce to entanglement monotones for pure states. To this end, one can exploit the following known results:

(a) For pure states, the Bures discord of response is given by [11]:

$$
D_{\mathrm{Bu}}^{R}(|\Psi\rangle)=1-\left(1-E^{R}(|\Psi\rangle)\right)^{\frac{1}{2}},
$$

where $E^{R}(|\Psi\rangle)$ is the entanglement of response [49] (or unitary entanglement), originally introduced in Ref. [50], which is equal to one minus the maximum fidelity between the pure state $|\Psi\rangle$ and the state obtained by perturbing $|\Psi\rangle$ with a local unitary operator $U_{A} \in \mathcal{U}_{\Lambda}$ :

$$
E^{R}(|\Psi\rangle) \equiv \min _{U_{A} \in \mathcal{U}_{\Lambda}}\left\{1-\left|\left\langle\Psi\left|U_{A} \otimes \mathbb{1}\right| \Psi\right\rangle\right|^{2}\right\}
$$

The entanglement of response is an entanglement monotone and can be extended to mixed states via the convex roof construction [49].

(b) The trace and Hilbert-Schmidt discords of response $D_{\mathrm{Tr}}^{R}$ and $D_{\mathrm{HS}}^{R}$ coincide exactly with the entanglement of response $E^{R}$ on pure states.

(c) The Bures geometric discord $D_{\mathrm{Bu}}^{G}$ reduces for pure states to [9, 15]:

$$
D_{\mathrm{Bu}}^{G}(|\Psi\rangle)=2-2\left(1-E^{W}(|\Psi\rangle)\right)^{\frac{1}{2}},
$$

where $E^{W}(|\Psi\rangle) \equiv \min \left\{1-\left|\left\langle\Phi_{\mathrm{S}} \mid \Psi\right\rangle\right|^{2}\right\}$ is the Wei-Goldbart measure of global geometric entanglement [51]. Here, the minimum is taken over all separable pure states $\left|\Phi_{\mathrm{s}}\right\rangle$ (i.e., product states). The measure $E^{W}$ is an entanglement monotone (see e.g. Ref. [15]). It has been extended to pure multipartite hierarchies in Ref. [52] and to mixed bipartite states in Ref. [53].

(d) Let $\delta(\varrho, \sigma)$ be a non-negative function defined on the set of pairs of quantum states, which is contractive with respect to CPTP maps and satisfies the 'flags' condition $\delta\left(\sum_{i} p_{i} \varrho_{i} \otimes|i\rangle\left\langle i\left|, \sum_{i} p_{i} \sigma_{i} \otimes\right| i\right\rangle\langle i|\right)=\sum_{i} p_{i} \delta\left(\varrho_{i}, \sigma_{i}\right)$. Let $\mathcal{L}_{A}$ be a family of CPTP maps on subsystem $A$ which is closed under unitary conjugations. It is proven in Ref. [16] that the measure of quantumness $Q_{\delta, \mathcal{L}_{A}}$ defined by

$$
Q_{\delta, \mathcal{L}_{A}}(\varrho)=\inf _{\Lambda_{A} \in \mathcal{L}_{A}} \delta\left(\varrho, \Lambda_{A} \otimes 1(\varrho)\right)
$$

is entanglement monotone when restricted to pure states. 
Exploiting statements (a-b) above and Eq. (17), it follows that on pure states

$$
D_{\mathrm{He}}^{R}(|\Psi\rangle)=D_{\mathrm{HS}}^{R}(|\Psi\rangle)=D_{\operatorname{Tr}}^{R}(|\Psi\rangle)=1-\left(1-D_{\mathrm{Bu}}^{R}(|\Psi\rangle)\right)^{2}=E^{R}(|\Psi\rangle) .
$$

Therefore, the discord of response satisfies Axiom (iv) for the trace, Hilbert-Schmidt, Bures, and Hellinger distances.

Note that in this paper we call entanglement monotone any measure $E$ on pure states such that $E(|\Psi\rangle) \geq E(|\Phi\rangle)$ whenever $|\Phi\rangle$ can be obtained from $|\Psi\rangle$ by local operations and classical communication between the two parties $A$ and $B$. We do not ask here that $E$ obeys the stronger monotonicity requirement $E(|\Psi\rangle) \geq \sum_{i} p_{i} E\left(\left|\Phi_{i}\right\rangle\right)$, where the right-hand side is the average entanglement of the post-selected state $\left|\Phi_{i}\right\rangle$ conditioned to the measurement outcome $i$ and $p_{i}$ is the corresponding probability, the inequality being true for arbitrary measurements (including non-projective ones). Hence, according to our definition, if $E$ is an entanglement monotone and $f$ is a non-decreasing function (not necessarily concave), then $f(E)$ is still an entanglement monotone. Given that the entanglement of response $E^{R}$ is strongly entanglement monotone [49], by Eq. (46) the discords of response $D_{\mathrm{He}}^{R}, D_{\mathrm{HS}}^{R}$, and $D_{\mathrm{Tr}}^{R}$ actually satisfy a stronger version of Axiom (iv) in which entanglement monotone is replaced by strongly entanglement monotone. This statement is also true for the Bures discord of response $D_{\mathrm{Bu}}^{R}$, as it can be shown by using Eqs. (43) and (44) and the characterization of strongly entanglement monotones in Ref. [54].

By the property (c) above, the Bures geometric discord $D_{\mathrm{Bu}}^{G}$ satisfies Axiom (iv). Later on, we will show that this is also the case for the Hellinger geometric discord $D_{\mathrm{He}}^{G}$ (see Section IV] and for the Bures and Hellinger measurementinduced geometric discords $D_{\mathrm{Bu}}^{M}$ and $D_{\mathrm{He}}^{M}$ (see Section $\mathrm{V}$ ). Note that the right-hand side of Eq. (44) is not strongly entanglement monotone [15], so that $D_{\mathrm{Bu}}^{G}$ does not satisfy the aforementioned stronger version of Axiom (iv).

The general property $(\mathrm{d})$ implies that the measurement-induced geometric discord $D^{M}$ and the discord of response $D^{R}$ fulfill Axiom (iv) for the trace, Bures, and Hellinger distances. Indeed, one easily checks that the trace distance and the square Bures and Hellinger distances obey the 'flags' condition. By taking $\mathcal{L}_{A}$ to be the family of rankone projective measurement CPTP maps $\varrho \mapsto \varrho_{\mathrm{p} . \mathrm{m} .}^{\left\{\Pi_{i}^{A}\right\}}$ on $A$ defined by Eq. [6] , one finds that $D^{M}$ is entanglement monotone on pure states for the trace, Bures, and Hellinger distances. The same statement holds for $D^{R}$ and is obtained by taking $\mathcal{L}_{A}$ to be the family of local unitary conjugations $\varrho \mapsto U_{A} \otimes \mathbb{1} \varrho U_{A}^{\dagger} \otimes \mathbb{1}$ for all unitary operators $U_{A}$ with spectrum $\Lambda$. Actually, one can deduce from Theorem 2.4 of Ref. [16] that $\left(D_{\mathrm{Tr}}^{M}\right)^{1 / 2}, D_{\mathrm{Bu}}^{M}$ and $D_{\mathrm{He}}^{M}$ satisfy the strong version of Axiom (iv), and similarly for $\left(D_{\mathrm{Tr}}^{R}\right)^{1 / 2}, D_{\mathrm{Bu}}^{R}$, and $D_{\mathrm{He}}^{R}$, in agreement with our observation above.

Finally, the trace geometric discord $D_{T r}^{G}$ obeys Axiom (iv) at least when subsystem $A$ is a qubit, because in this case one has $D_{\operatorname{Tr}}^{G}=D_{\operatorname{Tr}}^{M}=D_{\operatorname{Tr}}^{R}$, see Eq. (24).

In summary, for the trace, Bures, and Hellinger distances, $D^{G}, D^{M}$, and $D^{R}$ are all bona fide measures of quantum correlations whatever the dimensionality $n_{A}$ of subsystem $A$ (except perhaps for $D_{\mathrm{Tr}}^{G}$, for which we are not aware of a proof of Axiom (iv) in the case $n_{A}>2$ ). In contrast, if one chooses the Hilbert-Schmidt distance, then $D^{G}, D^{M}$, and $D^{R}$ are not contractive under local CPTP maps acting on subsystem $B$ and are thus not reliable measures of quantum correlations. This comes from the lack of monotonicity of the Hilbert-Schmidt distance already mentioned several times in this paper. Indeed, an explicit counter-example showing that $D_{\mathrm{HS}}^{G}=D_{\mathrm{HS}}^{M}$ does not satisfy Axiom (iii) has been constructed in Ref. [34]. It is enough to consider the quantum operation which consists in tracing out over an ancilla $C$, i.e., the CPTP map $\Phi_{B}(\varrho)=\operatorname{Tr}_{C}(\varrho)$. Here, the subsystem $B$ consists of two parts $B^{\prime}$ and $C$ (that is, $C$ is included in $B$ ). If the total system $A B$ is in state $\varrho=\varrho_{A B^{\prime}} \otimes \varrho_{C}$ with no correlations between $A B^{\prime}$ and $C$, adding or removing the ancilla $C$ cannot affect the quantum correlations between $A$ and $B$. However, due to the property $d_{\mathrm{HS}}\left(\varrho_{A B^{\prime}} \otimes \varrho_{C}, \sigma_{A B^{\prime}} \otimes \varrho_{C}\right)=d_{\mathrm{HS}}\left(\varrho_{A B^{\prime}}, \sigma_{A B^{\prime}}\right)\left\|\varrho_{C}\right\|_{\mathrm{HS}}$, one finds

$$
D_{\mathrm{HS}}^{M}(\varrho)=D_{\mathrm{HS}}^{M}\left(\Phi_{B}(\varrho)\right) \operatorname{Tr}\left(\varrho_{C}^{2}\right) .
$$

If the ancilla $C$ is not in a pure state then $\operatorname{Tr}\left(\varrho_{C}^{2}\right)<1$ and thus $D_{\mathrm{HS}}^{M}\left(\Phi_{B}(\varrho)\right)>D_{\mathrm{HS}}^{M}(\varrho)$. By the same argument, $D_{\mathrm{HS}}^{R}\left(\Phi_{B}(\varrho)\right)>D_{\mathrm{HS}}^{R}(\varrho)$. Therefore, $D_{\mathrm{HS}}^{G}=D_{\mathrm{HS}}^{M}$ and $D_{\mathrm{HS}}^{R}$ are not bona fide measures of quantum correlations.

\section{Bures geometric discord and quantum state discrimination}

We describe in this subsection the operational interpretation of the Bures geometric discord $D_{\mathrm{Bu}}^{G}$ and introduce the closest classical-quantum states of a given state $\varrho$ for the Bures distance in terms of a quantum state discrimination problem. We briefly review the results of Ref. [9], which justify Eq. (12) and will be used several times in what follows.

Let us first introduce the maximum probability of success in discriminating the states $\varrho_{i}$ with prior probabilities $\eta_{i}$ by means of projective measurements on $A B$ of rank $n_{B}$, which is given by

$$
P_{\mathrm{S}}^{\text {optv.N. }}\left(\left\{\varrho_{i}, \eta_{i}\right\}\right)=\max _{\left\{\Pi_{i}\right\}} \sum_{i=1}^{n_{A}} \eta_{i} \operatorname{Tr}\left(\Pi_{i} \varrho_{i}\right),
$$

the maximum being taken over all families $\left\{\Pi_{i}\right\}$ of orthogonal projectors on $\mathcal{H}_{A} \otimes \mathcal{H}_{B}$ with rank $n_{B}$. Consider now that the Bures geometric discord is expressed in terms of the maximum fidelity between $\varrho$ and a classical-quantum 
state, $F(\varrho, \mathcal{C Q}) \equiv \max _{\sigma_{\mathrm{cq}} \in \mathcal{C} \mathcal{Q}} F\left(\varrho, \sigma_{\mathrm{cq}}\right)$, by (see Eqs. (3) and (15) ):

$$
D_{\mathrm{Bu}}^{G}(\varrho)=2-2 \sqrt{F(\varrho, \mathcal{C Q})} .
$$

The fidelity $F(\varrho, \mathcal{C Q})$ is equal to $[9,15]$ :

$$
F(\varrho, \mathcal{C} \mathcal{Q})=\max _{\left\{\left|\alpha_{i}\right\rangle\right\}} P_{\mathrm{S}}^{\text {optv.N. }}\left(\left\{\varrho_{i}, \eta_{i}\right\}\right),
$$

where the states $\varrho_{i}$ to be discriminated and their probabilities $\eta_{i}$ depend on the orthonormal basis $\left\{\left|\alpha_{i}\right\rangle\right\}$ for $A$ and are given by Eq. (11), and the maximum is taken over all such bases. If the density matrix $\varrho$ is invertible, the optimal measurement in ambiguous quantum state discrimination to discriminate the $\varrho_{i}$ 's is a von Neumann measurement with projectors of rank $n_{B}$. Hence $F(\varrho, \mathcal{C Q})$ is also equal to the maximum over all orthonormal bases $\left\{\left|\alpha_{i}\right\rangle\right\}$ of the optimal success probability $P_{\mathrm{S}}^{\mathrm{opt}}\left(\left\{\varrho_{i}, \eta_{i}\right\}\right)$ to discriminate the states $\varrho_{i}$ using arbitrary POVMs.

For the Bures distance, the classical-quantum states $\sigma_{\mathrm{Bu}, \varrho}$ closest to $\varrho$ are expressed in terms of the optimal basis vectors $\left|\alpha_{i}^{\mathrm{opt}}\right\rangle$ and optimal orthogonal projectors $\Pi_{i}^{\mathrm{opt}}$ maximizing the right-hand sides of Eqs. (50) and (48):

$$
\sigma_{\mathrm{Bu}, \varrho}=\frac{1}{F(\varrho, \mathcal{C} \mathcal{Q})} \sum_{i=1}^{n_{A}}\left|\alpha_{i}^{\mathrm{opt}}\right\rangle\left\langle\alpha_{i}^{\mathrm{opt}}\right| \otimes\left\langle\alpha_{i}^{\mathrm{opt}}\left|\sqrt{\varrho} \Pi_{i}^{\mathrm{opt}} \sqrt{\varrho}\right| \alpha_{i}^{\mathrm{opt}}\right\rangle .
$$

When $A$ is a qubit, one has to discriminate $n_{A}=2$ states. It is then a simple task to evaluate the maximum success probability. The latter is given by the celebrated Helstrom formula [55] (see also Ref. [15], Corollary 11.B.6):

$$
P_{\mathrm{S}}^{\text {optv.N. }}\left(\left\{\varrho_{i}, \eta_{i}\right\}\right)=\frac{1}{2}\left(1+\left\|\eta_{2} \varrho_{2}-\eta_{1} \varrho_{1}\right\|_{\mathrm{Tr}}\right) .
$$

With the help of Eq. (50), one can show that $D_{\mathrm{Bu}}^{G}$ satisfies the last Axiom (v) of Section प, in addition to the other Axioms (i-iv) [9, 15]. When $n_{A} \leq n_{B}$, the maximum value of $D_{\mathrm{Bu}}^{G}$ is $D_{\max }=2-2 / \sqrt{n_{A}}$, as reported in Table【.

The bounds on $D_{\mathrm{Bu}}^{G}$ reported in Tables $\Pi$ and $\amalg$ will be derived in Sections IV and $\mathrm{I}$ by combining Eq. (50) with some known bounds on the maximum success probability in quantum state discrimination theory.

\section{HELLINGER GEOMETRIC DISCORD: COMPUTABLE AND BONA FIDE MEASURE OF QUANTUM CORRELATIONS}

Geometric discords have been studied in Refs. [14, 21, 56] for the trace distance and in Refs. [8 10, 22, 23] for the Bures distance. In contrast, to the best of our knowledge, the Hellinger geometric discord has not been studied in previous works for finite-dimensional systems.

For two-mode Gaussian states $\varrho$ of a continuous-variable system, the Gaussian geometric discord, defined as the minimal Hellinger distance between $\varrho$ and a classical-quantum Gaussian state, has been investigated in Ref. [57]. However, since, quite remarkably, for Gaussian states the classical-quantum states coincide with product states, this Gaussian discord is actually a measure of total (classical plus quantum) rather than quantum correlations. Thus it only provides an upper bound on the Hellinger geometric discord $D_{\mathrm{He}}^{G} \varrho$ ) for Gaussian states $\varrho$ (in fact, the closest classical-quantum state to $\varrho$ is not necessarily Gaussian). A general study that analyzes and compares geometric and entropic measures of quantum correlations for Gaussian states is in preparation and will appear in a forthcoming paper [58].

In this section, we show that if the Hilbert spaces of $A$ and $B$ have finite dimensions, for any pure state $|\Psi\rangle$, $D_{\mathrm{He}}^{G}(|\Psi\rangle)$ is a simple function of the Schmidt number of $|\Psi\rangle$ and for any mixed state $\varrho, D_{\mathrm{He}}^{G}(\varrho)$ is simply related to the Hilbert-Schmidt geometric discord $D_{\mathrm{HS}}^{G}$ of the square root of $\varrho$. One deduces from the first statement that $D_{\mathrm{He}}^{G}$ enjoys all the properties (i-iv) of bona fide measures of quantum correlations, in contrast to the Hilbert-Schmidt geometric discord (see Sec. IIIC). Given the computability of $D_{\mathrm{HS}}^{G}$, it follows from the second statement that $D_{\mathrm{He}}^{G}$ is also easy to compute, as confirmed by the closed formula for qubit-qudit states derived in Sec. IIC. We also determine the closest classical-quantum state of a given state $\varrho$ with respect to the Hellinger distance. Finally, we show that the Hellinger geometric discord provides upper and lower bounds on the Bures geometric discord.

\section{A. General expressions and closest classical-quantum states}

Recall that the Schmidt number of a pure state $|\Psi\rangle$ of a bipartite system $A B$ is defined as $K(|\Psi\rangle)=\left(\sum_{i} \mu_{i}^{2}\right)^{-1}$, where $\mu_{i}$ are the eigenvalues of the reduced state $[\varrho \Psi]_{A}=\operatorname{Tr}_{B}|\Psi\rangle\langle\Psi|$, that is, the non-negative coefficients appearing in the Schmidt decomposition

$$
|\Psi\rangle=\sum_{i=1}^{n} \sqrt{\mu_{i}}\left|\varphi_{i}\right\rangle\left|\chi_{i}\right\rangle
$$


Here, $n=\min \left\{n_{A}, n_{B}\right\}$ and $\left\{\left|\varphi_{i}\right\rangle\right\}_{i=1}^{n_{A}}$ (respectively $\left\{\left|\chi_{j}\right\rangle\right\}_{j=1}^{n_{B}}$ ) is an orthonormal basis for $A$ (for $B$ ).

Theorem 1. (a) If $|\Psi\rangle$ is a pure state of $A B$, then

$$
D_{\mathrm{He}}^{G}(|\Psi\rangle)=2-2 K(|\Psi\rangle)^{-\frac{1}{2}}
$$

where $K(|\Psi\rangle)$ is the Schmidt number of $|\Psi\rangle$. Furthermore, the closest classical-quantum state to $|\Psi\rangle$ for the Hellinger distance is the classical-classical state

$$
\sigma_{\mathrm{He}, \Psi}=K(|\Psi\rangle) \sum_{i=1}^{n} \mu_{i}^{2}\left|\varphi_{i}\right\rangle\left\langle\varphi_{i}|\otimes| \chi_{i}\right\rangle\left\langle\chi_{i}\right|
$$

(b) If $\varrho$ is a mixed state of $A B$, then

$$
D_{\mathrm{He}}^{G}(\varrho)=2-2 \max _{\left\{\left|\alpha_{i}\right\rangle\right\}}\left\{\sum_{i=1}^{n_{A}} \operatorname{Tr}_{B}\left[\left\langle\alpha_{i}|\sqrt{\varrho}| \alpha_{i}\right\rangle^{2}\right]\right\}^{\frac{1}{2}}
$$

where the maximum is over all orthonormal bases $\left\{\left|\alpha_{i}\right\rangle\right\}$ for $A$. Let this maximum be reached for the basis $\left\{\left|\alpha_{i}^{\mathrm{opt}}\right\rangle\right\}$. Then the closest classical-quantum state to $\varrho$ for the Hellinger distance is

$$
\sigma_{\mathrm{He}, \varrho}=\left(1-\frac{D_{\mathrm{He}}^{G}(\varrho)}{2}\right)^{-2} \sum_{i=1}^{n_{A}}\left|\alpha_{i}^{\mathrm{opt}}\right\rangle\left\langle\alpha_{i}^{\mathrm{opt}}\right| \otimes\left\langle\alpha_{i}^{\mathrm{opt}}|\sqrt{\varrho}| \alpha_{i}^{\mathrm{opt}}\right\rangle^{2} .
$$

Since $K(|\Psi\rangle)$ is an entanglement monotone, one infers from Eq. (54) that $D_{\mathrm{He}}^{G}$ satisfies Axiom (iv) of Section 1 . Moreover, it also satisfies the remaining Axioms (i-iii) because the Hellinger distance is contractive under CPTP maps (see Sec. IIIC). Therefore one has:

Corollary 1. The Hellinger geometric discord $D_{\mathrm{He}}^{G}$ is a bona fide measure of quantum correlations. Its maximum value is given by $D_{\max }=2-2 / \sqrt{n_{A}}$ when $n_{A} \leq n_{B}$.

The last statement follows from Eq. (54) and the fact that if $n_{A} \leq n_{B}$ then $D_{\mathrm{He}}^{G}$ is maximum for maximally entangled pure states (see the Introduction).

It is enlightening to compare the results of Theorem 1 to the corresponding results for the Bures distance. For pure states, one has (see Table [and Ref. [9])

$$
D_{\mathrm{Bu}}^{G}(|\Psi\rangle)=2\left(1-\sqrt{\mu_{\max }}\right)
$$

and

$$
\sigma_{\mathrm{Bu}, \Psi}=\left|\varphi_{\max }\right\rangle\left\langle\varphi_{\max }|\otimes| \chi_{\max }\right\rangle\left\langle\chi_{\max }\right|
$$

where $\mu_{\max }=\max \left\{\mu_{i}\right\}$ is the largest Schmidt coefficient of $|\Psi\rangle$ and $\left|\varphi_{\max }\right\rangle,\left|\chi_{\max }\right\rangle$ are the Schmidt vectors corresponding to $\mu_{\max }$ in Eq. (53). If the largest Schmidt coefficient is degenerate, $|\Psi\rangle$ admits an infinite family of closest classical-quantum states for the Bures distance, formed by convex combinations of the states given in Eq. (59). Remarkably, these states are also the closest separable states to $|\Psi\rangle$. This means that $D_{\mathrm{Bu}}^{G}$ coincides for pure states with the Bures geometric measure of entanglement $E_{\mathrm{Bu}}^{G}(\varrho) \equiv \min _{\sigma_{\mathrm{s}}} d_{\mathrm{Bu}}\left(\varrho, \sigma_{\mathrm{s}}\right)^{2}$ [59], where the minimum is over all separable states $\sigma_{\mathrm{s}}$. It has been shown in Ref. [53 that the latter entanglement measure is simply related to the convex-roof extension $E^{W}(\varrho)$ to mixed states $\varrho$ of the Wei-Goldbart geometric entanglement by $E_{\mathrm{Bu}}^{G}(\varrho)=2-2\left(1-E^{W}(\varrho)\right)^{1 / 2}$. As already remarked in Sec. IIIC, this implies that the Bures geometric discord is a bona fide measure of quantum correlations, just like the Hellinger geometric discord.

We now proceed to establish Theorem 1

Proof. Let us first prove part (b) of the theorem. From Eqs. (4) and (5) it follows that

$$
D_{\mathrm{He}}^{G}(\varrho)=2-2 \max _{\sigma_{\mathrm{cq}} \in \mathcal{C} \mathcal{Q}} \operatorname{Tr} \sqrt{\varrho} \sqrt{\sigma_{\mathrm{cq}}} .
$$

By using the spectral decompositions of the states $\varrho_{B \mid i}$ in Eq. (1), any classical-quantum state can be written as

$$
\sigma_{\mathrm{cq}}=\sum_{i=1}^{n_{A}} \sum_{j=1}^{n_{B}} q_{i j}\left|\alpha_{i}\right\rangle\left\langle\alpha_{i}|\otimes| \beta_{j \mid i}\right\rangle\left\langle\beta_{j \mid i}\right|
$$


where $\left\{q_{i j}\right\}$ is a probability distribution, $\left\{\left|\alpha_{i}\right\rangle\right\}_{i=1}^{n_{A}}$ is an orthonormal basis for $A$ and, for any $i,\left\{\left|\beta_{j \mid i}\right\rangle\right\}_{j=1}^{n_{B}}$ is an orthonormal basis for $B$ (note that the $\left|\beta_{j \mid i}\right\rangle$ need not be orthogonal for distinct $i$ 's). The square root of $\sigma_{\text {cq }}$ is obtained by replacing $q_{i j}$ by $\sqrt{q_{i j}}$ in the r.h.s. of Eq. (61). Hence

$$
\operatorname{Tr} \sqrt{\varrho} \sqrt{\sigma_{\mathrm{cq}}}=\sum_{i, j} \sqrt{q_{i j}}\left\langle\alpha_{i} \otimes \beta_{j \mid i}|\sqrt{\varrho}| \alpha_{i} \otimes \beta_{j \mid i}\right\rangle \leq\left(\sum_{i, j}\left\langle\alpha_{i} \otimes \beta_{j \mid i}|\sqrt{\varrho}| \alpha_{i} \otimes \beta_{j \mid i}\right\rangle^{2}\right)^{\frac{1}{2}} .
$$

The last bound follows from the Cauchy-Schwarz inequality and the identity $\sum_{i, j} q_{i j}=1$. It is saturated when

$$
q_{i j}=\frac{\left\langle\alpha_{i} \otimes \beta_{j \mid i}|\sqrt{\varrho}| \alpha_{i} \otimes \beta_{j \mid i}\right\rangle^{2}}{\sum_{i, j}\left\langle\alpha_{i} \otimes \beta_{j \mid i}|\sqrt{\varrho}| \alpha_{i} \otimes \beta_{j \mid i}\right\rangle^{2}} .
$$

Therefore,

$$
\max _{\left\{q_{i j}\right\}} \operatorname{Tr} \sqrt{\varrho} \sqrt{\sigma_{\mathrm{cq}}}=\left(\sum_{i, j}\left\langle\beta_{j \mid i}\left|B_{i}\right| \beta_{j \mid i}\right\rangle^{2}\right)^{\frac{1}{2}}
$$

with $B_{i}=\left\langle\alpha_{i}|\sqrt{\varrho}| \alpha_{i}\right\rangle$. Note that $B_{i}$ is a self-adjoint operator acting on $\mathcal{H}_{B}$. Now, for any fixed $i$, one has

$$
\sum_{j}\left\langle\beta_{j \mid i}\left|B_{i}\right| \beta_{j \mid i}\right\rangle^{2} \leq \operatorname{Tr}\left[B_{i}^{2}\right] .
$$

This inequality is saturated when $\left\{\left|\beta_{j \mid i}\right\rangle\right\}$ is an eigenbasis of $B_{i}$. Maximizing over all classical-quantum states amounts to maximize over all probability distributions $\left\{q_{i j}\right\}$ and all orthonormal bases $\left\{\left|\alpha_{i}\right\rangle\right\}$ and $\left\{\left|\beta_{j \mid i}\right\rangle\right\}$. Thus

$$
\left(1-\frac{D_{\mathrm{He}}^{G}(\varrho)}{2}\right)^{2}=\max _{\left\{\left|\alpha_{i}\right\rangle\right\}} \sum_{i} \operatorname{Tr}_{B}\left[\left\langle\alpha_{i}|\sqrt{\varrho}| \alpha_{i}\right\rangle^{2}\right] \text {. }
$$

Eq. (56) in Theorem 1 follows immediately from this relation. The closest classical-quantum state $\sigma_{\mathrm{He}, \varrho}$ to $\varrho$ is given by Eq. (61) where $\left|\alpha_{i}\right\rangle=\left|\alpha_{i}^{\text {opt }}\right\rangle$ are the vectors realizing the maximum in Eq. (66), $\left|\beta_{j \mid i}\right\rangle=\left|\beta_{j \mid i}^{\text {opt }}\right\rangle$ are the eigenvectors of $B_{i}^{\mathrm{opt}}=\left\langle\alpha_{i}^{\mathrm{opt}}|\sqrt{\varrho}| \alpha_{i}^{\mathrm{opt}}\right\rangle$, and (see Eq. (63) ):

$$
q_{i j}=\frac{\left\langle\beta_{j \mid i}^{\mathrm{opt}}\left|\left(B_{i}^{\mathrm{opt}}\right)^{2}\right| \beta_{j \mid i}^{\mathrm{opt}}\right\rangle}{\sum_{i} \operatorname{Tr}\left[\left(B_{i}^{\mathrm{opt}}\right)^{2}\right]} .
$$

The expression for $\sigma_{\mathrm{He}, \varrho}$ in Theorem 1 readily follows.

We now establish part (a) of the Theorem. Let $\varrho=|\Psi\rangle\langle\Psi|$ be a pure state with reduced $\operatorname{state}_{\varrho_{A}}=\operatorname{Tr}_{B}|\Psi\rangle\langle\Psi|$. Then $B_{i}=\left|\beta_{i}\right\rangle\left\langle\beta_{i}\right|$, where $\left|\beta_{i}\right\rangle=\left\langle\alpha_{i} \mid \Psi\right\rangle$ has square norm $\left\|\beta_{i}\right\|^{2}=\left\langle\alpha_{i}\left|\varrho_{A}\right| \alpha_{i}\right\rangle$. Thus Eq. (66) yields

$$
\left(1-\frac{D_{\mathrm{He}}^{G}(|\Psi\rangle)}{2}\right)^{2}=\max _{\left\{\left|\alpha_{i}\right\rangle\right\}} \sum_{i}\left\langle\alpha_{i}\left|\varrho_{A}\right| \alpha_{i}\right\rangle^{2} .
$$

In analogy with Eq. (65), the sum in the r.h.s. is bounded from above by $\operatorname{Tr} \varrho_{A}^{2}=K(|\Psi\rangle)^{-1}$, and the bound is saturated when $\left\{\left|\alpha_{i}\right\rangle\right\}$ is an eigenbasis of $\varrho_{A}$. This leads to Eq. (54). The closest classical-quantum state to $|\Psi\rangle$ is given by Eq. (57) with $\left|\alpha_{i}\right\rangle=\left|\varphi_{i}\right\rangle$. In view of the Schmidt decomposition Eq. (53), one obtains Eq. (55).

\section{B. Relation to the Hilbert-Schmidt geometric discord}

The Hilbert-Schmidt geometric discord can be determined in a similar way as the Hellinger geometric discord. Let us give here for completeness a self-contained short derivation of the result, originally derived in Ref. [20].

By definition, the Hilbert-Schmidt geometric discord is

$$
D_{\mathrm{HS}}^{G}(\varrho)=\min _{\sigma_{\mathrm{cq}} \in \mathcal{C} Q}\left\|\varrho-\sigma_{\mathrm{cq}}\right\|_{\mathrm{HS}}^{2}=\operatorname{Tr} \varrho^{2}+\min _{\sigma_{\mathrm{cq}} \in \mathcal{C} Q} \operatorname{Tr}\left(\sigma_{\mathrm{cq}}^{2}-2 \varrho \sigma_{\mathrm{cq}}\right) .
$$

Thanks to Eq. (61), the last trace is equal to

$$
\sum_{i, j}\left\{\left(q_{i j}-\left\langle\alpha_{i} \otimes \beta_{j \mid i}|\varrho| \alpha_{i} \otimes \beta_{j \mid i}\right\rangle\right)^{2}-\left\langle\alpha_{i} \otimes \beta_{j \mid i}|\varrho| \alpha_{i} \otimes \beta_{j \mid i}\right\rangle^{2}\right\} .
$$


The minimum over the probability distribution $\left\{q_{i j}\right\}$ is obviously achieved for $q_{i j}=\left\langle\alpha_{i} \otimes \beta_{j \mid i}|\varrho| \alpha_{i} \otimes \beta_{j \mid i}\right\rangle$. Minimizing also over the orthonormal bases $\left\{\left|\alpha_{i}\right\rangle\right\}$ and $\left\{\left|\beta_{j \mid i}\right\rangle\right\}$ and using Eq. 650 again, one finds

$$
D_{\mathrm{HS}}^{G}(\varrho)=\operatorname{Tr} \varrho^{2}-\max _{\left\{\left|\alpha_{i}\right\rangle\right\}} \sum_{i=1}^{n_{A}} \operatorname{Tr}_{B}\left\langle\alpha_{i}|\varrho| \alpha_{i}\right\rangle^{2}=\min _{\left\{\left|\alpha_{i}\right\rangle\right\}} \sum_{i \neq j}^{n_{A}} \operatorname{Tr}_{B}\left|\left\langle\alpha_{i}|\varrho| \alpha_{j}\right\rangle\right|^{2},
$$

which is the expression originally found by Luo and $\mathrm{Fu}\left[20\right.$. The last equality follows from the relation Tr $\varrho^{2}=$ $\sum_{i, j} \operatorname{Tr}_{B}\left|\left\langle\alpha_{i}|\varrho| \alpha_{j}\right\rangle\right|^{2}$

By the same argument as above, the closest classical-quantum state $\sigma_{\mathrm{HS}, \varrho}$ to $\varrho$ according to the Hilbert-Schmidt distance coincides with the post-measurement state after a local measurement on $A$, namely

$$
\sigma_{\mathrm{HS}, \varrho}=\varrho_{\mathrm{p} . \mathrm{m} .}^{\mathrm{opt}}=\sum_{i=1}^{n_{A}}\left|\alpha_{i}^{\mathrm{opt}}\right\rangle\left\langle\alpha_{i}^{\mathrm{opt}}\right| \otimes\left\langle\alpha_{i}^{\mathrm{opt}}|\varrho| \alpha_{i}^{\mathrm{opt}}\right\rangle,
$$

where the measurement basis $\left\{\left|\alpha_{i}^{\mathrm{opt}}\right\rangle\right\}$ is the orthonormal basis of $A$ maximizing the first sum in Eq. (71). Therefore, as already observed in Ref. [20], the Hilbert-Schmidt geometric discord $D_{\mathrm{HS}}^{G}$ and the measurement-induced geometric discord $D_{\mathrm{HS}}^{M}$ coincide, whatever the space dimensions of the two subsystems.

Next, by comparing Eqs. (71) and (56), one easily deduces the following result:

Theorem 2. For any bipartite state $\varrho$ of a composite system AB, the Hellinger geometric discord $D_{\mathrm{He}}^{G}(\varrho)$ is related to the Hilbert-Schmidt geometric discord $D_{\mathrm{HS}}^{G}(\sqrt{\varrho})$ of the square root of $\varrho$ by

$$
D_{\mathrm{He}}^{G}(\varrho)=g^{-1}\left(2 D_{\mathrm{HS}}^{G}(\sqrt{\varrho})\right) \equiv 2-2\left(1-D_{\mathrm{HS}}^{G}(\sqrt{\varrho})\right)^{\frac{1}{2}}
$$

Note that the Hilbert-Schmidt geometric discord is evaluated for the square root of $\varrho$, which is not a state but is nevertheless a non-negative operator. Thus $\sigma=\sqrt{\varrho} / \operatorname{Tr} \sqrt{\varrho}$ is a density operator and $D_{\mathrm{HS}}^{G}(\sqrt{\varrho})$ is defined as

$$
D_{\mathrm{HS}}^{G}(\sqrt{\varrho}) \equiv\|\sqrt{\varrho}\|_{\mathrm{Tr}}^{2} D_{\mathrm{HS}}^{G}(\sigma)=D_{\mathrm{HS}}^{M}(\sqrt{\varrho})
$$

where $D_{\mathrm{HS}}^{M}(\sqrt{\varrho})$ is given by replacing $\varrho$ by $\sqrt{\varrho}$ in Eq. (6) .

For pure states, Eq. (73) yields a direct relation between the Hellinger and Hilbert-Schmidt geometric discords. Namely,

$$
D_{\mathrm{He}}^{G}(|\Psi\rangle)=2-2\left(1-D_{\mathrm{HS}}^{G}(|\Psi\rangle)\right)^{\frac{1}{2}}
$$

Consequently, as a further corollary, Eq. (154) can be recast in the form $D_{\mathrm{HS}}^{G}(|\Psi\rangle)=1-K(|\Psi\rangle)^{-1}$, a result already known in the literature, see e.g. Refs. [17, 60].

As explained in Sec. IIC the calculation of $D_{\mathrm{He}}^{G}(\varrho)$ is straightforward for qubit-qudit states $\varrho$ once one has determined the decomposition Eq. (18) of the square root of $\varrho$. One can use for this purpose the formula given in Eq. (20). An alternative derivation of this formula for two-qubit states may be obtained by combining Eq. (73) with the result of Ref. [7] on the Hilbert-Schmidt geometric discord. Since a generalization of the latter result to bipartite systems with arbitrary finite space dimensions $n_{A}$ and $n_{B}$ is available [20], a corresponding formula for $D_{\text {He }}^{G}(\varrho)$ for higher-dimensional systems can be obtained as well.

\section{Comparison between the Bures and Hellinger geometric discords}

Theorem 3. Let us recall the increasing function $g(d)$ defined in Eq. (23), and its inverse $g^{-1}(d) \equiv 2-2 \sqrt{1-d / 2}$. The Bures and Hellinger geometric discords satisfy

$$
g^{-1}\left(D_{\mathrm{He}}^{G}(\varrho)\right) \leq D_{\mathrm{Bu}}^{G}(\varrho) \leq D_{\mathrm{He}}^{G}(\varrho)
$$

In particular, $D_{\mathrm{Bu}}^{G}(\varrho)$ lies in the interval bounded by $D_{\mathrm{He}}^{G}(\varrho) / 2$ and $D_{\mathrm{He}}^{G}(\varrho)$. Note that the result of Theorem 3 can be rewritten as:

$$
D_{\mathrm{Bu}}^{G}(\varrho) \leq D_{\mathrm{He}}^{G}(\varrho) \leq g\left(D_{\mathrm{Bu}}^{G}(\varrho)\right) .
$$

Proof. This is a consequence of Eq. (50) and of the Barnum-Knill upper bound on the probability of success in quantum state discrimination [61]. According to such bound, the maximum probability of success $P_{\mathrm{S}}^{\text {opt v.N. }}\left(\left\{\varrho_{i}, \eta_{i}\right\}\right)$ 
is at most equal to the square root of the probability of success obtained by discriminating the states $\varrho_{i}$ with the least-square measurement. This yields (see Ref. [15] for more details):

$$
\max _{\left\{\left|\alpha_{i}\right\rangle\right\}} \sum_{i=1}^{n_{A}} \operatorname{Tr}_{B}\left\langle\alpha_{i}|\sqrt{\varrho}| \alpha_{i}\right\rangle^{2} \leq F(\varrho, \mathcal{C Q}) \leq \max _{\left\{\left|\alpha_{i}\right\rangle\right\}}\left\{\sum_{i=1}^{n_{A}} \operatorname{Tr}_{B}\left\langle\alpha_{i}|\sqrt{\varrho}| \alpha_{i}\right\rangle^{2}\right\}^{\frac{1}{2}} .
$$

The second inequality in Eq. (78) together with Eqs. (49) and (56) lead to the first bound in Eq. (766). The second bound in Eq. (76) is an immediate consequence of the fact that the Bures distance is always smaller or at most equal to the Hellinger distance. We remark for completeness that by exploiting Eqs. (49) and (56), this second bound is equivalent precisely to the lower bound in Eq. (78).

\section{MEASUREMENT-INDUCED GEOMETRIC DISCORD}

In this Section we derive an upper bound on the measurement-induced geometric discord $D^{M}$ in terms of the geometric discord $D^{G}$, both for the Hellinger and the Bures distances. We also determine for these two metrics the value that $D^{M}$ acquires for a pure state $|\Psi\rangle$ and the closest post-measurement state to $|\Psi\rangle$ for local measurements.

\section{A. Hellinger measurement-induced geometric discord}

In view of the definitions in Eqs. (4) and (6), the measurement-induced geometric discord based on the Hellinger distance can be expressed as

$$
D_{\mathrm{He}}^{M}(\varrho)=2-2 \max _{\left\{\left|\alpha_{i}\right\rangle\right\}} \sum_{i=1}^{n_{A}} \operatorname{Tr}_{B}\left[\left\langle\alpha_{i}|\sqrt{\varrho}| \alpha_{i}\right\rangle \sqrt{\left\langle\alpha_{i}|\varrho| \alpha_{i}\right\rangle}\right] .
$$

Here, we have used the expression $\left(\varrho_{\mathrm{p} . \mathrm{m} .}^{\left\{\left|\alpha_{i}\right\rangle\right\}}\right)^{1 / 2}=\sum_{i}\left|\alpha_{i}\right\rangle\left\langle\alpha_{i}\right| \otimes \sqrt{\left\langle\alpha_{i}|\varrho| \alpha_{i}\right\rangle}$ of the square root of the post-measurement state in Eq. (6). Let us first study the restriction of $D_{\mathrm{He}}^{M}$ to pure states.

Theorem 4. On pure states, the Hellinger measurement-induced geometric discord is given by

$$
D_{\mathrm{He}}^{M}(|\Psi\rangle)=2-2 \sum_{i=1}^{n} \mu_{i}^{\frac{3}{2}}
$$

where $\mu_{i}$ are the Schmidt coefficients of $|\Psi\rangle$. The measurement basis $\left\{\left|\alpha_{i}^{\mathrm{opt}}\right\rangle\right\}$ on subsystem $A$ which produces the closest post-measurement state to $|\Psi\rangle$ is the orthonormal basis $\left\{\left|\varphi_{i}\right\rangle\right\}$ formed by the Schmidt vectors in Eq. (53) (i.e., the eigenbasis of the reduced state $\left.\left[\varrho_{\Psi}\right]_{A}\right)$.

As a consequence, the post-measurement state closest to $|\Psi\rangle$ after a local von Neumann measurement on party $A$ takes the form

$$
[\varrho \Psi]_{\mathrm{p} . \mathrm{m} .}^{\mathrm{opt} . \mathrm{He}}=\sum_{i=1}^{n} \mu_{i}\left|\varphi_{i}\right\rangle\left\langle\varphi_{i}|\otimes| \chi_{i}\right\rangle\left\langle\chi_{i}\right|
$$

With the exception of the uniform case $\mu_{i}=1 / n \forall i$, that is, in all cases in which $|\Psi\rangle$ is not maximally entangled, $\left[\varrho_{\Psi}\right]_{\text {p.m. }}^{\text {opt,He }}$ is distinct from the closest classical-quantum state to $|\Psi\rangle$ (compare with Eq. (55)). Therefore, for such non-maximally entangled pure states, $D_{\mathrm{He}}^{M}(|\Psi\rangle)$ is always strictly larger than the Hellinger geometric discord $D_{\mathrm{He}}^{G}(|\Psi\rangle)$.

Proof. Equation (79) yields for $\varrho_{\Psi}=|\Psi\rangle\langle\Psi|$

$$
D_{\mathrm{He}}^{M}(|\Psi\rangle)=2-2 \max _{\left\{\left|\alpha_{i}\right\rangle\right\}} \sum_{i=1}^{n_{A}}\left\|\beta_{i}\right\|^{3},
$$

with the unnormalized vector $\left|\beta_{i}\right\rangle$ in the Hilbert space of $B$ defined as $\left|\beta_{i}\right\rangle=\left\langle\alpha_{i} \mid \Psi\right\rangle$. The Schmidt decomposition gives

$$
\left\|\beta_{i}\right\|^{2}=\sum_{j=1}^{n} \mu_{j}\left|\left\langle\alpha_{i} \mid \varphi_{j}\right\rangle\right|^{2} \leq\left(\sum_{j=1}^{n} \mu_{j}^{\frac{3}{2}}\left|\left\langle\alpha_{i} \mid \varphi_{j}\right\rangle\right|^{2}\right)^{\frac{2}{3}}
$$


where the upper bound is obtained by combining the Hölder inequality and $\sum_{j}\left|\left\langle\alpha_{i} \mid \varphi_{j}\right\rangle\right|^{2} \leq 1$. It follows that

$$
\sum_{i=1}^{n_{A}}\left\|\beta_{i}\right\|^{3} \leq \sum_{j=1}^{n} \mu_{j}^{\frac{3}{2}}
$$

This bound is saturated by taking $\left|\alpha_{i}\right\rangle=\left|\varphi_{i}\right\rangle$ for all $i=1, \ldots, n$, with $n=\min \left\{n_{A}, n_{B}\right\}$ (if $n<n_{A}$, the remaining vectors $\left|\alpha_{i}\right\rangle$ are chosen arbitrarily to form an orthonormal basis of $\mathcal{H}_{A}$ ). Equation (80) then follows upon replacing the sum in Eq. (82) by its upper bound.

Although we already know from Theorem 2.4 of Ref. [16] that $D_{\mathrm{He}}^{M}$ satisfies Axiom (iv) (see Sec. [IIC), it is instructive to deduce this result from Eq. (80). Introducing the unitarily invariant function $f\left(\varrho_{A}\right)=2-2 \operatorname{Tr} \varrho_{A}^{3 / 2}$ on the set of density operators on $\mathcal{H}_{A}$, this equation can be rewritten as $D_{\mathrm{He}}^{M}(|\Psi\rangle)=f\left(\left[\varrho_{\Psi}\right]_{A}\right)$. The fact that this quantity is an entanglement monotone follows directly from the characterization of convex strongly monotone entanglement measures provided by Vidal [54]. Indeed, according to Ref. [54], $E(|\Psi\rangle)=f\left(\left[\varrho_{\Psi}\right]_{A}\right)$ defines an entanglement monotone on pure states if $\varrho_{A} \mapsto f\left(\varrho_{A}\right)$ is concave (notice, however, that this condition is not necessary and sufficient: notable exceptions are provided by the logarithmic negativity, which is not convex but is nevertheless strongly entanglement monotone, see Ref. 62], and the Bures geometric measure of entanglement $E_{\mathrm{Bu}}^{G}$, which is convex but entanglement monotone in the weak sense discussed in Section [IIC, see Ref. [15]). The concavity of $\varrho_{A} \mapsto f\left(\varrho_{A}\right)$ is a consequence of the convexity of $\varrho_{A} \mapsto \operatorname{Tr} k\left(\varrho_{A}\right)$ for real convex functions $k$, in particular for $k(x)=x^{3 / 2}$, as proved for instance in Ref. [63]. Hence $D_{\mathrm{He}}^{M}$ satisfies Axiom (iv) of Section I. Since the Hellinger distance is contractive under CPTP maps, $D_{\mathrm{He}}^{M}$ also fulfills Axioms (i-iii) (see Sec. IIIC). Summing up, $D_{\mathrm{He}}^{M}$ is a bona fide measure of quantum correlations. The maximum value of $D_{\mathrm{He}}^{M}$ when $n_{A} \leq n_{B}$ is equal to $2-2 / \sqrt{n_{A}}$, as reported in Table (this follows from Eq. (80) and the fact that $D_{\mathrm{He}}^{M}$ is maximum for maximally entangled pure states).

Theorem 5. The Hellinger geometric discord and Hellinger measurement-induced geometric discord satisfy

$$
D_{\mathrm{He}}^{G}(\varrho) \leq D_{\mathrm{He}}^{M}(\varrho) \leq g\left(D_{\mathrm{He}}^{G}(\varrho)\right),
$$

with the function $g(d)$ defined by Eq. (23).

In particular, the ratio $D_{\mathrm{He}}^{M}(\varrho) / D_{\mathrm{He}}^{G}(\varrho)$ always lies in the interval $[1,2]$.

Proof. The first inequality follows trivially from the definitions. By the operator concavity of $f(x)=\sqrt{x}$ and the Jensen-type inequality applied to the CPTP map $\varrho \mapsto \sum_{i}\left|\alpha_{i}\right\rangle\left\langle\alpha_{i}|\otimes \mathbb{1} \varrho| \alpha_{i}\right\rangle\left\langle\alpha_{i}\right| \otimes \mathbb{1}$ (see, e.g., Refs. [15, 63]), the following operator bound holds

$$
\sqrt{\left\langle\alpha_{i}|\varrho| \alpha_{i}\right\rangle} \geq\left\langle\alpha_{i}|\sqrt{\varrho}| \alpha_{i}\right\rangle .
$$

As a consequence, the trace in Eq. (79) is bounded from below by $\operatorname{Tr}\left\langle\alpha_{i}|\sqrt{\varrho}| \alpha_{i}\right\rangle^{2}$. Comparing with the expression Eq. (56) of the Hellinger geometric discord, the upper bound on $D_{\mathrm{He}}^{M}$ follows.

\section{B. Bures measurement-induced geometric discord}

For any state $\varrho$ of the bipartite system $A B$, let us denote by $\mathcal{L} \mathcal{M}_{\varrho}$ the set of all post-measurement states obtained from $\varrho$ after local rank-one projective measurements on $A$, that is, the states given by Eq. (6). In analogy with Eq. (49), the Bures measurement-induced geometric discord is equal to $D_{\mathrm{Bu}}^{M}(\varrho)=2-2 \sqrt{F\left(\varrho, \mathcal{L} \mathcal{M}_{\varrho}\right)}$, where $F\left(\varrho, \mathcal{L} \mathcal{M}_{\varrho}\right)$ is the maximum fidelity between $\varrho$ and a state belonging to $\mathcal{L} \mathcal{M}_{\varrho}$. One easily finds

$$
F\left(\varrho, \mathcal{L} \mathcal{M}_{\varrho}\right)=\max _{\left\{\left|\alpha_{i}\right\rangle\right\}}\left\{\operatorname{Tr} \sqrt{\sum_{i=1}^{n_{A}} \eta_{i}^{2} \varrho_{i}^{2}}\right\}^{2},
$$

with the states $\varrho_{i}$ and probabilities $\eta_{i}$ given by Eq. (11). This proves Eq. (13) reported in Section the following theorem holds:

Theorem 6. (a) On pure states, the Bures measurement-induced geometric discord is given by

$$
D_{\mathrm{Bu}}^{M}(|\Psi\rangle)=D_{\mathrm{He}}^{G}(|\Psi\rangle)=2-2 K(|\Psi\rangle)^{-\frac{1}{2}},
$$

where $K(|\Psi\rangle)=\left(\sum_{i} \mu_{i}^{2}\right)^{-1}$ is the Schmidt number of $|\Psi\rangle$. In particular, $D_{\mathrm{Bu}}^{M}$ satisfies Axiom (iv) of Section $\square$ and is thus a bona fide measure of quantum correlations. Moreover, the measurement basis $\left\{\left|\alpha_{i}^{\text {opt }}\right\rangle\right\}$ which produces the closest post-measurement state $\left[\varrho_{\Psi}\right]_{\mathrm{p} . \mathrm{m} .}^{\mathrm{opt}} \in \mathcal{L} \mathcal{L M}_{\Psi}$ to $|\Psi\rangle$ for the Bures distance is the eigenbasis $\left\{\left|\varphi_{i}\right\rangle\right\}$ of the reduced state $\left[\varrho_{\Psi}\right]_{A}$. 
(b) For any mixed state $\varrho$, if $n_{A} \leq n_{B}$ then the maximum value $D_{\max }$ of $D_{\mathrm{Bu}}^{M}(\varrho)$ is $D_{\max }=2-2 / \sqrt{n_{A}}$. Moreover, $D_{\mathrm{Bu}}^{M}(\varrho)=D_{\max }$ if and only if $\varrho$ has maximum entanglement of formation $E_{\mathrm{EoF}}(\varrho)=\ln n_{A}$. Thus, $D_{\mathrm{Bu}}^{M}$ is a proper measure of quantum correlations that, besides Axioms (i)-(iv), satisfies also the additional Axiom (v).

Quite remarkably, the post-measurement state $\left[\varrho_{\Psi}\right]_{\mathrm{p}, \mathrm{m}}^{\mathrm{opt}} \in \mathcal{L} \mathcal{M}_{\varrho}$ which is closest to the pure state $|\Psi\rangle$ is the same for the Hellinger, Bures, trace, and Hilbert-Schmidt distances. The explicit expression of this state is given by Eq. (81). This is a consequence of Theorem 4, Theorem 6, and Theorem 3.3. of Ref. [16] for the three first distances. For the Hilbert-Schmidt distance, this follows from Eqs. (71) and (72) and from the bound in Eq. (90) below.

Comparing Eqs. (58) and (88) we find that, as for the case of the Hellinger distance, the trivial bound $D_{\mathrm{Bu}}^{G}(|\Psi\rangle) \leq$ $D_{\mathrm{Bu}}^{M}(|\Psi\rangle)$ is strict for all non-maximally entangled pure states.

Let us also stress that statement (b) holds as well for the Bures geometric discord $D_{\mathrm{Bu}}^{G}$, which has the same maximum value $D_{\max }$ when $n_{A} \leq n_{B}$ (see Sec. [IID).

Proof. We first consider the case of pure states: setting $\varrho=|\Psi\rangle\langle\Psi|$ in Eq. (87), we have

$$
F\left(|\Psi\rangle, \mathcal{L} \mathcal{M}_{\Psi}\right)=\max _{\left\{\left|\alpha_{i}\right\rangle\right\}} \sum_{i=1}^{n_{A}}\left\|\beta_{i}\right\|^{4}
$$

with $\left|\beta_{i}\right\rangle=\left\langle\alpha_{i} \mid \Psi\right\rangle$ as before. Thanks to the first identity in Eq. (83) and to the Cauchy-Schwarz inequality, we also have

$$
\begin{aligned}
\sum_{i}\left\|\beta_{i}\right\|^{4}=\sum_{i, j, k} \mu_{j} \mu_{k}\left|\left\langle\alpha_{i} \mid \varphi_{j}\right\rangle\right|^{2}\left|\left\langle\alpha_{i} \mid \varphi_{k}\right\rangle\right|^{2} & \leq\left(\sum_{i, j, k} \mu_{j}^{2}\left|\left\langle\alpha_{i} \mid \varphi_{j}\right\rangle\right|^{2}\left|\left\langle\alpha_{i} \mid \varphi_{k}\right\rangle\right|^{2}\right)^{\frac{1}{2}}\left(\sum_{i, j, k} \mu_{k}^{2}\left|\left\langle\alpha_{i} \mid \varphi_{j}\right\rangle\right|^{2}\left|\left\langle\alpha_{i} \mid \varphi_{k}\right\rangle\right|^{2}\right)^{\frac{1}{2}} \\
& \leq \sum_{j=1}^{n} \mu_{j}^{2}=K(|\Psi\rangle)^{-1}
\end{aligned}
$$

The bound is saturated by taking $\left|\alpha_{i}\right\rangle=\left|\varphi_{i}\right\rangle$ for $i=1, \ldots, n$, hence the maximum of the l.h.s. coincides with $K(|\Psi\rangle)^{-1}$. The statement that $D_{\mathrm{Bu}}^{M}$ is a bona fide measure of quantum correlations follows from the results of Section $\amalg$ III and the fact that $K(|\Psi\rangle)$ is an entanglement monotone.

We now consider the case of mixed states. The statement (b) follows from Eq. (87) and the following trace inequality: for any finite family of operators $\left\{X_{i}\right\}_{i=1}^{n_{A}}$,

$$
\left\|\sum_{i=1}^{n_{A}} X_{i}\right\|_{\operatorname{Tr}} \leq \sqrt{n_{A}} \operatorname{Tr} \sqrt{\sum_{i=1}^{n_{A}}\left|X_{i}\right|^{2}}
$$

with equality if and only if all $X_{i}$ are equal. This inequality is a consequence of the operator monotonicity of the square root function (see e.g. Refs. [63, 64]) and of the operator bound $\left|\sum_{i=1}^{n_{A}} X_{i}\right|^{2} \leq n_{A} \sum_{i=1}^{n_{A}}\left|X_{i}\right|^{2}$, which in turn follows from $X_{i}^{\dagger} X_{j}+X_{j}^{\dagger} X_{i} \leq\left|X_{i}\right|^{2}+\left|X_{j}\right|^{2}$. Taking $X_{i}=\eta_{i} \varrho_{i}$ in Eq. (91), using Eq. (87), and recalling that $\sum_{i} \eta_{i} \varrho_{i}=\varrho$, one finds that $F\left(\varrho, \mathcal{L} \mathcal{M}_{\varrho}\right) \geq 1 / n_{A}$. Hence $D_{\mathrm{Bu}}^{M}(\varrho) \leq D_{\max }$. Together with the trivial bound $D_{\mathrm{Bu}}^{G}(\varrho) \leq D_{\mathrm{Bu}}^{M}(\varrho)$ and the identity $D_{\mathrm{Bu}}^{G}(\varrho)=D_{\max }$ for maximally entangled states (as $D_{\mathrm{Bu}}^{G}$ satisfies Axiom $(\mathrm{v})$ ), this implies that $D_{\mathrm{Bu}}^{M}(\varrho)=D_{\max }$ on such states. Conversely, let us consider a state $\varrho$ such that $D_{\mathrm{Bu}}^{M}(\varrho)=D_{\max }$, i.e., $F\left(\varrho, \mathcal{L} \mathcal{M}_{\varrho}\right)=1 / n_{A}$. Then the inequality in Eq. (91) with $X_{i}=\eta_{i} \varrho_{i}$ is saturated, so that $\eta_{i} \varrho_{i}$ is independent of $i$, for any orthonormal basis $\left\{\left|\alpha_{i}\right\rangle\right\}$. One deduces from the relations $\operatorname{Tr} \varrho_{i}=1=\sum_{i} \eta_{i}$ and $\sum_{i} \eta_{i} \varrho_{i}=\varrho$ that $\eta_{i}=1 / n_{A}$ and $\varrho_{i}=\varrho$ for any $i=1, \ldots, n_{A}$ and any basis $\left\{\left|\alpha_{i}\right\rangle\right\}$. By using the same arguments as in the proof of the Proposition following Theorem 3 in Ref. [9], one concludes that $\varrho$ is a maximally entangled state according to the entanglement of formation. More specifically, $\varrho$ is a convex combination of maximally entangled pure states $\left|\Psi_{k}\right\rangle$, whose expression is provided by Eq. (114) below and which satisfy the orthogonality conditions given after this equation. Note that such maximally entangled states are not necessarily pure if $n_{B} \geq 2 n_{A}$.

Theorem 7. The Bures geometric discord $D_{\mathrm{Bu}}^{G}$ and the Bures measurement-induced geometric discord $D_{\mathrm{Bu}}^{M}$ satisfy a bound analogous to that established in Theorem 5 for $D_{\mathrm{He}}^{G}$ and $D_{\mathrm{He}}^{M}$, namely:

$$
D_{\mathrm{Bu}}^{G}(\varrho) \leq D_{\mathrm{Bu}}^{M}(\varrho) \leq g\left(D_{\mathrm{Bu}}^{G}(\varrho)\right)
$$

with $g(d)=2 d-d^{2} / 2$.

Let us observe that the lower and upper bounds on $D_{\mathrm{Bu}}^{M}$ in Eq. (92) are identical to the bounds on $D_{\mathrm{He}}^{G}$ in Eq. (77). It is clear that the upper bound is not optimal for strongly quantum-correlated states (in fact, one has $\left.g\left(2-2 / \sqrt{n_{A}}\right)=2-2 / n_{A}>D_{\max }\right)$. On the other hand, this upper bound is optimal in the limit of almost nondiscordant states. Indeed, consider a pure state $\left|\Psi_{\varepsilon}\right\rangle$ with maximum Schmidt coefficient $\mu_{\max }=1-\varepsilon$ with $0 \leq \varepsilon \ll 1$. 
From Eqs. (54), (58), and (88) it follows that $g\left(D_{\mathrm{Bu}}^{G}\left(\left|\Psi_{\varepsilon}\right\rangle\right)\right)=D_{\mathrm{Bu}}^{M}\left(\left|\Psi_{\varepsilon}\right\rangle\right)=D_{\mathrm{He}}^{G}\left(\left|\Psi_{\varepsilon}\right\rangle\right)=2 \varepsilon$ up to terms of order $\varepsilon^{2}$. This means that the upper bounds in Eqs. (77) and (92) are saturated asymptotically by pure states that are arbitrarily close to product states. According to Eq. (16), the r.h.s. of Eq. (92) places an upper bound on $D_{\text {Tr }}^{G}(\varrho) / 2$ as well. However, in this case it is not clear whether the bound can be saturated asymptotically.

If subsystem $A$ is a qubit, inequalities stronger than the upper bounds in Eqs. (777) and (92) can be obtained from Theorems 9 and 11 below, by inserting the expressions (95) and (102) into Eqs. (96) and (104). This yields the improved tight bounds reported in Section IID, given in Eqs. (25) and (28).

Proof. The first inequality is trivial. When the density matrix $\varrho$ is invertible, the second inequality follows by combining Eqs. (50) and (87) with the upper bound by Ogawa and Nagaoka on the maximum probability of success in quantum state discrimination [65]:

$$
P_{\mathrm{S}}^{\mathrm{opt}}\left(\left\{\varrho_{i}, \eta_{i}\right\}\right) \leq \operatorname{Tr} \sqrt{\sum_{i=1}^{n_{A}} \eta_{i}^{2} \varrho_{i}^{2}} .
$$

When $\varrho$ is not invertible, we may approximate it by the invertible density matrix $\varrho_{\varepsilon}=(1-\varepsilon) \varrho+\varepsilon \mathbb{1} /\left(n_{A} n_{B}\right)$ with $\varepsilon \in(0,1]$ and obtain the desired result by continuity, letting $\varepsilon \rightarrow 0$. It is worth noting that it is also known that the maximum probability of success is bounded from below by the square of the r.h.s. of Eq. (93), see Ref. [66]. However, in our context such bound is not very useful, as it yields the trivial inequality $D_{\mathrm{Bu}}^{G}(\varrho) \leq D_{\mathrm{Bu}}^{M}(\varrho)$.

\section{DISCORD OF RESPONSE: COMPUTABLE AND BONA FIDE MEASURE OF QUANTUM CORRELATIONS}

In this Section we show that whenever the reference party $A$ is a qubit or a qutrit, the Hellinger discord of response is a simple function of the Hellinger geometric discord, and the same holds true in the Bures case when $A$ is a qubit (Theorems 8 and 10). As a consequence, the Hellinger discord of response is computable for all qubit-qudit states, as anticipated in Sec. II C

We also derive lower and upper bounds on $D_{\mathrm{He}}^{R}$ valid for arbitrary subsystems $A$ and $B$, first in terms of $D_{\mathrm{He}}^{G}$ (Theorem 8) and then in terms of $D_{\mathrm{Bu}}^{R}$ (Theorem 9). Moreover, we obtain an upper bound on the Bures measurementinduced geometric discord in terms of the Bures discord of response (Theorem 11). Finally, we prove that for the trace distance, all geometric measures $D_{\operatorname{Tr}}^{G}, D_{\operatorname{Tr}}^{M}$, and $D_{\operatorname{Tr}}^{R}$ coincide whenever $A$ is a qubit, and we show that the HilbertSchmidt discord of response $D_{\mathrm{HS}}^{R}$ is always smaller or equal to the trace discord of response $D_{\operatorname{Tr}}^{R}$ (Theorem 12) .

\section{A. Hellinger discord of response: bona fide and computable measure of quantum correlations}

The following theorem yields that the Hellinger discord of response enjoys a simple, exact relation to the Hellinger geometric discord whenever $A$ is a qubit or a qutrit. For subsystems $A$ with space dimensions $n_{A}>3$, there is no direct relation between these two measures (see Appendix $\mathrm{A}$ ), however we are able to derive lower and upper bounds on $D_{\mathrm{He}}^{R}$ in terms of $D_{\mathrm{He}}^{G}$.

Theorem 8. The Hellinger discord of response is bounded in terms of the Hellinger geometric discord as follows:

$$
\sin ^{2}\left(\frac{\pi}{n_{A}}\right) g\left(D_{\mathrm{He}}^{G}(\varrho)\right) \leq D_{\mathrm{He}}^{R}(\varrho) \leq g\left(D_{\mathrm{He}}^{G}(\varrho)\right)
$$

with $g(d)=2 d-d^{2} / 2$. If subsystem $A$ is a qubit or a qutrit then the first inequality is an equality, that is,

$$
D_{\mathrm{He}}^{R}(\varrho)= \begin{cases}g\left(D_{\mathrm{He}}^{G}(\varrho)\right) & \text { if } n_{A}=2 \\ \frac{3}{4} g\left(D_{\mathrm{He}}^{G}(\varrho)\right) & \text { if } n_{A}=3\end{cases}
$$

The proof of this theorem is reported in Appendix A

By combining Eqs. (85) and (94) one obtains the upper bound $D_{\mathrm{He}}^{M}(\varrho) \leq D_{\mathrm{He}}^{R}(\varrho) / \sin ^{2}\left(\pi / n_{A}\right)$ on the Hellinger measurement-induced geometric discord, as reported in Table III. Let us also recall that the closed analytical expression of $D_{\mathrm{He}}^{R}(\varrho)$ for arbitrary qubit-qudit states $\varrho$ in Eq. (22) can be obtained from Eq. (95) and the expression of the Hellinger geometric discord given by Eq. (20).

Interestingly, the Hellinger discord of response provides lower and upper bounds on the Bures discord of response, which is harder to compute. Optimal bounds are provided by the following theorem: 
Theorem 9. For subsystems $A$ and $B$ with arbitrary space dimensions, one has

$$
1-\sqrt{1-D_{\mathrm{He}}^{R}(\varrho)} \leq D_{\mathrm{Bu}}^{R}(\varrho) \leq D_{\mathrm{He}}^{R}(\varrho) .
$$

The first bound is saturated for pure states.

The numerical results reported in Fig. 1(c) indicate that the second bound is almost tight for two-qubit systems.

Proof. The second inequality in the theorem is a trivial consequence of the fact that the Bures distance is bounded from above by the Hellinger distance, see Eq. (39). In order to prove the first inequality, we exploit the definitions of $D_{\mathrm{Bu}}^{R}$ and $D_{\mathrm{He}}^{R}$ (see Eqs. (3) , (44), and (7)) and the identities $\left(U \varrho U^{\dagger}\right)^{\frac{1}{2}}=U \sqrt{\varrho} U^{\dagger}$ and $F\left(\varrho, U \varrho U^{\dagger}\right)=\|\sqrt{\varrho} U \sqrt{\varrho}\|_{\mathrm{Tr}}^{2}$ holding for any unitary operator $U$. This gives

$$
\begin{aligned}
& D_{\mathrm{Bu}}^{R}(\varrho)=1-\max _{U_{A} \in \mathcal{U}_{\Lambda}}\left\|\sqrt{\varrho} U_{A} \otimes \mathbb{1} \sqrt{\varrho}\right\|_{\mathrm{Tr}}, \\
& D_{\mathrm{He}}^{R}(\varrho)=1-\max _{U_{A} \in \mathcal{U}_{\Lambda}} \operatorname{Tr}\left(\sqrt{\varrho} U_{A} \otimes \mathbb{1} \sqrt{\varrho} U_{A}^{\dagger} \otimes \mathbb{1}\right) .
\end{aligned}
$$

We now take advantage of the bound

$$
\left\|\sqrt{\varrho} U_{A} \otimes \mathbb{1} \sqrt{\varrho}\right\|_{\operatorname{Tr}}^{2} \leq \operatorname{Tr}\left(\sqrt{\varrho} U_{A} \otimes \mathbb{1} \sqrt{\varrho} U_{A}^{\dagger} \otimes \mathbb{1}\right)
$$

This bound follows from the identity $\|A\|_{\operatorname{Tr}}=\operatorname{Tr}\left(V^{\dagger} A\right)$ with $V$ a unitary operator such that $A=V|A|=\sqrt{\varrho} U_{A} \otimes \mathbb{1} \sqrt{\varrho}$ (polar decomposition), from the Cauchy-Schwarz inequality $\left|\operatorname{Tr}\left(B^{\dagger} C\right)\right|^{2} \leq \operatorname{Tr}|B|^{2} \operatorname{Tr}|C|^{2}$ with $B=\varrho^{\frac{1}{4}} V \varrho^{\frac{1}{4}}$ and $C=\varrho^{\frac{1}{4}} U_{A} \otimes \mathbb{1} \varrho^{\frac{1}{4}}$, and from $\operatorname{Tr}|B|^{2}=\operatorname{Tr} \sqrt{\varrho} V^{\dagger} \sqrt{\varrho} V \leq \operatorname{Tr} \varrho=1$ (again by the Cauchy-Schwarz inequality). Then, combining Eqs. (97), (98), and (99), it holds that $1-D_{\mathrm{He}}^{R}(\varrho) \geq\left(1-D_{\mathrm{Bu}}^{R}(\varrho)\right)^{2}$. This inequality is an equality for pure states, as can easily be inferred from Eqs. (42) and (46).

\section{B. Bures discord of response}

If $U_{A}$ is a local unitary operator acting on $\mathcal{H}_{A}$ with spectrum $\Lambda$ given by the roots of the unity, then

$$
U_{A}=\sum_{j=1}^{n_{A}} e^{-\mathrm{i} \frac{2 \pi j}{n_{A}}}\left|\alpha_{j}\right\rangle\left\langle\alpha_{j}\right|
$$

for some orthonormal basis $\left\{\left|\alpha_{j}\right\rangle\right\}$ of $\mathcal{H}_{A}$. By inserting this spectral decomposition into Eq. (97) we obtain

$$
D_{\mathrm{Bu}}^{R}(\varrho)=1-\max _{\left\{\left|\alpha_{i}\right\rangle\right\}}\left\|\sum_{j=1}^{n_{A}} \eta_{j} e^{-\mathrm{i} \frac{2 \pi j}{n_{A}}} \varrho_{j}\right\|_{\mathrm{Tr}}
$$

with the states $\varrho_{i}$ and probabilities $\eta_{i}$ given by Eq. (11). This proves the general expression of $D_{\mathrm{Bu}}^{R}(\varrho)$ anticipated in Section IIB.

Theorem 10. If $A$ is a qubit $\left(n_{A}=2\right)$ and $B$ is a qudit $\left(n_{B} \geq 2\right)$, then the Bures discord of response is related to the Bures geometric discord by

$$
D_{\mathrm{Bu}}^{R}(\varrho)=g\left(D_{\mathrm{Bu}}^{G}(\varrho)\right)
$$

with $g(d)=2 d-d^{2} / 2$.

Proof. The proof relies on Eq. (50) and the Helstrom formula Eq. (52). Accordingly, the maximum fidelity $F(\varrho, \mathcal{C Q})=$ $\left(1-D_{\mathrm{Bu}}^{G}(\varrho) / 2\right)^{2}$ between $\varrho$ and a classical-quantum state is given for $n_{A}=2$ by

$$
F(\varrho, \mathcal{C Q})=\frac{1}{2}\left(1+\max _{\left\{\left|\alpha_{i}\right\rangle\right\}}\left\|\eta_{2} \varrho_{2}-\eta_{1} \varrho_{1}\right\|_{\operatorname{Tr}}\right) .
$$

For $n_{A}=2$, the two operators inside the trace norms in Eqs. (101) and (103) coincide and one obtains Eq. (102).

An optimal upper bound on the measurement-induced geometric discord $D_{\mathrm{Bu}}^{M}$ in terms of $D_{\mathrm{Bu}}^{R}$ is given by: 
Theorem 11. If $A$ is a qubit or a qutrit $\left(n_{A}=2\right.$ or $\left.n_{A}=3\right)$ and $B$ is a qudit $\left(n_{B} \geq 2\right)$, the Bures measurementinduced geometric discord and the Bures discord of response satisfy

$$
D_{\mathrm{Bu}}^{M}(\varrho) \leq 2-\frac{2}{\sqrt{n_{A}}} \sqrt{1+\left(n_{A}-1\right)\left(1-D_{\mathrm{Bu}}^{R}(\varrho)\right)^{2}} .
$$

The bound is saturated for pure states. For $n_{A}>3$, the following weaker bound holds:

$$
D_{\mathrm{Bu}}^{M}(\varrho) \leq 2-\frac{2}{\sqrt{n_{A}}}\left(1-D_{\mathrm{Bu}}^{R}(\varrho)\right) \text {. }
$$

Proof. The second bound is valid for any space dimension $n_{A}$. It follows from the expressions for $D_{\mathrm{Bu}}^{M}(\varrho)$ and $D_{\mathrm{Bu}}^{R}(\varrho)$ given in Eqs. (87) and (101), and from the inequality in Eq. (91). We now show that when $n_{A}=2$ or 3 , the stronger bound of Eq. (104) holds. In view of Eqs. (87) and (101), it is enough to show that

$$
n_{A}\left\|\sqrt{\sum_{j=1}^{n_{A}} \eta_{j}^{2} \varrho_{j}^{2}}\right\|_{\mathrm{Tr}}^{2} \geq 1+\left(n_{A}-1\right)\left\|\sum_{j=1}^{n_{A}} e^{-\mathrm{i} \frac{2 \pi j}{n_{A}}} \eta_{j} \varrho_{j}\right\|_{\mathrm{Tr}}^{2} .
$$

Let us consider the operators

$$
A_{k}=\sum_{j=1}^{n_{A}} e^{-\mathrm{i} \frac{2 \pi j k}{n_{A}}} \eta_{j} \varrho_{j} \quad, \quad k=1, \ldots, n_{A}
$$

Then $\sum_{k=1}^{n_{A}}\left|A_{k}\right|^{2}=n_{A} \sum_{j=1}^{n_{A}} \eta_{j}^{2} \varrho_{j}^{2}$. We now make use of the inverse triangle inequality $\left(\operatorname{Tr} \sqrt{|A|^{2}+|B|^{2}}\right)^{2} \geq$ $(\operatorname{Tr}|A|)^{2}+(\operatorname{Tr}|B|)^{2}$ (see e.g. Ref. 67], Lemma 1), which follows from a majorization argument (see Ref. [64], Exercise II.1.14 and Theorem II.3.1), the concavity of the square root function, and Minkowski's inequality for sequences. Thanks to the inverse triangle inequality, we obtain

$$
n_{A}\left\|\sqrt{\sum_{j=1}^{n_{A}} \eta_{j}^{2} \varrho_{j}^{2}}\right\|_{\mathrm{Tr}}^{2}=\left\|\sqrt{\sum_{k=1}^{n_{A}}\left|A_{k}\right|^{2}}\right\|_{\mathrm{Tr}}^{2} \geq \sum_{k=1}^{n_{A}}\left\|A_{k}\right\|_{\mathrm{Tr}}^{2}=1+\sum_{k=1}^{n_{A}-1}\left\|A_{k}\right\|_{\mathrm{Tr}}^{2},
$$

where we have used $A_{n_{A}}=\sum_{j} \eta_{j} \varrho_{j}=\varrho$ in the last equality. For $n_{A}=2$, the bound in Eq. (106) follows immediately from Eqs. (107) and (108). For $n_{A}=3$, we exploit the identity $A_{2}=A_{1}^{\dagger}$ and the property $\left\|A^{\dagger}\right\|_{\operatorname{Tr}}=\|A\|_{\operatorname{Tr}}$ of the trace norm.

It remains to show that the bound is saturated for pure states $\varrho_{\Psi}=|\Psi\rangle\langle\Psi|$. In view of Eqs. (42) and (43), the Schmidt decomposition of $|\Psi\rangle$ (see Eq. (53)), and the spectral decomposition of $U_{A}$ (see Eq. (100)), we obtain

$$
1+\left(n_{A}-1\right)\left(1-D_{\mathrm{Bu}}^{R}(|\Psi\rangle)\right)^{2}=\max _{\left\{\left|\alpha_{i}\right\rangle\right\}} \sum_{i, j, k, l} \mu_{i} \mu_{k}\left(1+\left(n_{A}-1\right) \cos \left(\frac{2 \pi(j-l)}{n_{A}}\right)\right)\left|\left\langle\varphi_{i} \mid \alpha_{j}\right\rangle\right|^{2}\left|\left\langle\varphi_{k} \mid \alpha_{l}\right\rangle\right|^{2}
$$

If $n_{A}=2$ or 3, only the terms $j=l$ contribute to the sum. Thanks to Eqs. (88) and (90), the r.h.s. of Eq. (109) is equal to $n_{A} K(|\Psi\rangle)^{-1}=n_{A}\left(1-D_{\mathrm{Bu}}^{M}(|\Psi\rangle) / 2\right)^{2}$. Hence the inequality in Eq. (104) is saturated for pure states.

\section{Trace discord of response}

Theorem 12. If party $A$ is a qubit $\left(n_{A}=2\right)$ and party $B$ is a qudit $\left(n_{B} \geq 2\right)$, the trace discord of response, trace geometric discord, and trace measurement-induced geometric discord all coincide:

$$
D_{\operatorname{Tr}}^{R}(\varrho)=D_{\operatorname{Tr}}^{M}(\varrho)=D_{\operatorname{Tr}}^{G}(\varrho)
$$

Furthermore, one has

$$
D_{\operatorname{Tr}}^{R}(\varrho) \geq D_{\mathrm{HS}}^{R}(\varrho) .
$$

It is worth remarking that the bound in Eq. (111) is stronger than the trivial bound $2 D_{\operatorname{Tr}}^{R}(\varrho) \geq D_{\mathrm{HS}}^{R}(\varrho)$ that follows from Eq. (37). Moreover, it is saturated for pure states (see Sect. IID).

The proof of Theorem 12 is reported in Appendix A. It makes explicit use of the fact that the spectrum of the unitary operators in the definition of the discord of response is given by the roots of the unity (harmonic spectrum). The identity $D_{\operatorname{Tr}}^{M}=D_{\operatorname{Tr}}^{G}$ and the fact that it holds only for $n_{A}=2$ have been originally established in Ref. [21].

The trace geometric discord has been computed in different works for specific classes of two-qubit states: a closed expression for Bell-diagonal states has been found in Refs. [21, 56], and it was further generalized to the class of the so-called $X$ states and to the quantum-classical states in Ref. [14]. Due to Theorem 12, we can immediately extend these results to the trace discord of response. 

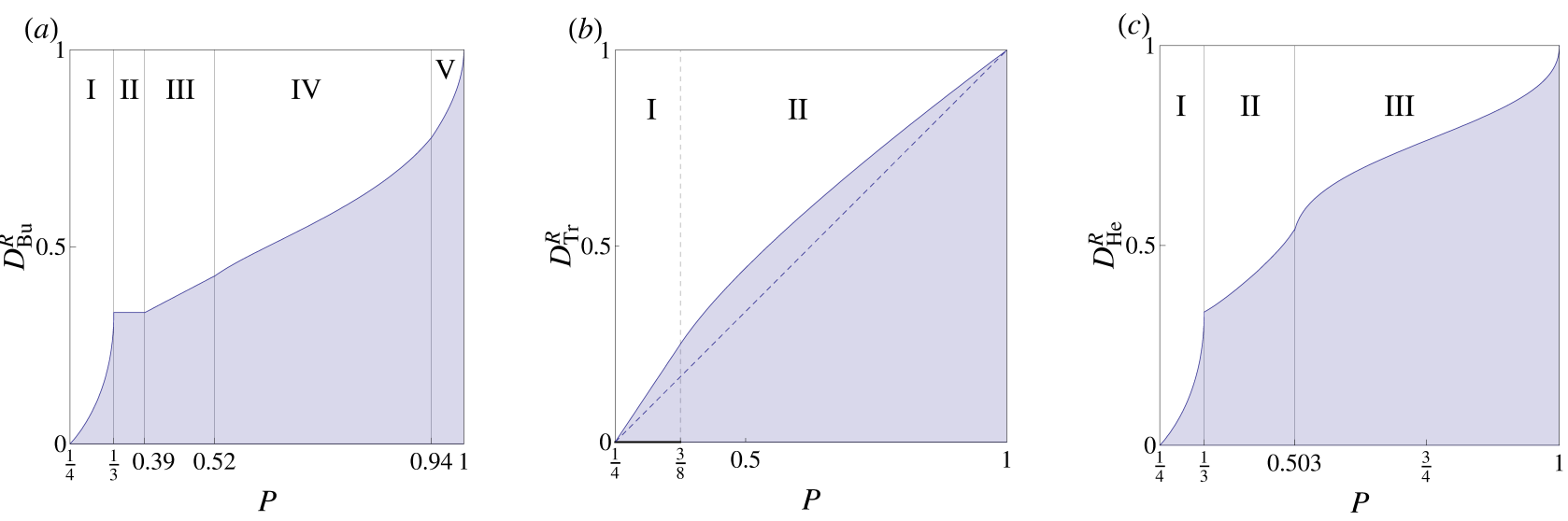

FIG. 4: (a) Bures discord of response accessible for the two-qubit states $\varrho$ with purity $P=\operatorname{Tr} \varrho^{2}$ (from Ref. 11]). The possible values of $D_{\mathrm{Bu}}^{R}(\varrho)$ as $P$ varies between $1 / 4$ and 1 are represented by the shadowed region. The solid line bounding this region on its upper side gives the discord of response of the maximally quantum-correlated states with respect to $D_{\mathrm{Bu}}^{R}$. These states are determined explicitly in Ref. [11] and have different forms in the five regions (I)-(V) delimited by the vertical lines. (b) Same for the trace discord of response $D_{\mathrm{Tr}}^{R}$. The values of $D_{\mathrm{Tr}}^{R}$ for the Werner states are represented here by the dashed line (these values are the same for the two branches in Eq. (113) for $P \in[1 / 4,1 / 3])$. The maximally quantum-correlated states are the Bell-diagonal states defined in Eqs. (C1) and (C2) in Appendix C for the two regions (I) and (II) delimited by the vertical line. (c) Same for the Hellinger discord of response $D_{\mathrm{He}}^{R}$. The maximally quantum-correlated states are characterized in Appendix

\section{MAXIMALLY QUANTUM-CORRELATED STATES}

In this Section we study the maximally quantum-correlated states with respect to various discords of response. With the help of numerical investigations, we determine the two-qubit states $\varrho$ with a fixed purity $P=\operatorname{Tr} \varrho^{2}$ having the highest trace discord of response and Hellinger discord of response. In this way, we complete the previous analysis carried out in Refs. 13] and [11], respectively for $D_{\mathrm{HS}}^{R}$ and $D_{\mathrm{Bu}}^{R}$. We find that the four discords of response yield different families of maximally quantum-correlated states with fixed purity $P<1$. Nevertheless, if $P$ is not fixed, $D_{\mathrm{Tr}}^{R}, D_{\mathrm{Bu}}^{R}$, and $D_{\mathrm{He}}^{R}$ take their maximal value (equal to unity) for the same class of states, namely the maximally entangled states, in agreement with Axiom (v) of Section [.

\section{A. States with a fixed purity maximizing the discords of response}

We restrict here our analysis to the case of two qubits $A$ and $B$ and use a numerical approach. We have computed the values of $D_{\mathrm{Tr}}^{R}, D_{\mathrm{Bu}}^{R}$, and $D_{\mathrm{He}}^{R}$ for $3 \times 10^{5}$ randomly generated two-qubit states, whose eigenvalues are chosen randomly with a uniform distribution (with the constraint that they are non-negative and sum up to unity) and eigenvectors are the column vectors of a random unitary matrix distributed according to the Haar measure. We identify among these random states those with purity $P$ maximizing the various discords of response. These families of most discordant states are tested by applying small disturbance analysis.

Our numerical analysis indicates that the states $\varrho_{\max }^{P}$ with purity $P$ maximizing the trace discord of response are given by Eqs. (C1) and (C2) in Appendix C] Since these states belong to the class of Bell-diagonal states, their trace geometric discord can be evaluated by using the results of Ref. 21] and one can take advantage of $D_{\mathrm{Tr}}^{R}=D_{\mathrm{Tr}}^{G}$ (recall that we are considering two qubits) to obtain:

Conjecture 1. The maximal value of $D_{\operatorname{Tr}}^{R}(\varrho)$ for all two-qubit states $\varrho$ with purity $P=\operatorname{Tr} \varrho^{2}$ is given by the following function of $P$ :

$$
D_{\operatorname{Tr}}^{R}\left(\varrho_{\max }^{P}\right)= \begin{cases}\frac{1}{2}(4 P-1) & \text { if } 1 / 4 \leq P \leq 3 / 8 \\ \frac{1}{9}(\sqrt{6 P-2}+1)^{2} & \text { if } 3 / 8 \leq P \leq 1\end{cases}
$$

This result, which relies on our conjecture for the maximally quantum-correlated states with respect to $D_{\mathrm{Tr}}^{R}$, Eqs. (C1) and (C2), is derived for completeness in Appendix C without relying on the results of Ref. [21]. One can find in a similar way the values of $D_{\mathrm{Tr}}^{R}$ for the Werner states

$$
\varrho_{\mathrm{W}}=a_{ \pm} \frac{\mathbb{1}}{4}+\left(1-a_{ \pm}\right)\left|\Psi_{-}\right\rangle\left\langle\Psi_{-}\right| \quad, \quad a_{ \pm}=\left\{\begin{array}{ll}
1+\sqrt{(4 P-1) / 3} & \text { for } P \in[1 / 4,1 / 3] \\
1-\sqrt{(4 P-1) / 3} & \text { for } P \in[1 / 4,1]
\end{array},\right.
$$


(a)

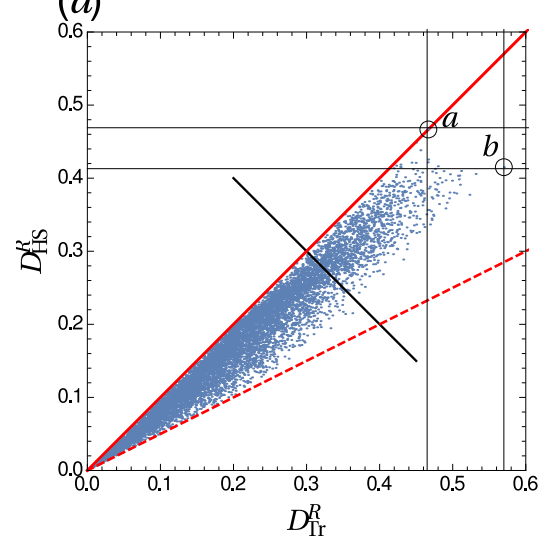

(b)

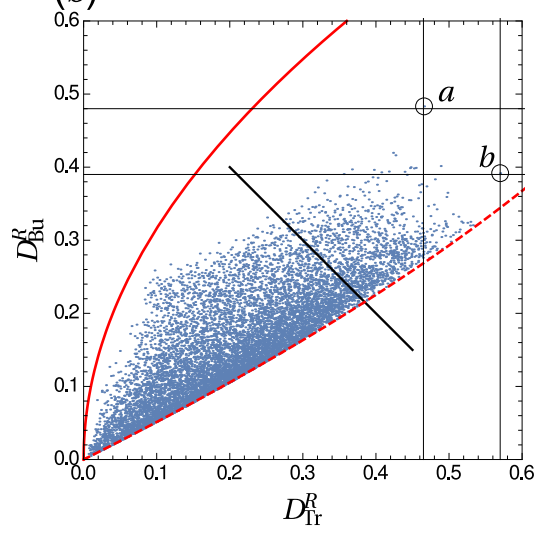

$(c)$

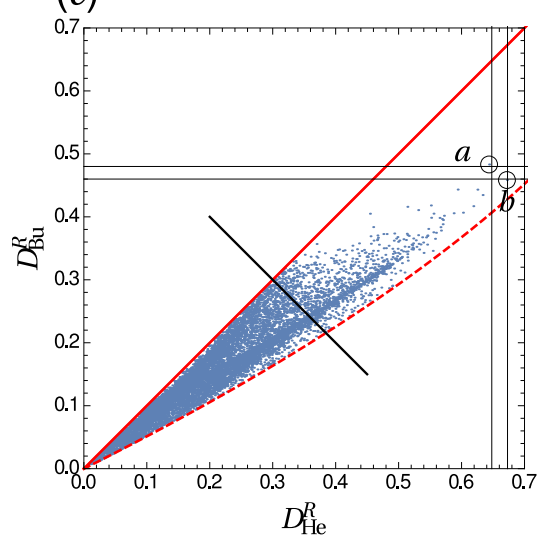

FIG. 5: (a) Values of the Hilbert-Schmidt and trace discords of response $D_{\mathrm{HS}}^{R}$ and $D_{\mathrm{Tr}}^{R}$ for $10^{4}$ random two-qubit states of different ranks with the same fixed global state purity $P=0.6$. The points $a$ and $b$ represent, respectively, some maximally quantum-correlated states with purity $P$ with respect to $D_{\mathrm{HS}}^{R}$ and $D_{\mathrm{Tr}}^{R}$. Note that $a$ is not maximally quantum correlated with respect to $D_{\mathrm{Tr}}^{R}$, and similarly for $b$ and $D_{\mathrm{HS}}^{R}$. The red solid and dashed lines represent the bounds discussed in Sec. IID like in Fig. 1. States on the thick black line have a hierarchy with respect to $D_{\mathrm{HS}}^{R}$ that is reversed compared to the hierarchy with respect to $D_{\mathrm{Tr}}^{R}$. (b) Same for the Bures and trace discords of response $D_{\mathrm{Bu}}^{R}$ and $D_{\mathrm{Tr}}^{R}$. (c) Same for the Bures and Hellinger discords of response $D_{\mathrm{Bu}}^{R}$ and $D_{\mathrm{He}}^{R}$.

where $\left|\Psi_{-}\right\rangle=(|01\rangle-|10\rangle) / \sqrt{2}$ denotes the Bell state. The Werner states maximize the Hilbert-Schmidt discord of response at fixed purity $P$ (for $P \in[1 / 4,1 / 3]$ this is true for the two branches of Werner states in Eq. (113), which yield to the same value of $\left.D_{\mathrm{HS}}^{R}\left(\varrho_{\mathrm{W}}\right)\right)$, see Ref. [13]. We display in panel (b) of Fig. 团 the accessible values of $D_{\mathrm{Tr}}^{R}(\varrho)$ as a function of $P$ (shadowed region). We clearly see in this figure that if $P$ is distinct from the smallest and highest possible purities $P=1 / 4$ and $P=1$ (i.e., if $\varrho$ is neither a maximally mixed nor a pure state), the trace discord of response of the Werner state (dashed line) is below the maximal value of $D_{\operatorname{Tr}}^{R}$ given by Eq. (112). This shows that the maximally quantum-correlated states with fixed purity $P$ are different for the two measures $D_{\mathrm{Tr}}^{R}$ and $D_{\mathrm{HS}}^{R}$.

A similar statement holds for the other discords of response. The maximal values of $D_{\mathrm{Bu}}^{R}$ and $D_{\mathrm{He}}^{R}$ at fixed purity $P$ cannot be characterized by such simple functions as in Eq. (112), therefore we do not report them here. The maximal Bures discord of response has been determined explicitly as a function of $P$ in Ref. [11] and is shown in panel (a) of Fig. [4. By using the numerical method described above, we have identified the two-qubit states with purity $P$ maximizing the Hellinger discord of response, which are given in Appendix $\mathrm{C}$ From the analytical expression given in Eq. (22), it is then easy to compute numerically the maximal Hellinger discord of response as a function of $P$. The latter is represented by the upper solid line in panel (c) of Fig. 4.

In Fig. 15 we report the distributions in the planes defined by pairs of discords of response associated to different distances for random two-qubit states. These distributions are analogous to those of Fig. 10xcept that they correspond here to a fixed purity $P=0.6$. Random states with this purity are generated as described above: their eigenvectors are obtained from random unitary matrices distributed according to the Haar measure, while their eigenvalues are picked randomly from the set of non-negative numbers $p_{i}$ with fixed sums $\sum_{i} p_{i}=1$ and $\sum_{i} p_{i}^{2}=0.6$. For states of rank $r>2$, we first generate $r-2$ random eigenvalues with a uniform distribution on sufficiently small intervals. The remaining eigenvalues are given by the constraints on the trace and the purity.

Since there is no exact relation between the discords of response associated to different distances, the points in Fig. are not distributed on a line but in a region of the plane with a non-vanishing area. This means that the different discords of response do not define the same ordering on the set of bipartite states: for instance, it is possible to find two states $\varrho_{1}$ and $\varrho_{2}$ which satisfy $D_{\operatorname{Tr}}^{R}\left(\varrho_{1}\right)<D_{\operatorname{Tr}}^{R}\left(\varrho_{2}\right)$ and at the same time $D_{\mathrm{Bu}}^{R}\left(\varrho_{1}\right)>D_{\mathrm{Bu}}^{R}\left(\varrho_{2}\right)$ (see e.g. the points lying on the thick line in Fig. F(b)). In other words, changing the distance modifies the ordering of the states. In Fig. [5 the different locations of the points having the highest discord of response for the different distances illustrate the fact that the maximally quantum-correlated states with purity $P<1$ are not the same for $D_{\mathrm{Tr}}^{R}, D_{\mathrm{Bu}}^{R}$, and $D_{\mathrm{He}}^{R}$.

We have observed a similar behavior as in Fig. 15 for the trace, Bures, and Hellinger measurement-induced geometric discords (not reported here).

\section{B. States with arbitrary purity maximizing the discords of response}

In spite of the annoying fact that the maximally quantum-correlated states with a fixed purity depend on the distance used to define the discord of response, a universal family of states emerges when one considers the maximal 
value of $D^{R}$ irrespective of the purity $P$. As it should be expected for any proper measure of quantum correlations, for the trace, Bures, and Hellinger distances these maximally discordant states are the maximally entangled states, that is, the states $\varrho$ with largest entanglement of formation $E_{\mathrm{EoF}}(\varrho)=\ln \left(\min \left\{n_{A}, n_{B}\right\}\right)$. Let us recall that if $n_{A} \leq n_{B}$, such states are convex combinations of pure states of the form:

$$
\left|\Psi_{k}\right\rangle=\frac{1}{\sqrt{n_{A}}} \sum_{i=1}^{n_{A}}\left|\varphi_{k i}\right\rangle\left|\chi_{k i}\right\rangle
$$

with $\left\langle\varphi_{k i} \mid \varphi_{k j}\right\rangle=\delta_{i j}$ and $\left\langle\chi_{k i} \mid \chi_{l j}\right\rangle=\delta_{i j} \delta_{k l}$ (see, e.g., Ref. [15], Proposition 9.E.1). Notice that the subspaces $\operatorname{span}\left\{\left|\chi_{k i}\right\rangle, i=1, \ldots, n_{A}\right\}$ are orthogonal for different $k$ 's, so that the aforementioned convex combinations involve at most $r$ pure states $\left|\Psi_{k}\right\rangle$ if $r n_{A} \leq n_{B}<(r+1) n_{A}$. In particular, if $n_{A} \leq n_{B}<2 n_{A}$ then the maximally entangled states are necessarily pure states given by Eq. (114). The following theorem is proven in Appendix B]

Theorem 13. Let subsystems $A$ and $B$ have arbitrary space dimensions $n_{A}$ and $n_{B}$, with $n_{A} \leq n_{B}$. Then the maximal value of the trace, Bures, and Hellinger discords of response is equal to unity and these three discords of response satisfy Axiom (v) of Section $\square$, namely, $D^{R}(\varrho)=1$ if and only if $E_{\mathrm{EoF}}(\varrho)=\ln n_{A}$. In contrast, if $2 n_{A} \leq n_{B}$ then the Hilbert-Schmidt discord of response does not enjoy this property.

It is shown in Appendix B that the trace, Bures, and Hellinger geometric discords and measurement-induced geometric discords satisfy Axiom (v) as well, at least when $A$ is a qubit, as reported in Table 11 (see also Theorem 6 ).

\section{QUANTUMNESS BREAKING CHANNELS}

Quantum channels (also called quantum operations) are by definition dynamical maps on the set of quantum states which are completely positive $(\mathrm{CP})$ and trace-preserving $(\mathrm{TP})$. Let us recall that a linear map $\Phi_{A}$ acting on the set of states of a system $A$ is CP if its trivial extensions $\Phi_{A} \otimes \mathbb{1}_{B}$ are positive (i.e., they transform non-negative matrices into non-negative matrices) for any system $B$ with finite-dimensional Hilbert space $\mathcal{H}_{B}$. A $\mathrm{TP}$ linear map $\Phi_{A}$ is $\mathrm{CP}$ (and hence is a quantum channel) if and only if [37]

$$
\varrho^{\Phi_{A}} \equiv \Phi_{A} \otimes \mathbb{1}_{B}\left(\left|\Psi_{+}\right\rangle\left\langle\Psi_{+}\right|\right)
$$

is non-negative. Here, we have introduced a system $B$ having the same space dimension $n_{B}=n_{A}$ as $A$, a fixed orthonormal product basis $\left\{\left|i_{A}, j_{B}\right\rangle \equiv|i\rangle_{A} \otimes|j\rangle_{B}\right\}_{i, j=1}^{n_{A}}$ for the composite system $A B$, and the maximally entangled state $\left|\Psi_{+}\right\rangle=\frac{1}{\sqrt{n_{A}}} \sum_{i=1}^{n_{A}}\left|i_{A}, i_{B}\right\rangle$ of $A B$. The state $\varrho^{\Phi_{A}}$ is called the Jamiołkowski state corresponding to the CPTP $\operatorname{map} \Phi_{A}$.

In Ref. [68], the authors have characterized the local quantum channels $\Phi_{A}$ acting on system $A$ that destroy all the quantum correlations existing in an arbitrary bipartite quantum state of $A B$. Such channels are called quantumness breaking channels and are such that the output state $\Phi_{A} \otimes \mathbb{1}_{B}\left(\varrho_{A B}\right)$ is a classical-quantum state for any bipartite input state $\varrho_{A B}$. It turns out that a channel $\Phi_{A}$ is quantumness breaking if and only if its Jamiołkowski state $\varrho^{\Phi_{A}}$ is classical-quantum (see Ref. [68]).

In this Section, we derive a user-friendly, necessary condition for a channel to be quantumness breaking. This condition is formulated in terms of the rank of the superoperator $\widehat{\Phi}_{A}$ associated to the quantum channel $\Phi_{A}$. This superoperator is defined as the operator on the tensor-product (doubled) Hilbert space $\mathcal{H}_{A} \otimes \mathcal{H}_{A}$ with matrix elements

$$
\left\langle i_{A}, j_{A}\left|\widehat{\Phi}_{A}\right| k_{A}, l_{A}\right\rangle \equiv\left\langle i_{A}\left|\Phi_{A}\left(\left|k_{A}\right\rangle\left\langle l_{A}\right|\right)\right| j_{A}\right\rangle .
$$

If we represent the states $\varrho_{A}$ of $A$ as vectors $\left|\varrho_{A}\right\rangle$ on $\mathcal{H}_{A} \otimes \mathcal{H}_{A}$ with components $\left\langle i_{A}, j_{A} \mid \varrho_{A}\right\rangle=\left\langle i_{A}\left|\varrho_{A}\right| j_{A}\right\rangle$, then $\widehat{\Phi}_{A}$ realizes the transformation of these vector-states under the channel $\Phi_{A}$.

Our treatment relies on the so-called reshuffling operation $\mathcal{R}$, which is a widely employed tool in the theory of quantum channels, see, e.g., Ref. [37]. The operation $\mathcal{R}$ exchanges the matrix entries of a block matrix in the following way: given an operator $X$ on $\mathcal{H}_{A} \otimes \mathcal{H}_{B}$, one associates to it the operator $X^{\mathcal{R}}$ from $\mathcal{H}_{B} \otimes \mathcal{H}_{B}$ to $\mathcal{H}_{A} \otimes \mathcal{H}_{A}$ with matrix elements

$$
\left\langle i_{A}, j_{A}\left|X^{\mathcal{R}}\right| k_{B}, l_{B}\right\rangle \equiv\left\langle i_{A}, k_{B}|X| j_{A}, l_{B}\right\rangle \text {. }
$$

Thus, the reshuffling operation transforms a $n_{A} n_{B} \times n_{A} n_{B}$ matrix onto a $n_{A}^{2} \times n_{B}^{2}$ matrix and vice versa. It provides a connection between the superoperator $\widehat{\Phi}_{A}$ associated to the quantum channel $\Phi_{A}$ and the corresponding Jamiołkowski state thanks to the following relation [37]:

$$
\left(\widehat{\Phi}_{A}\right)^{\mathcal{R}}=n_{A} \varrho^{\Phi_{A}}
$$

Our necessary condition for a channel to be quantumness breaking is based on the following theorem: 
Theorem 14. For any state $\varrho$ of a bipartite quantum system $A B$, the Hilbert-Schmidt geometric discord $D_{\mathrm{HS}}^{G}(\varrho)$ is bounded from below in the following fashion:

$$
D_{\mathrm{HS}}^{G}(\varrho) \geq \mu_{n_{A}+1}+\ldots+\mu_{n_{A}^{2}},
$$

where $\mu_{1} \geq \cdots \geq \mu_{n_{A}^{2}}$ are the squared singular values of the reshuffled density matrix $\varrho^{\mathcal{R}}$ in decreasing order.

Recall that the squared singular values of $\varrho^{\mathcal{R}}$ are the eigenvalues $\mu_{i}$ of the $n_{A}^{2} \times n_{A}^{2}$ matrix $\varrho^{\mathcal{R}}\left(\varrho^{\mathcal{R}}\right)^{\dagger}$. In Appendix $\mathrm{D}$ we prove that the inequality in Eq. (119) turns into an equality provided that party $A$ is a qubit and $\varrho$ has maximally mixed marginals $\varrho_{A}=\mathbb{1} / 2$ and $\varrho_{B}=\mathbb{1} / n_{B}$. A bound similar to that of Eq. [119) has been derived in Ref. 69], where it was also found that this bound is saturated for Bell-diagonal states.

It is worthwhile mentioning that the singular values of $\varrho^{\mathcal{R}}$ appear in the generalized Schmidt decomposition of mixed states. Given an arbitrary density matrix $\varrho$ on $\mathcal{H}_{A} \otimes \mathcal{H}_{B}$, this decomposition reads (see, e.g., Refs. 15, 37])

$$
\varrho=\sum_{m=1}^{n_{A}^{2}} \sqrt{\mu_{m}} X_{m} \otimes Y_{m},
$$

where $\left\{X_{m}\right\}_{m=1}^{n_{A}^{2}}$ and $\left\{Y_{p}\right\}_{p=1}^{n_{B}^{2}}$ are orthonormal bases of the Hilbert spaces formed by all $n_{A} \times n_{A}$ matrices and all $n_{B} \times n_{B}$ matrices, respectively (i.e., $\operatorname{Tr}_{A}\left(X_{m}^{\dagger} X_{n}\right)=\delta_{m n}$ and $\left.\operatorname{Tr}_{B}\left(Y_{p}^{\dagger} Y_{q}\right)=\delta_{p q}\right)$ and we have assumed $n_{A} \leq n_{B}$. The matrices $X_{m}$ and $Y_{p}$ are given in terms of the eigenvectors of $\varrho^{\mathcal{R}}\left(\varrho^{\mathcal{R}}\right)^{\dagger}$ and $\left(\varrho^{\mathcal{R}}\right)^{\dagger} \varrho^{\mathcal{R}}$, respectively. Note that $\sum_{m} \mu_{m}$ coincides with the state purity $P=\operatorname{Tr} \varrho^{2}$. Moreover, $\sum_{m} \sqrt{\mu_{m}}>1$ is a sufficient (but not necessary) condition for entanglement [70]. Analogously, it follows from Theorem 14 that $\sum_{m>n_{A}} \mu_{m}>0$ is a sufficient condition for $\varrho$ to be quantum correlated. Indeed, although $D_{\mathrm{HS}}^{G}$ is not a proper measure of quantum correlations, it satisfies Axiom (i) of Section \(see Sec. IIIC).

In view of the relation of Eq. (118) and of the aforementioned characterization of quantumness breaking channels in Ref. [68], one deduces from Theorem 14 the following result:

Corollary 2. If the rank of $\widehat{\Phi}_{A}$ is larger than $n_{A}$, then the channel $\Phi_{A}$ is not quantumness breaking.

Theorem 14 actually provides a quantitative estimate which can be used to discriminate channels that are not quantumness breaking, since it gives a lower bound on the minimal amount of quantum correlations that survive after the action of a local quantum channel $\Phi_{A}$ if the input state is the maximally entangled state $\left|\Psi_{+}\right\rangle$. Such residual amount, as measured e.g. by the trace geometric discord $D_{\operatorname{Tr}}^{G}$, cannot be smaller than the sum of the $n_{A}^{2}-n_{A}$ smallest squared singular values of $\widehat{\Phi}_{A} / n_{A}$ (recall that $D_{\mathrm{Tr}}^{G} \geq D_{\mathrm{HS}}^{G}$, see Eq. (15)).

Proof of Theorem 14. Since the reshuffling procedure consists only in exchanging matrix entries, it neither changes the Hilbert-Schmidt norm of a matrix, nor the Hilbert-Schmidt distance between two matrices which are both reshuffled by $\mathcal{R}$. Observe that the reshuffling operation transforms a classical-quantum state $\sigma_{\text {cq }}$ into a matrix of rank equal to the dimensionality $n_{A}$ of subsystem $A$. More precisely, consider a classical-quantum state $\sigma_{\mathrm{cq}}$ given by Eq. (1). Then $\Pi \sigma_{\mathrm{cq}}^{\mathcal{R}}=\sigma_{\mathrm{cq}}^{\mathcal{R}}$, where $\Pi$ is the projector of rank $n_{A}$ defined by $\Pi=\sum_{i}\left|\alpha_{i}\right\rangle\left\langle\alpha_{i}|\otimes| \bar{\alpha}_{i}\right\rangle\left\langle\bar{\alpha}_{i}\right|$ (the bars denote complex conjugation in the standard basis, i.e., $\left\langle j_{A} \mid \bar{\alpha}_{i}\right\rangle=\left\langle j_{A} \mid \alpha_{i}\right\rangle^{*}$ for any $\left.j_{A}=1, \ldots, n_{A}\right)$. We will now estimate the geometric discord $D_{\mathrm{HS}}^{G}(\varrho)$ by the Hilbert-Schmidt distance from $\varrho^{\mathcal{R}}$ to the nearest $n_{A}^{2} \times n_{B}^{2}$ matrix $M_{n_{A}}$ of rank $n_{A}$ :

$$
D_{\mathrm{HS}}^{G}(\varrho)=\min _{\sigma_{\mathrm{cq}}}\left\|\varrho-\sigma_{\mathrm{cq}}\right\|_{\mathrm{HS}}^{2} \geq \min _{M_{n_{A}}}\left\|\varrho^{\mathcal{R}}-M_{n_{A}}\right\|_{\mathrm{HS}}^{2} .
$$

On the other hand, by the low-rank approximation theorem (see, e.g., Ref. [71]) one has

$$
\begin{aligned}
\left\|\varrho^{\mathcal{R}}-M_{n_{A}}\right\|_{\mathrm{HS}}^{2} & =\operatorname{Tr}\left(\varrho^{\mathcal{R}}-M_{n_{A}}\right)^{\dagger} \Pi\left(\varrho^{\mathcal{R}}-M_{n_{A}}\right)+\operatorname{Tr}\left(\varrho^{\mathcal{R}}-M_{n_{A}}\right)^{\dagger}(1-\Pi)\left(\varrho^{\mathcal{R}}-M_{n_{A}}\right) \\
& =\left\|\Pi \varrho^{\mathcal{R}}-M_{n_{A}}\right\|_{\mathrm{HS}}^{2}+\operatorname{Tr} \varrho^{\mathcal{R}}\left(\varrho^{\mathcal{R}}\right)^{\dagger}(1-\Pi) \\
& \geq \operatorname{Tr} \varrho^{\mathcal{R}}\left(\varrho^{\mathcal{R}}\right)^{\dagger}(1-\Pi) \geq \mu_{n_{A}+1}+\cdots+\mu_{n_{A}^{2}},
\end{aligned}
$$

where $\Pi$ is the projector of rank $n_{A}$ on the range of $M_{n_{A}}$ and $\mu_{m}$ are the eigenvalues of $\varrho^{\mathcal{R}}\left(\varrho^{\mathcal{R}}\right)^{\dagger}$ (i.e., the squared singular values of $\varrho^{\mathcal{R}}$ ) in decreasing order. The last inequality comes from the min-max principle (see, e.g., Ref. [64]). Comparing Eqs. (121) and (122), the desired bound of Eq. (119) follows. Note that, according to Eq. (122), this inequality turns into an equality if $\Pi^{\mathrm{opt}} \varrho^{\mathcal{R}}=\sigma_{\mathrm{HS}, \varrho}^{\mathcal{R}}$, where $\Pi^{\mathrm{opt}}$ is the projector on the sum of the eigenspaces associated to the largest $n_{A}$ eigenvalues of $\varrho^{\mathcal{R}}\left(\varrho^{\mathcal{R}}\right)^{\dagger}$ and $\sigma_{\mathrm{HS}, \varrho}$ is the closest classical-quantum state to $\varrho$ (as measured by the Hilbert-Schmidt distance), which is given by Eq. (172). 


\section{DISCUSSION AND CONCLUSIONS}

One of the most relevant results of the present study is that the Hellinger geometric discord $D_{\mathrm{He}}^{G}$ and the Hellinger discord of response $D_{\mathrm{He}}^{R}$ provide the first instance of bona fide measures of quantum correlations which are at the same time easy to compute (see Eqs. (20) and (22)), satisfy all the axiomatic criteria for proper measures of quantum discord given in the Introduction, and enjoy clear operational interpretations in quantum protocols. They thus satisfy all the fundamental requirements of computability, reliability, and operational viability.

Let us briefly discuss the operational interpretations of these two measures and of the other geometric measures of quantum discord studied in this work. If the reference subsystem $A$ is a qubit, the Hellinger discord of response coincides with the local quantum uncertainty. The latter indeed enjoys a simple operational interpretation described in Sec. III Thanks to the relation between $D_{\mathrm{He}}^{R}$ and $D_{\mathrm{He}}^{G}$ provided by Eq. (95), the same holds for the Hellinger geometric discord. Besides this interpretation, $D_{\mathrm{He}}^{R}$ and $D_{\mathrm{He}}^{G}$ enjoy further operational meanings in terms of the minimum probability of error in discriminating two equiprobable quantum states if infinitely many copies can be used to distinguish them [46].

In a one-shot scenario, the minimum probability of error in discriminating two equiprobable states $\varrho_{1}$ and $\varrho_{2}$ is given in terms of the trace norm $\left\|\varrho_{2}-\varrho_{1}\right\|_{\operatorname{Tr}}$ according to the Helstrom formula [55], see Eq. (52). This formula grants an operational meaning to all geometric measures of quantum discord defined with the trace distance, for instance in the context of quantum reading [32]. In this protocol [72], the task is to distinguish the output states of a quantum transmitter which goes through an unknown binary cell changing the transmitter states. If the actions of the binary cells on these states are given by the identity and local unitary transformations with a harmonic spectrum, the minimum probability of error maximized over all possible realizations of the cells is a simple function of the trace discord of response $D_{\operatorname{Tr}}^{R}$. As we have shown in the present work, if subsystem $A$ is a qubit then $D_{\operatorname{Tr}}^{R}$ coincides with the trace geometric discord $D_{\mathrm{Tr}}^{G}$ and the trace measurement-induced geometric discord $D_{\mathrm{Tr}}^{M}$. Hence, also these last two measures enjoy direct operational interpretations. Moreover, as shown in Ref. [32], the minimum probability of error in quantum reading features tight lower and upper bounds that are simple functions of the Bures and Hellinger discords of response $D_{\mathrm{Bu}}^{R}$ and $D_{\mathrm{He}}^{R}$.

Furthermore, the Bures geometric discord $D_{\mathrm{Bu}}^{G}$ enjoys a simple operational interpretation in terms of the minimum probability of error in the task of discriminating selected states within a given ensemble, as discussed in Ref. [9]. Due to the relation obtained above between $D_{\mathrm{Bu}}^{G}$ and $D_{\mathrm{Bu}}^{R}$ when party $A$ is a qubit, see Eq. (102), this operational interpretation extends also to the Bures discord of response $D_{\mathrm{Bu}}^{R}$.

For the sake of completeness, we should also mention some further instances of active research fields of quantum technologies in which geometric measures of quantum correlations find interesting applications. These include protocols such as quantum metrology with unknown disturbance [73], quantum illumination [28], and entanglement distribution between system and apparatus during a measurement process 74 .

In conclusion, we have investigated the main properties of different classes of geometric measures of quantum correlations. We have characterized, quantified, and compared them for the most significant contractive distances (trace, Bures, and Hellinger distances) and operations (geometric discord, measurement-induced geometric discord, and discord of response). We have proven a variety of bounds and algebraic relations between these geometric measures. The main results are summarized in the synoptic tables of Section II (see Tables IIII). Thanks to the one-to-one correspondence that we have established between some of these measures, one can extend the physical interpretation from one class of measures to other classes that are in direct correspondence with the former. We have found that direct one-to-one correspondences exist only in the case of low-dimensional reference subsystems (qubit or qutrit). Otherwise, in more general situations, we have established a substantial set of inequalities, some of them being tight.

We have also established that different geometric measures of quantum discord induce in general different orderings of the discordant states. This phenomenon is quite analogous to the different ordering of quantum states established by different entanglement measures [75, 76]. In particular, different measures of quantum discord identify different classes of maximally quantum-correlated states with a fixed purity. On the other hand, the set of maximally quantumcorrelated states with arbitrary purity is independent of the choice of the distances and operations, and coincides with the set of maximally entangled states.

Finally, we have established a useful role also for the Hilbert-Schmidt geometric discord $D_{\text {HS }}^{G}$, notwithstanding that it is strictly speaking not a measure of quantum correlations due to the fact that the Hilbert-Schmidt distance is not contractive under CPTP maps. Indeed, $D_{\mathrm{HS}}^{G}$ provides useful bounds on bona fide geometric measures based on contractive distances. Furthermore, we have exploited this fact in order to determine a necessary condition for local quantum channels to be quantumness breaking, namely, to destroy all quantum correlations featured by arbitrary input states. This condition can be formulated in terms of the Jamiołkowski state that corresponds to the given channel. 


\section{Acknowledgments}

F.I. and W.R. acknowledge the EU FP7 Cooperation STREP Projects iQIT - integrated Quantum Information Technologies, Grant Agreement No. 270843, and EQuaM - Emulators of Quantum Frustrated Magnetism, Grant Agreement No. 323714. They also acknowledge financial support from the Italian Minister of Scientific Research (MIUR) under the national PRIN programme. D.S. acknowledges financial support from the ANR project no. ANR13-JS01-0005-01. We are grateful to S. Rana for pointing us the results of Ref. [16] on the trace measurement-induced geometric discord.

\section{Appendix A: Proofs of Theorems 8 and 12 of Section VI}

\section{Proof of Theorem 8 and of some inequalities in Table III}

We first consider the Hilbert-Schmidt discord of response. We will afterward make use of the relations Eq. (17) to deduce the statements on $D_{\mathrm{He}}^{R}$ in Theorem 8 . According to the definition of $D^{R}$ in Eq. (7), one has:

$$
2 D_{\mathrm{HS}}^{R}(\varrho)=\min _{U_{A} \in \mathcal{U}_{\Lambda}}\left\|\varrho-U_{A} \otimes \mathbb{1} \varrho U_{A}^{\dagger} \otimes \mathbb{1}\right\|_{\mathrm{HS}}^{2},
$$

the minimum being over all unitaries $U_{A}$ acting on subsystem $A$ with spectrum given by the complex roots of unity. Any such unitary operator has the form given in Eq. (100). Therefore,

$$
2 D_{\mathrm{HS}}^{R}(\varrho)=\min _{\left\{\left|\alpha_{i}\right\rangle\right\}} \| \sum_{i, j=1}^{n_{A}}\left(1-e^{-\mathrm{i} \frac{2 \pi(i-j)}{n_{A}}}\right)\left|\alpha_{i}\right\rangle\left\langle\alpha_{j}\right| \otimes\left\langle\alpha_{i}|\varrho| \alpha_{j}\right\rangle \|_{\mathrm{HS}}^{2} .
$$

Now, the squared Hilbert-Schmidt norm of a block matrix is the sum of the squared Hilbert-Schmidt norms of each blocks, i.e., $\| \sum_{i, j}\left|\alpha_{i}\right\rangle\left\langle\alpha_{j}\right| \otimes X_{i j}\left\|_{\mathrm{HS}}^{2}=\sum_{i, j}\right\| X_{i j} \|_{\mathrm{HS}}^{2}$. This yields the relation

$$
D_{\mathrm{HS}}^{R}(\varrho)=2 \min _{\left\{\left|\alpha_{i}\right\rangle\right\}} \sum_{i \neq j} \sin ^{2}\left(\frac{\pi(i-j)}{n_{A}}\right)\left\|\left\langle\alpha_{i}|\varrho| \alpha_{j}\right\rangle\right\|_{\mathrm{HS}}^{2} .
$$

Similarly, the Hilbert-Schmidt measurement-induced geometric discord takes the following expression:

$$
D_{\mathrm{HS}}^{M}(\varrho)=\min _{\left\{\left|\alpha_{i}\right\rangle\right\}} \sum_{i \neq j}\left\|\left\langle\alpha_{i}|\varrho| \alpha_{j}\right\rangle\right\|_{\mathrm{HS}}^{2}
$$

By comparing Eqs. and at at we obtain that

$$
D_{\mathrm{HS}}^{R}(\varrho)= \begin{cases}2 D_{\mathrm{HS}}^{M}(\varrho)=2 D_{\mathrm{HS}}^{G}(\varrho) & \text { if } n_{A}=2 \\ \frac{3}{2} D_{\mathrm{HS}}^{M}(\varrho)=\frac{3}{2} D_{\mathrm{HS}}^{G}(\varrho) & \text { if } n_{A}=3\end{cases}
$$

where we have made use of the equality between the Hilbert-Schmidt geometric discord and the measurement-induced geometric discord (see Section IVB). For $n_{A}>3$, comparing Eqs. (A3) and (A4) and observing that

$$
\sin ^{2}\left(\frac{\pi}{n_{A}}\right) \leq \sin ^{2}\left(\frac{\pi(i-j)}{n_{A}}\right) \leq 1 \quad, \quad i, j=1, \ldots, n_{A}, i \neq j
$$

we obtain the following inequalities

$$
2 \sin ^{2}\left(\frac{\pi}{n_{A}}\right) D_{\mathrm{HS}}^{G}(\varrho) \leq D_{\mathrm{HS}}^{R}(\varrho) \leq 2 D_{\mathrm{HS}}^{G}(\varrho)
$$

The above bounds remain valid for unnormalized non-negative operators $\varrho$. One may thus replace $\varrho$ by $\sqrt{\varrho}$ in Eq. (A77). In view of the relations between $D_{\mathrm{He}}^{R}$ and $D_{\mathrm{HS}}^{R}$ and between $D_{\mathrm{He}}^{G}$ and $D_{\mathrm{HS}}^{G}$ (see Theorem 2), this yields the inequalities given in Eq. (94). Similarly, the fundamental identities of Eq. (95) follow directly by substituting $\varrho$ by $\sqrt{\varrho}$ in Eq. (A5). This concludes the proof of Theorem 8 ,

Let us point out that the relation given in Eq. A5 between the Hilbert-Schmidt discord of response and the Hilbert-Schmidt geometric discord when $n_{A}=2$ has been found earlier in Ref. [13]. Moreover, the lower bound on $D_{\mathrm{HS}}^{R}(\varrho)$ in Eq. (A7]) is finer than a previously known bound [13]. 
Several additional results stated in the main text can be easily derived from the above considerations. Firstly, the general expression of the Hellinger discord of response in Eq. (10) is obtained by replacing $\varrho$ by its square root in Eq. A3 . Secondly, the bounds on the discord of response in terms of the geometric discord for the trace and Bures distances reported in Table [II are obtained by combining Eq. (A7) with the trivial bounds of Eqs. (15) and (16). More precisely, the bounds on $D_{\operatorname{Tr}}^{R}$ in terms of $D_{\operatorname{Tr}}^{G}$ for $n_{A} \geq 3$ are consequences of Eq. (A7), the two inequalities of Eq. (15), and the corresponding inequalities for the discord of response (with the correct factor of two coming from the normalization factor $\mathcal{N}$ in Eq. (7) $)$. The bounds on $D_{\mathrm{Bu}}^{R}$ in terms of $D_{\mathrm{Bu}}^{G}$ are deduced from the previous bounds on $D_{\operatorname{Tr}}^{R}$ by using Eqs. (16) and (41).

\section{Proof of Theorem 12}

We now turn to Theorem 12 on the trace discord of response. Indeed, $D_{\operatorname{Tr}}^{R}$ is expressed by a formula analogous to Eq. (A2) with the Hilbert-Schmidt norm replaced by the trace norm, excepted for a factor of 4 instead of 2 in the right-hand side. For $n_{A}=2$, setting $X_{12}=\left\langle\alpha_{1}|\varrho| \alpha_{2}\right\rangle$, the expression for $D_{\operatorname{Tr}}^{R}$ takes the form

$$
\begin{aligned}
D_{\operatorname{Tr}}^{R}(\varrho) & =\min _{\left\{\left|\alpha_{i}\right\rangle\right\}} \|\left|\alpha_{1}\right\rangle\left\langle\alpha_{2}\left|\otimes X_{12}+\right| \alpha_{2}\right\rangle\left\langle\alpha_{1}\right| \otimes X_{12}^{\dagger} \|_{\operatorname{Tr}}^{2}=\left(\operatorname{Tr} \sqrt{\left|\alpha_{1}\right\rangle\left\langle\left.\alpha_{1}|\otimes| X_{12}^{\dagger}\right|^{2}+\mid \alpha_{2}\right\rangle\left\langle\left.\alpha_{2}|\otimes| X_{12}\right|^{2}\right.}\right)^{2} \\
& =4\left\|X_{12}\right\|_{\operatorname{Tr}}^{2}
\end{aligned}
$$

where we have used the identity $\|X\|_{\operatorname{Tr}}=\left\|X^{\dagger}\right\|_{\operatorname{Tr}}$ in the last line. A similar calculation shows that the trace measurement-induced geometric discord is given by $D_{\operatorname{Tr}}^{M}(\varrho)=4\left\|X_{12}\right\|_{\operatorname{Tr}}^{2}$. Thus we arrive at the important equality $D_{\operatorname{Tr}}^{R}(\varrho)=D_{\operatorname{Tr}}^{M}(\varrho)$. Furthermore, it is proven in Ref. [21] that $D_{\operatorname{Tr}}^{G}(\varrho)=D_{\operatorname{Tr}}^{M}(\varrho)$ when $n_{A}=2$. Finally, the bound $D_{\operatorname{Tr}}^{R}(\varrho) \geq D_{\mathrm{HS}}^{R}(\varrho)$ follows from Eqs. (A3) and (A8) and from the trivial inequality $\|X\|_{\operatorname{Tr}} \geq\|X\|_{\mathrm{HS}}$.

\section{Appendix B: Geometric measures of quantum correlations satisfying Axiom (v)}

In this Appendix we show that the Bures, Hellinger, and trace discords of response satisfy Axiom (v) of Section 1 and that their maximum value equals unity, as stated in Theorem 13. We prove as well that the Hellinger geometric discord obeys Axiom (v) and its maximum value is $2-2 / \sqrt{n_{A}}$ for $n_{A}=2$ and $n_{A}=3$, as stated in Table III and we discuss the same issue for the other geometric measures of quantum discord.

Let us first focus on the discord of response $D^{R}$ for the Bures and Hellinger distances. For such distances it is obvious from the definitions, Eqs. (3), (4), and (7), that $D^{R} \leq 1$. Furthermore, $D^{R}(\varrho)=1$ if and only if for any local unitary operator $U_{A} \in \mathcal{U}_{\Lambda}$ with non-degenerate spectrum $\Lambda=\left\{\lambda_{1}, \ldots, \lambda_{n_{A}}\right\}$, one has that the Uhlmann fidelity $F\left(\varrho, U_{A} \otimes \mathbb{1} \varrho U_{A}^{\dagger} \otimes \mathbb{1}\right)=0$ for the Bures distance and $\operatorname{Tr} \sqrt{\varrho}\left(U_{A} \otimes \mathbb{1} \varrho U_{A}^{\dagger} \otimes \mathbb{1}\right)^{1 / 2}=0$ for the Hellinger distance. Each of these two conditions is equivalent to $\varrho$ and $U_{A} \otimes \mathbb{1} \varrho U_{A}^{\dagger} \otimes \mathbb{1}$ having orthogonal supports. This is in turn equivalent to the following statement: for any pair of eigenvectors $\left|\Psi_{k}\right\rangle$ and $\left|\Psi_{l}\right\rangle$ of $\varrho$ with nonzero eigenvalues, it holds that

$$
\operatorname{Tr}_{A}\left(D_{k l} U_{A}\right)=\left\langle\Psi_{l}\left|U_{A} \otimes \mathbb{1}\right| \Psi_{k}\right\rangle=0 \quad \forall U_{A} \in \mathcal{U}_{\Lambda}
$$

where we have set $D_{k l} \equiv \operatorname{Tr}_{B}\left(\left|\Psi_{k}\right\rangle\left\langle\Psi_{l}\right|\right)$.

We now argue that Eq. (B1) implies that $D_{k l}=\left(\delta_{k l} / n_{A}\right) \mathbb{1}_{A}$. Indeed, if $A$ is a self-adjoint matrix then

$$
\operatorname{Tr}(A U)=0 \quad \forall U \in \mathcal{U}_{\Lambda} \quad \Rightarrow \quad A=a \mathbb{1} \text { with } a \in \mathbb{R} .
$$

To verify that the implication in Eq. (B2) holds true, let us introduce a fixed orthonormal basis $\{|i\rangle\}$ of $\mathcal{H}_{A}$. We take $U_{t}=e^{-\mathrm{i} H t} U_{0} e^{\mathrm{i} H t}$ with $U_{0}=\sum_{i} \lambda_{i}|i\rangle\langle i|$ and $H$ self-adjoint. Then $U_{t} \in \mathcal{U}_{\Lambda}$ for any real $t$. Let $A$ be such that $\operatorname{Tr}(A U)=0$ for all $U \in \mathcal{U}_{\Lambda}$. Differentiating $\operatorname{Tr}\left(A U_{t}\right)=0$ with respect to $t$ and setting $t=0$, one obtains $\sum_{i} \lambda_{i}\langle i|[H, A]| i\rangle=0$. Choosing $H=e^{-\mathrm{i} \theta}\left|i_{0}\right\rangle\left\langle j_{0}\left|+e^{\mathrm{i} \theta}\right| j_{0}\right\rangle\left\langle i_{0}\right|$, this yields

$$
\left(\lambda_{i_{0}}-\lambda_{j_{0}}\right) \operatorname{Im}\left\{e^{\mathrm{i} \theta}\left\langle i_{0}|A| j_{0}\right\rangle\right\}=0 .
$$

Hence, in view of the non-degeneracy assumption on the spectrum, one has $\left\langle i_{0}|A| j_{0}\right\rangle=0$ if $i_{0} \neq j_{0}$, so that $A$ is diagonal in the basis $\{|i\rangle\}$. This basis being arbitrary, one concludes that $A$ is proportional to the identity operator. Thus Eq. (B2) holds true. Thanks to this property and since $D_{k k}$ is self-adjoint and has trace one, Eq. (B1) yields

$$
D_{k k}=\operatorname{Tr}_{B}\left(\left|\Psi_{k}\right\rangle\left\langle\Psi_{k}\right|\right)=n_{A}^{-1} \mathbb{1}_{A} .
$$

Similarly, $\left(D_{k l}+D_{l k}\right) / 2$ and $\left(D_{k l}-D_{l k}\right) / 2$ i are self-adjoint and have vanishing traces for $k \neq l$, so that according to Eqs. (B1) and (B2) one has $D_{k l}=0$ for $k \neq l$. 
One deduces from Eq. (B4) that $\left|\Psi_{k}\right\rangle$ is a maximally entangled pure state, i.e., it has the form given in Eq. (114) (this follows by observing that the Schmidt coefficients of $\left|\Psi_{k}\right\rangle$ are the eigenvalues of the reduced state $D_{k k}$ ). For $k \neq l$, the identity $D_{k l}=0$ is then equivalent to $\left\langle\chi_{l j} \mid \chi_{k i}\right\rangle=0$ for all $i, j=1, \ldots, n_{A}$. As a result, $\varrho=\sum_{k} p_{k}\left|\Psi_{k}\right\rangle\left\langle\Psi_{k}\right|$ is a convex combination of some maximally entangled states $\left|\Psi_{k}\right\rangle$ given by Eq. (114) and satisfying the orthogonality conditions stated after this equation. As emphasized in Section VIIB, any maximally entangled state is given by such a convex combination. We have thus proven that for the Bures and Hellinger distances, $D^{R}(\varrho)=1$ implies that $\varrho$ is a maximally entangled state.

By a similar reasoning, the converse statement is also true provided that the eigenvalues $\lambda_{i} \in \Lambda$ of the family of unitary operators $\mathcal{U}_{\Lambda}$ in the definition of the discord of response satisfy $\sum_{i} \lambda_{i}=0$. This is in particular the case when the $\lambda_{i}$ are the roots of unity, as considered in this paper.

To show that $D^{R}$ satisfies Axiom (v) also for the trace distance, we make use of the trivial bound of Eq. (41). Accordingly, $D_{\operatorname{Tr}}^{R}(\varrho) \leq 1$ for any bipartite state $\varrho$, with equality holding if and only if $D_{\mathrm{Bu}}^{R}(\varrho)=1$. It has been proven above that this is equivalent to $\varrho$ being maximally entangled, hence the result.

Let us finally discuss the case of the Hilbert-Schmidt distance. From Eq. (A1) one gets

$$
D_{\mathrm{HS}}^{R}(\varrho)=\operatorname{Tr} \varrho^{2}-\max _{U_{A} \in \mathcal{U}_{\Lambda}} \operatorname{Tr} \varrho U_{A} \otimes \mathbb{1} \varrho U_{A}^{\dagger} \otimes \mathbb{1} \leq 1 .
$$

This inequality is saturated if and only if the two following conditions are satisfied: (a) $\operatorname{Tr} \varrho^{2}=1$ and (b) $\varrho$ and $U_{A} \otimes \mathbb{1} \varrho U_{A}^{\dagger} \otimes \mathbb{1}$ have orthogonal supports. Thus, by the above arguments, $D_{\mathrm{HS}}^{R}(\varrho)=1$ if and only if $\varrho$ is a maximally entangled pure state. However, if the space dimensions of the two subsystems $A$ and $B$ are such that $n_{B} \geq 2 n_{A}$, one can find maximally entangled states $\varrho$ of $A B$ which are convex combinations of two orthogonal maximally entangled pure states given by Eq. (114). Such states have purities $\operatorname{Tr} \varrho^{2}<1$ and consequently $D_{\mathrm{HS}}^{R}(\varrho)<1$. Therefore, the Hilbert-Schmidt discord of response does not satisfy Axiom (v). The proof of Theorem 13 is now complete.

We have established that the discord of response satisfies Axiom (v) for the Bures, Hellinger, and trace distances. Let us now study whether such Axiom holds true as well for the geometric discord and the measurement-induced geometric discord. For the Bures distance, it is already known that the answer is positive for the geometric discord $D_{\mathrm{Bu}}^{G}$ (see Ref. [9]). Moreover, Theorem 6] above implies that this is also the case for $D_{\mathrm{Bu}}^{M}$. For the Hellinger distance, one finds with the help of the bound $D_{\mathrm{He}}^{R} \leq 1$ and the fundamental relations in Eq. (95) that the geometric discord $D_{\mathrm{He}}^{G}$ is bounded from above by $D_{\max }=2-2 / \sqrt{n_{A}}$ when $n_{A}=2$ and $n_{A}=3$, with equality $D_{\mathrm{He}}^{G}(\varrho)=D_{\max }$ holding if and only if $D_{\mathrm{He}}^{R}(\varrho)=1$. Thanks to Theorem 13, it follows that $D_{\mathrm{He}}^{G}$ satisfies Axiom (v) for $n_{A}=2$ and $n_{A}=3$. We believe but so far have not been able to prove that this remains true for a reference subsystem $A$ with higherdimensional Hilbert spaces, $n_{A}>3$. Whether $D_{\mathrm{He}}^{M}, D_{\mathrm{Tr}}^{G}$, and $D_{\mathrm{Tr}}^{M}$ obey Axiom (v) are other open issues. For the last two measures, we only know so far that the answer is affirmative when $A$ is a qubit, since then $D_{\operatorname{Tr}}^{G}=D_{\operatorname{Tr}}^{M}=D_{\operatorname{Tr}}^{R}$ by

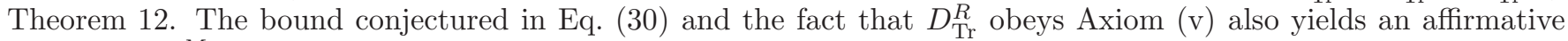
answer for $D_{\mathrm{He}}^{M}$ if both $A$ and $B$ are qubits. All these results are summarized in Table III

\section{Appendix C: Maximally quantum-correlated two-qubit states with a fixed purity}

\section{Two-qubit states with a fixed purity maximizing the trace discord of response}

We derive in this Appendix the maximal values of the trace discord of response $D_{\operatorname{Tr}}^{R}(\varrho)$ for two-qubit states $\varrho$ with a fixed purity $P$, which are given by Eq. (112). Our calculation is based on the following conjecture on the most discordant states for $D_{\operatorname{Tr}}^{R}$ :

Conjecture 2. Among the two-qubit states @ with global state purity $P$, those with maximum trace discord of response are

$$
\varrho_{\max }^{P<3 / 8}=\frac{1}{4}\left((1+\sqrt{8 P-2})\left|\Phi_{+}\right\rangle\left\langle\Phi_{+}|+(1-\sqrt{8 P-2})| \Phi_{-}\right\rangle\left\langle\Phi_{-}|+| \Psi_{+}\right\rangle\left\langle\Psi_{+}|+| \Psi_{-}\right\rangle\left\langle\Psi_{-}\right|\right)
$$

for $\frac{1}{4} \leq P \leq \frac{3}{8}$, and

$$
\varrho_{\max }^{P>3 / 8}=\frac{1}{6}\left((2-\sqrt{6 P-2})\left(\left|\Phi_{+}\right\rangle\left\langle\Phi_{+}|+| \Phi_{-}\right\rangle\left\langle\Phi_{-}\right|\right)+(2+2 \sqrt{6 P-2})\left|\Psi_{+}\right\rangle\left\langle\Psi_{+}\right|\right)
$$

for $\frac{3}{8}\left\langle P \leq 1\right.$. Here, $\left.\mid \Psi_{ \pm}\right\rangle=(|01\rangle \pm|10\rangle) / \sqrt{2}$ and $\left|\Phi_{ \pm}\right\rangle=(|00\rangle \pm|11\rangle) / \sqrt{2}$ refer to the four Bell states.

This conjecture relies on a thorough numerical analysis using randomly generated states, as described in Sec. VII. In what follows we determine the values of $D_{\operatorname{Tr}}^{R}\left(\varrho_{\max }^{P}\right)$ as a function of $P$. Since $A$ is a qubit, the unitaries $U_{A}$ acting on $\mathcal{H}_{A} \simeq \mathbb{C}^{2}$ with spectrum $\Lambda=\{-1,1\}$ can be decomposed in terms of the three Pauli matrices $\sigma_{x}, \sigma_{y}$, and $\sigma_{z}$ as

$$
U_{A}=\sin \theta \cos \phi \sigma_{x}+\sin \theta \sin \phi \sigma_{y}+\cos \theta \sigma_{z},
$$


with some arbitrary angles $\theta \in[0, \pi]$ and $\phi \in\left[0,2 \pi\left[\right.\right.$. We will show that the trace distance between $\varrho_{\text {max }}^{P}$ and the unitarily perturbed state $U_{A} \otimes \mathbb{1} \varrho_{\max }^{P} U_{A}^{\dagger} \otimes \mathbb{1}$ does not depend on $U_{A}$. Recall that $d_{\operatorname{Tr}}(\varrho, \sigma)$ is equal to the sum of the moduli of the eigenvalues of $\varrho-\sigma$. For the density matrix $\varrho$ max $2<3$ given by Eq. (C1), the corresponding eigenvalues are $\pm \frac{1}{4} \sqrt{8 P-2}(1+\cos \theta)$ and $\pm \frac{1}{4} \sqrt{8 P-2}(1-\cos \theta)$. The sum of their moduli does not depend on $(\theta, \phi)$, that is, this sum is independent of $U_{A}$. The maximum trace discord of response for states with purity $P \leq 3 / 8$ reads

$$
D_{\operatorname{Tr}}^{R}\left(\varrho_{\max }^{P<3 / 8}\right)=\frac{1}{4}\left\|\varrho_{\max }^{P<3 / 8}-U_{A} \otimes \mathbb{1} \varrho_{\max }^{P<3 / 8} U_{A}^{\dagger} \otimes \mathbb{1}\right\|_{\operatorname{Tr}}^{2}=2 P-\frac{1}{2} .
$$

For the state $\varrho_{\max }^{P>3 / 8}$ given by Eq. (C2), the corresponding eigenvalues are $\pm \frac{1}{4}(2-\beta+f(\beta, \theta))$ and $\pm \frac{1}{4}(2-\beta-f(\beta, \theta))$, where we have set $\beta=\frac{2}{3}(2-\sqrt{6 P-2})$ and

$$
f(\beta, \theta)=\frac{1}{\sqrt{2}} \sqrt{8+\beta^{2}(5-3 \cos (2 \theta))-4 \beta(3-\cos (2 \theta))} .
$$

Once again, the sum of the moduli of these eigenvalues does not depend on the angles $\theta$ and $\phi$ of the unitary matrix. Therefore, the corresponding maximum trace discord of response reads

$$
D_{\operatorname{Tr}}^{R}\left(\varrho_{\max }^{P>3 / 8}\right)=\frac{1}{9}(1+\sqrt{6 P-2})^{2} .
$$

\section{Two-qubit states with a fixed purity maximizing the Hellinger discord of response}

We now study the same problem as before but for the Hellinger discord of response $D_{\mathrm{He}}^{R}$. Let us remark that for two-qubit states the analytical expression of $D_{\mathrm{He}}^{R}(\varrho)$ is given by Eq. (22). The maximally quantum-correlated states according to $D_{\mathrm{He}}^{R}$ are found by a thorough numerical investigation, as described in Section VII In the range of values $1 / 4 \leq P \leq 1 / 3$ of the global state purity $P$, these states are the Werner states defined in Eq. (113) with parameter $1 \leq a \leq 4 / 3$. Note that such Werner states also maximize the Hilbert-Schmidt discord of response $D_{\mathrm{HS}}^{R}$. Hence in the range $1 / 4 \leq P \leq 1 / 3$, corresponding to region $\mathrm{I}$ in Fig. 目(c), the maximal Hellinger discord of response reads

$$
D_{\mathrm{He}}^{R}\left(\varrho_{W}\right)=\frac{1}{6}(-\sqrt{12 \mathrm{P}-3}-\sqrt{6} \sqrt{-6 \mathrm{P}-\sqrt{12 \mathrm{P}-3}+3}+3) .
$$

In the range $1 / 3 \leq P \leq 0.503$, corresponding to region II in Fig. 4(c), we find numerically that the maximally quantum-correlated states with respect to $D_{\mathrm{He}}^{R}$ belong to the following family of matrices of rank 3 :

$$
\varrho_{\max }^{1 / 3 \leq P \leq 0.503}=\left(\begin{array}{cccc}
\frac{1}{2}+a-b & 0 & 0 & 0 \\
0 & 2 b \cos ^{2} \phi & 2 b \cos \phi \sin \phi & 0 \\
0 & 2 b \cos \phi \sin \phi & 2 b \sin ^{2} \phi & 0 \\
0 & 0 & 0 & \frac{1}{2}-a-b
\end{array}\right),
$$

where the condition of fixed purity yields $a=\frac{1}{2} \sqrt{-12 b^{2}+4 b+2 P-1}$. The optimal values of $b$ and $\phi$ are found by numerical maximization of $D_{\mathrm{He}}^{R}$ for these states.

For global state purities $P>0.503$, corresponding to region III in Fig. 4(c), we find the following maximally quantum-correlated states of rank 2 :

$$
\varrho_{\max }^{P>0.503}=\left(\begin{array}{cccc}
1-2 b & 0 & 0 & 0 \\
0 & 2 b \cos ^{2} \phi & 2 b \cos \phi \sin \phi & 0 \\
0 & 2 b \cos \phi \sin \phi & 2 b \sin ^{2} \phi & 0 \\
0 & 0 & 0 & 0
\end{array}\right)
$$

The condition of fixed purity enable us to eliminate one variable: $b=\frac{1}{4}(\sqrt{2 P-1}+1)$. For a given purity $P$, the parameter $\phi$ for which $D_{\mathrm{He}}^{R}$ achieves its maximum reads

$\cos \phi=\frac{-1}{2 \sqrt{2-2 P}}\left(-4 P-\sqrt{2-2 P}+\sqrt{-4 P^{2}+6 P-2}+4+2 \sqrt{(P-1)\left(3 P-2 \sqrt{2-2 P}-3 \sqrt{2 P-1}+2 \sqrt{-4 P^{2}+6 P-2}\right)}\right)^{1 / 2}$.

The corresponding maximum Hellinger discord of response as a function of $P$, determined with the help of Eq. (22), is shown in Fig. $4(\mathrm{c})$. 


\section{Appendix D: Hilbert-Schmidt geometric discord for qubit-qudit states with maximally mixed marginals}

In this Appendix we show that the Hilbert-Schmidt geometric discord is equal to its lower bound in Eq. (119) if the subsystem $A$ is a qubit and the state $\varrho$ has maximally mixed marginals. Although $D_{\mathrm{HS}}^{G}$ is not a proper measure of quantum correlations, it can play a useful role since it provides bounds on the other geometric measures (e.g., the trace geometric discord satisfies $2 D_{\mathrm{HS}}^{G}(\varrho) \leq D_{\mathrm{Tr}}^{G}(\varrho) \leq 2 n_{B} D_{\mathrm{HS}}^{G}(\varrho)$, see Theorem 12 and Eqs. (15) and (A5)). Moreover, $D_{\text {HS }}^{G}$ gives the value of the Hellinger geometric discord by taking the square root of the state $\varrho$ (see Theorem 2 ).

Proposition 1. Let $A$ be a qubit and $B$ a qudit with Hilbert space of arbitrary finite dimension $n_{B}$. If the global state $\varrho$ of $A B$ has maximally mixed marginals $\varrho_{A}=\mathbb{1}_{A} / 2$ and $\varrho_{B}=\mathbb{1}_{B} / n_{B}$, then the Hilbert-Schmidt geometric discord of $\varrho$ is equal to the sum of the two smallest squared singular values $\mu_{3}$ and $\mu_{4}$ of the reshuffled density matrix $\varrho^{\mathcal{R}}$ :

$$
D_{\mathrm{HS}}^{G}(\varrho)=\mu_{3}+\mu_{4} .
$$

This Proposition enable us to calculate quite easily the Hilbert-Schmidt geometric discord for a wide class of qubitqudit states with maximally mixed partial states, such as the states known from the theory of quantum channels as the renormalized dynamical matrices of bistochastic CPTP maps [37]. The advantage of expressing the geometric discord in terms of the singular values of $\varrho^{\mathcal{R}}$ is that we can do that in an arbitrary basis. As other examples of states which satisfy the conditions of the Proposition, let us mention the Werner-like rotationally invariant states defined and analyzed in Ref. [77] (see also Ref. [78]) and the qubit-qudit states given by the r.h.s. of Eq. (18) with $\vec{x}=\vec{y}=\overrightarrow{0}$, $t_{0}=1 / \sqrt{2 n_{B}}$, and $t_{m p} \geq 0, \sum_{m p} t_{m p}=\sqrt{2 n_{B}}$.

The proof of Proposition 1 relies on the following result on the Hilbert-Schmidt geometric discord for qubit-qudit systems (see e.g. Ref. 24], Theorem 2). An arbitrary qubit-qudit density matrix $\varrho$ can always be written in the so-called Fano form, namely:

$$
\varrho=\sum_{m=0}^{3} \sum_{p=0}^{n_{B}^{2}-1} M_{m p} \hat{\sigma}_{m} \otimes \hat{\gamma}_{p}
$$

where $\hat{\sigma}_{0} \equiv \mathbb{1}_{A} / \sqrt{2}, \hat{\sigma}_{1}, \hat{\sigma}_{2}$, and $\hat{\sigma}_{3}$ are the Pauli matrices renormalized in such a way $\operatorname{that} \operatorname{Tr}\left(\hat{\sigma}_{m} \hat{\sigma}_{n}\right)=\delta_{m n}$, and $\hat{\gamma}_{p}$ are Hermitian matrices forming an orthonormal basis for the space of $n_{B} \times n_{B}$ matrices $\left(\right.$ i.e., $\left.\operatorname{Tr}\left(\hat{\gamma}_{p} \hat{\gamma}_{q}\right)=\delta_{p q}\right)$. The components $M_{m p}$ of $\varrho$ in Eq. (D2) are given by $M_{m p}=\operatorname{Tr}\left(\varrho \hat{\sigma}_{m} \otimes \hat{\gamma}_{p}\right)$ and form a $4 \times n_{B}^{2}$ real matrix $M$ (covariance matrix). Define the $3 \times n_{B}^{2}$ matrix $\widetilde{M}$ obtained from $M$ by removing the first row, that is, $\widetilde{M}=$ $\left[M_{m p}\right]_{m=1, \ldots, 3, p=0, \ldots, n_{B}^{2}-1}$. It can be shown that $D_{\mathrm{HS}}^{G}(\varrho)$ is equal to the sum of the two smallest squared singular values of $\widetilde{M}$, that is,

$$
D_{\mathrm{HS}}^{G}(\varrho)=\operatorname{sv}_{2}^{2}(\widetilde{M})+\operatorname{sv}_{3}^{2}(\widetilde{M})
$$

where $\operatorname{sv}_{1}^{2}(\widetilde{M}) \geq \operatorname{sv}_{2}^{2}(\widetilde{M}) \geq \operatorname{sv}_{3}^{2}(\widetilde{M})$ denote the eigenvalues of the $3 \times 3$ non-negative matrix $\widetilde{M}(\widetilde{M})^{\dagger}$

Before proceeding to the proof of Proposition 1 let us start with some technical considerations on vectorization. This operation transforms a $n \times n$ matrix $Y$ into the vector $|Y\rangle$ obtained by ordering the matrix entries into a one-column vector, that is, $|Y\rangle$ has $n^{2}$ components given by

$$
\langle i, j \mid Y\rangle \equiv\langle i|Y| j\rangle \equiv\langle\bar{Y} \mid i, j\rangle
$$

where the bar denotes complex conjugation in the standard basis $\{|i\rangle\}$. Let $X, Y$, and $Z$ be matrices of sizes $n_{A} n_{B} \times n_{A} n_{B}, n_{A} \times n_{A}$, and $n_{B} \times n_{B}$, respectively. The following chain of identities will be useful:

$$
\operatorname{Tr} X(Y \otimes Z)=\sum_{i j k l}\langle i, k|X| j, l\rangle\langle j|Y| i\rangle\langle l|Z| k\rangle=\sum_{i j k l}\left\langle i, j\left|X^{\mathcal{R}}\right| k, l\right\rangle\left\langle Y^{\dagger} \mid i, j\right\rangle\left\langle k, l \mid Z^{T}\right\rangle=\left\langle Y^{\dagger}\left|X^{\mathcal{R}}\right| Z^{T}\right\rangle,
$$

where $\mathcal{R}$ is the reshuffling operation defined in Eq. (117) and $T$ denotes the transposition in the standard basis. Now let us introduce the orthonormal bases $\left\{\left|\sigma_{m}\right\rangle\right\}_{m=0}^{3}$ of $\mathbb{C}^{4}$ and $\left\{\left|\gamma_{p}\right\rangle\right\}_{p=0}^{n_{B}^{2}-1}$ of $\mathbb{C}^{n_{B}^{2}}$ obtained from the vectorization of the matrices $\hat{\sigma}_{m}$ and $\hat{\gamma}_{p}$ appearing in the decomposition of Eq. (D2). On account of Eq. (D5), the matrix $M$ in this decomposition coincides with the reshuffled density matrix $\varrho^{\mathcal{R}}$ in the vectorized bases $\left\{\left|\sigma_{m}\right\rangle\right\}_{m=0}^{3}$ and $\left\{\left|\overline{\gamma_{p}}\right\rangle\right\}_{p=0}^{n_{B}^{2}-1}$ :

$$
M_{m p}=\operatorname{Tr}\left(\varrho \hat{\sigma}_{m} \otimes \hat{\gamma}_{p}\right)=\left\langle\sigma_{m}\left|\varrho^{\mathcal{R}}\right| \overline{\gamma_{p}}\right\rangle
$$

The proof of Proposition 1 uses the following lemma.

Lemma 1. Let $\varrho$ be a state of $A B$ satisfying the assumptions of Proposition 1 . Then the largest singular value of $\varrho^{\mathcal{R}}$ is equal to $1 / \sqrt{2 n_{B}}$. 
Proof. We use the formal correspondence (via the Jamiołkowski isomorphism, see e.g. Ref. [37]) of the states with maximally mixed marginals with quantum channels (i.e., CPTP maps) that preserve the maximally mixed state. Let us introduce the $n_{A} \times n_{B}$ matrices $K_{\alpha}$ with matrix elements

$$
\left\langle i_{A}\left|K_{\alpha}\right| k_{B}\right\rangle \equiv \sqrt{p_{\alpha}}\left\langle i_{A}, k_{B} \mid \Psi_{\alpha}\right\rangle
$$

where $p_{\alpha}$ and $\left|\Psi_{\alpha}\right\rangle$ are, respectively, the eigenvalues and eigenvectors of $\varrho$. From Eqs. (117) and (D4) it can be immediately derived that

$$
\varrho^{\mathcal{R}}=\sum_{\alpha} K_{\alpha} \otimes \overline{K_{\alpha}} \quad, \quad\left(\varrho^{\mathcal{R}}\right)^{\dagger}=\sum_{\alpha} K_{\alpha}^{\dagger} \otimes \overline{K_{\alpha}^{\dagger}}
$$

and

$$
\operatorname{Tr}_{A}(\varrho)=\sum_{\alpha} \overline{K_{\alpha}^{\dagger} K_{\alpha}}=\frac{1}{n_{B}} \mathbb{1}_{B} \quad, \quad \operatorname{Tr}_{B}(\varrho)=\sum_{\alpha} K_{\alpha} K_{\alpha}^{\dagger}=\frac{1}{2} \mathbb{1}_{A}
$$

where the bar denotes the complex conjugation in the standard basis, i.e., $\left\langle i_{A}\left|\overline{K_{\alpha}}\right| k_{B}\right\rangle=\left\langle i_{A}\left|K_{\alpha}\right| k_{B}\right\rangle^{*}$. The above relations show that the operators $n_{B} K_{\alpha}^{\dagger} K_{\alpha}$ satisfy the completeness condition $\sum_{\alpha} n_{B} K_{\alpha}^{\dagger} K_{\alpha}=\mathbb{1}_{B}$. Therefore, $\sqrt{n_{B}} K_{\alpha}$ can be interpreted as the Kraus operators of some quantum operation. Also, the formula given in Eq. (D8) is proportional to the superoperator form of a quantum operation, as defined by Eq. (116). The same considerations apply to $\left(\varrho^{\mathcal{R}}\right)^{\dagger}$, which is proportional to the superoperator form of the quantum operation defined by the Kraus operators $L_{\alpha} \equiv \sqrt{2} K_{\alpha}^{\dagger}$. Since the composition of two quantum operations is still a quantum operation $[79], 2 n_{B} \varrho^{\mathcal{R}}\left(\varrho^{\mathcal{R}}\right)^{\dagger}$ is thus a CPTP map (in its superoperator form), which moreover preserves the identity. From the quantum analogue of the Frobenius-Perron theorem [80], one concludes that the leading eigenvalue of such a map is equal to one (see also Theorem 1 of Ref. [81]). Therefore, the largest singular value of $\varrho^{\mathcal{R}}$ is equal to $1 / \sqrt{2 n_{B}}$.

Proof of Proposition 1 . Since the partial traces of $\varrho$ are by assumption proportional to $\mathbb{1}$, we have Tr $\varrho \hat{\sigma}_{m} \otimes \mathbb{1}_{B}=$ $\operatorname{Tr} \hat{\sigma}_{m} / 2=0$ for $m=1, \ldots, 3$, and similarly $\operatorname{Tr} \varrho \mathbb{1}_{A} \otimes \overline{\hat{\gamma_{p}}}=\overline{\operatorname{Tr} \hat{\gamma_{p}}} / n_{B}=0$ for $p=1, \ldots, n_{B}^{2}-1$. Hence, in the first row and first column of the matrix $M$, only one entry is different from 0 , namely $M_{00}=\operatorname{Tr} \varrho \mathbb{1}_{A} \otimes \mathbb{1}_{B} / \sqrt{2 n_{B}}=1 / \sqrt{2 n_{B}}$, which is therefore a singular value of $M$. Analogously, the matrix $\widetilde{M}$ has only zeros in its first column. Thus $M$ and $\widetilde{M}$ have the same singular values, excepted for the additional singular value $\mu_{1}=1 / \sqrt{2 n_{B}}$ of $M_{\text {. According to }}$ Eq. (D6) and Lemma 1. $\varrho^{\mathcal{R}}$ has the same singular values as $M$ and its largest singular value is $\mu_{1}$. It thus follows from Eq. (D3) that $D_{\mathrm{HS}}^{G}(\varrho)$ is the sum of the two smallest squared singular values of $\varrho^{\mathcal{R}}$.

[1] W. H. Zurek, Annalen der Physik 9, 855 (2000).

[2] H. Ollivier and W. H. Zurek, Phys. Rev. Lett. 88, 017901 (2001).

[3] L. Henderson and V. Vedral, J. Phys. A: Math. Gen. 34, 6899 (2001).

[4] D. Girolami and G. Adesso, Phys. Rev. A 83, 052108 (2011).

[5] K. Modi, A. Brodutch, H. Cable, T. Paterek, and V. Vedral, Rev. Mod. Phys. 84, 1655 (2012).

[6] Y. Huang, New J. Phys. 16, 033027 (2014).

[7] B. Dakić, V. Vedral, and Č. Brukner, Phys. Rev. Lett. 105, 190502 (2010).

[8] B. Aaronson, R. Lo Franco, and G. Adesso, Phys. Rev. A 88, 012120 (2013).

[9] D. Spehner and M. Orszag, New J. Phys. 15, 103001 (2013).

[10] D. Spehner and M. Orszag, J. Phys. A: Math. Theor. 47, 035302 (2014).

[11] W. Roga, S. M. Giampaolo, and F. Illuminati, J. Phys A: Math. Theor. 47, 365301 (2014).

[12] K. Modi, T. Paterek, W. Son, V. Vedral, and M. Williamson, Phys. Rev. Lett. 104, 080501 (2010).

[13] S. M. Giampaolo, A. Streltsov, W. Roga, D. Bruß, and F. Illuminati, Phys. Rev. A 87, 012313 (2013).

[14] F. Ciccarello, T. Tufarelli, and V. Giovannetti, New J. Phys. 16, 013038 (2014).

[15] D. Spehner, J. Math. Phys. 55, 075211 (2014).

[16] M. Piani, V. Narasimhachar, and J. Calsamiglia, New J. Phys. 16, 113001 (2014)

[17] L. Chang and S. Luo, Phys. Rev. A 87, 062303 (2013).

[18] A. Uhlmann, Rep. Math. Phys. 9, 273 (1976).

[19] D. Bures, Trans. Am. Math. Soc. 135, 199 (1969).

[20] S. Luo and S. Fu, Phys. Rev. A 82, 034302 (2010).

[21] T. Nakano, M. Piani, and G. Adesso, Phys. Rev. A 88, 012117 (2013).

[22] A. Streltsov, H. Kampermann, and D. Bruß, Phys. Rev. Lett. 107, 170502 (2011).

[23] T. Abad, V. Karimipour, and L. Memarzadeh, Phys. Rev. A 86, 062316 (2012).

[24] S. Gharibian, Phys. Rev. A 86, 042106 (2012).

[25] K.P. Seshadreesan and M.M. Wilde, arXiv:1410.1441 [quant-ph] 
[26] S. Pirandola, C. Lupo, V. Giovannetti, S. Mancini, and S. L. Braunstein, New J. Phys. 13, 113012 (2011).

[27] S.-H. Tan, B. I. Erkmen, V. Giovannetti, S. Guha, S. Lloyd, L. Maccone, S. Pirandola, and J.H. Shapiro, Phys. Rev. Lett. 101, $253601(2008)$

[28] A. Farace, A. De Pasquale, L. Rigovacca, and V. Giovanetti, New J. Phys. 16, 073010 (2014).

[29] D. Girolami, A. M. Souza, V. Giovannetti, T. Tufarelli, J. G. Filgueiras, R. S. Sarthour, D. O. Soares-Pinto, I. S. Oliveira, and G. Adesso, Phys. Rev. Lett. 112, 210401 (2014).

[30] L. A. Correa, J. P. Palao, D. Alonso, and G. Adesso, Sci. Rep. 4, 3949 (2014).

[31] D. Girolami, T. Tufarelli, and G. Adesso, Phys. Rev. Lett. 110, 240402 (2013).

[32] W. Roga, D. Buono, and F. Illuminati, New J. Phys. 17, 013031 (2015).

[33] A. Streltsov, G. Adesso, M. Piani, and D. Bruß, Phys. Rev. Lett. 109, 050503 (2012).

[34] M. Piani, Phys. Rev. A 86, 034101 (2012).

[35] D. Perez-Garcia, M. M. Wolf, D. Petz, and M. B. Ruskai, J. Math. Phys. 47, 083506 (2006).

[36] M. Ozawa, Phys. Lett. A 268, 158 (2000).

[37] I. Bengtsson and K. Życzkowski, Geometry of Quantum States: An Introduction to Quantum Entanglement, Cambridge University Press, Cambridge (UK), 2006.

[38] C. H. Bennett, D. P. DiVincenzo, J. A. Smolin, and W. K. Wootters, Phys. Rev. A 54, 3824 (1996).

[39] A. Datta, A. Shaji, and C. M. Caves, Phys. Rev. Lett. 100, 050502 (2008).

[40] E. P. Wigner and M. M. Yanase, Proc. Natl. Acad. Sci. U.S.A. 49, 910 (1963).

[41] S. Luo, Phys. Rev. Lett. 91, 180403 (2003).

[42] K. Audenaert, L. Cai, and F. Hansen, Lett. Math. Phys. 85, 135 (2008).

[43] M. A. Nielsen and I. L. Chuang, Quantum Computation and Information, Cambridge University Press, Cambridge (UK), 2000.

[44] D. Petz, Lin. Alg. and its Appl. 244, 81 (1996).

[45] C. A. Fuchs and J. van de Graaf, IEEE Trans. Inf. Theory 45, 1216 (1999).

[46] K. M. R. Audenaert, J. Calsamiglia, R. Munoz-Tapia, E. Bagan, L. Masanes, A. Acin, and F. Verstraete, Phys. Rev. Lett. 98, 160501 (2007).

[47] E. H. Lieb, Adv. Math. 11, 267 (1973).

[48] A. S. Holevo, Theor. Math. Phys. 13, 184 (1972).

[49] A. Monras, G. Adesso, S. M. Giampaolo, G. Gualdi, G. B. Davies, and F. Illuminati, Phys. Rev. A 84, 012301 (2011).

[50] S. M. Giampaolo and F. Illuminati, Phys. Rev. A 76, 042301 (2007).

[51] T. C. Wei and P. M. Goldbart, Phys. Rev. A 68, 042307 (2003).

[52] M. Blasone, F. Dell'Anno, S. De Siena, and F. Illuminati, Phys. Rev. A 77, 062304 (2008).

[53] A. Streltsov, H. Kampermann, and D. Bruß, New J. Phys. 12, 123004 (2010).

[54] G. Vidal, J. Mod. Opt. 47, 355 (2000).

[55] C. W. Helstrom, Quantum Detection and Estimation Theory, Academic Press, New York (US), 1976.

[56] F. M. Paula, T. R. de Oliveira, and M. S. Sarandy, Phys. Rev. A 87, 064101 (2013).

[57] P. Marian and T. A. Marian, J. Phys. A: Math. Theor. 48, 115301 (2015).

[58] D. Buono, W. Roga, and F. Illuminati, "Quantum correlations of Gaussian states: characterization, quantification, and comparison by metrics, states, and operations", to appear (2015).

[59] V. Vedral and M. B. Plenio, Phys. Rev. A 57, 1619 (1998).

[60] S. Luo and S. Fu, Theor. Math. Phys. 171, 870 (2012).

[61] H. Barnum and E. Knill, J. Math. Phys. 43, 2097 (2002).

[62] M. B. Plenio, Phys. Rev. Lett. 95, 090503 (2005).

[63] E. A. Carlen, Trace inequalities and quantum entropy: An introductory course, in: Entropy and the quantum, R. Sims and D. Ueltschi (eds.), Contemp. Math. 529, 73 (Amer. Math. Soc., Providence, RI, 2010).

[64] R. Bhatia, Matrix Analysis, Springer (1991).

[65] T. Ogawa and H. Nagaoka, IEEE Trans. Inf. Theory 45, 2486 (1999).

[66] J. Tyson, J. Math. Phys. 50, 032106 (2009).

[67] R. Bhatia and F. Kittaneh, Lin. Alg. and its Appl. 318, 109 (2000)

[68] J. Korbicz, P. Horodecki, and R. Horodecki, Phys. Rev. A 86, 042319 (2012).

[69] S. Rana and P. Parashar, Phys. Rev. A 85, 024102 (2012)

[70] K. Chen and L.-A. Wu, Quant. Inf. Comp. 3, 193 (2003).

[71] R. Horn and C. R. Johnson, Matrix analysis, Cambridge University Press, 1985 (22nd printing 2009), page 449, Example 7.4.52.

[72] S. Pirandola, Phys. Rev. Lett. 106, 090504 (2011)

[73] G. Adesso, Phys. Rev. A 90, 022321 (2014).

[74] G. Adesso, V. D’Ambrosio, E. Nagali, M. Piani, and F. Sciarrino, Phys. Rev. Lett. 112, 140501 (2014).

[75] T.-C. Wei, K. Nemoto, P. M. Goldbart, P. G. Kwiat, W. J. Munro, and F. Verstraete, Phys. Rev. A 67, 022110 (2003).

[76] G. Adesso and F. Illuminati, Phys. Rev. A 72, 032334 (2005).

[77] D. Chruścinski and A. Kossakowski, Open Sys. Inf. Dyn. 14, 25 (2007).

[78] H.-P. Breuer, J. Phys. A: Math. Gen. 38, 9019 (2005).

[79] W. Roga, M. Fannes, and K. Życzkowski, J. Phys. A: Math. Theor. 41, 035305 (2008).

[80] W. Bruzda, V. Cappellini, H.-J. Sommers, and K. Życzkowski, Phys. Lett. A 373, 320 (2009).

[81] W. Roga, Z. Puchała, Ł. Rudnicki, and K. Życzkowski, Phys. Rev. A 87, 032308 (2013). 